\title{
Revisions of Australian ground-hunting spiders: I. Amauropelma gen. nov. (Araneomorphae: Ctenidae)
}

\author{
Robert J. Raven ${ }^{1}$, Kylie Stumkat ${ }^{1}$ and Michael R. Gray ${ }^{2}$ \\ ${ }^{1}$ Queensland Museum, PO Box 3300, South Brisbane, Queensland 4101, Australia \\ ${ }^{2}$ Australian Museum, College St, Sydney South, New South Wales 2000, Australia
}

\begin{abstract}
Revisions of Australian ground-hunting spiders: I. Amauropelma gen. nov. (Aranemorphae: Ctenidae). A new genus Amauropelma is described from north-eastern Australia and includes 16 new species: Amauropelma anzses, Amauropelma bluewater, Amauropelma claudie, Amauropelma gayundah, Amauropelma gordon, Amauropelma hasenpuschi, Amauropelma leo, Amauropeima mcilwraith, Amauropelma monteithi, Amauropelma mossman, Amauropelma pineck, Amauropelma rifleck, Amauropelma torbjorni, Amauropelma trueloves (type species), Amauropelma undara, and Amauropelma wallaman. The characters of the genus challenge existing family diagnoses of the Miturgidae and Ctenidae.
\end{abstract}

\section{INTRODUCTION}

Araneomorph spider (and to a lesser extent mygalomorph) systematics are currently in a severe state of flux. Platnick, Ovtsharenko and Catley (e.g. Platnick, 2000) are revising the diverse Australian Gnaphosoidea, Diana Silva is revising the Ctenidae, Griswold and others are examining relationships of the Lycosoidea, and Raven and Stumkat are revising the Australian Clubionidae, Miturgidae, Ctenidae, and Zoridae. Family boundaries are changing but stability is coming. The genus described here is one such example. The ambiguity of its placement was nominally resolved only when Dr Diana Silva, American Museum of Natural History, on examining the genus, unequivocally declared it to be a ctenid.

Ctenids are cursorial spiders with two claws, claw tufts, and two recurved eye rows which when viewed from the front it can be seen that the ALE are set high near posterior eyes (e.g., DippenaarSchoeman and Jocqué, 1997). They are typically tropical spiders of the rainforest, heathland, open forest and desert. In most collections, they are still quite rare. The Australian Ctenidae at present include Ctenus agroecoides (Thorell, 1881) from northern Queensland, the blind Janusia muiri Gray, 1973 from caves in Western Australia, and Leptoctenus agalenoides L. Koch, 1878 from Rockhampton, Mackay, mid-eastern Queensland. No members of the family were listed by Rainbow (1911). The spiders described here are small and hunt freely in the rainforest litter of north-eastern Queensland. They have not been found associated with webs or carrying an egg sac. Reports on surveys of the fauna of north-eastern Queensland placed them either in the Miturgidae or Ctenidae
(Davies, 1976, 1977), depending on the anterior eye row curvature. When those taxa are examined together it is clear that the eye differences in this group are interspecific. Hence, the diagnosis of the Ctenidae is challenged and the boundary with the Miturgidae is diffused.

The concept of the Miturgidae has been ambiguous, partially addressed but not resolved by Griswold (1991, 1993, 1994). The family (sensu Lehtinen, 1967 and Griswold, 1993, 1993) included claw-tufted spiders with two recurved eye rows (e.g. Diaprograpta Simon, 1909), although in most genera the back eye row is straight or slightly procurved. The boundary with the Ctenidae seemed clear: ctenids have the smaller ALE set high above AME, near the PLE, forming an eye formula of 2.4.2. (The zorid genus Elassoctenus has ALE set higher but they are closer to the PME than the PLE and do not form the 2.4.2 formula.) The inclusion then of some undescribed species of Amauropelma gen. nov. in the Miturgidae and others in the Ctenidae (e.g. Davies, 1976, 1977) indicates that the eyes of different species fit different families.

The species described here are unequivocally one genus. Males lack tibial cracks and cymbial scopula and the tapetum is grate-shaped. Hence, the genus cladistically rises above the Tengellidae and Zoropsidae (as grouped by Griswold, 1993). Females have lateral teeth on the epigyne and, along with the curvature of the eye rows, the Ctenidae are clearly their closest relatives although cladistically Amauropelma may be the sister group of most other ctenids.

This is the first of a series of papers revising the family in Australia. 


\section{MATERIALS AND METHODS}

All material of Amauropelma was collected in north-eastern Queensland. The left palp is described with the ventral face dorsal. Eye group size is measured through the centre of the group and compared to the carapace width on that line. Abbreviations and methods are standard for the Araneae and explained in Raven (1994). Hair terminology (plumose, feathery) follows Lehtinen (1967). Spination on metatarsi III and IV test the repeatability of spine counts because the boundary of lateral versus ventral spines is often vague. As before (e.g. Raven, 1994), a spine is considered lateral if the base is visible when viewed from above. Measurements, if not otherwise indicated, are in millimetres. Colour is taken from alcoholpreserved specimens unless noted. The median apophysis is cup- or ladle-shaped. The prolateral portion is termed the handle (of the ladle) and is roughly taken from the edge of the lumen. Often the lumen is not visible in ventral view but only from distal axial view or lateral view. The epigyne consists of a plate with two lateral lobes, here termed 'wings', and the lateral teeth are also described as 'horns'. The dimensions of the epigyne are taken from the widest points and length is measured from the plates, not including lateral teeth, and not from the central dimension (see Figure 14g).

Authorship. Statements concerning relationships are those solely of the senior author.

Acronyms: AMNH, American Museum of Natural History, New York; AMS, Australian Museum, Sydney; ANIC, Australian National Insect Collection, Canberra; ANZSES, Australian New Zealand Scientific Exploration Society; QM, Queensland Museum, Brisbane; SAM, South Australian Museum, Adelaide; WAM, Western Australian Museum, Perth.

\section{SYSTEMATICS}

\section{Family Ctenidae}

\section{Amauropelma gen. nov.}

\section{Type Species}

Amauropelma trueloves sp. nov.

\section{Diagnosis}

Ecribellate lycosoid with two claws, welldeveloped claw tufts, eight eyes in two clearly recurved rows, or blind, eyes of similar size, ALE sometimes set back near PLE, tapetum (of PME) grate-shaped; lateral spinnerets all short, conical, with short apical segment; ALS clearly separated; colulus absent; PLS of females cylindrical; tarsal organ (or rod) on legs III, IV set at basal 1/8-1/6;

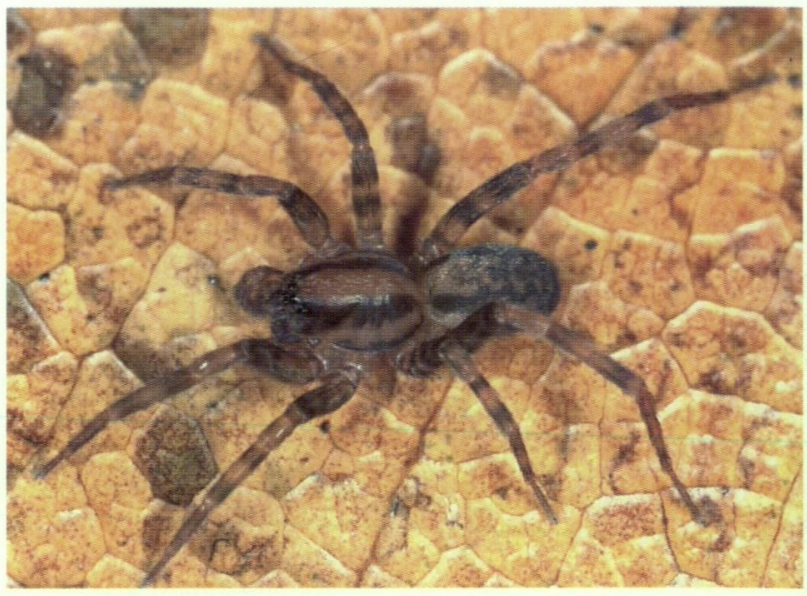

Figure 1 Amauropelma trueloves sp. nov., female, habitus.

more distal on legs I, II. Tibiae I, II with 4-5 pairs of strong spines ventrally; 3 strong pairs ventrally on metatarsi I, II; trochanters deeply notched. Retrocoxal hymen present on leg I; pre-distal tarsal fracture absent. Tarsi with adpressed trichobothria (Figures 10b, 16a). Males without tibial crack on legs; processes retrolaterally on distal patella and tibia of palp; cymbium apically coniform, without scopula dorsally; retrobasal and probasal processes on cymbium of most species; bulb with subtegulartegular interlocking lobes; tegulum large with cupshaped median apophysis, hyaline (filmy) conductor; embolus a large hook-shaped plate, directed clockwise, with 2-4 deep ventral ridges narrowing distally; sperm duct opens on back of plate. Epigyne a plate with soft lateral teeth arising

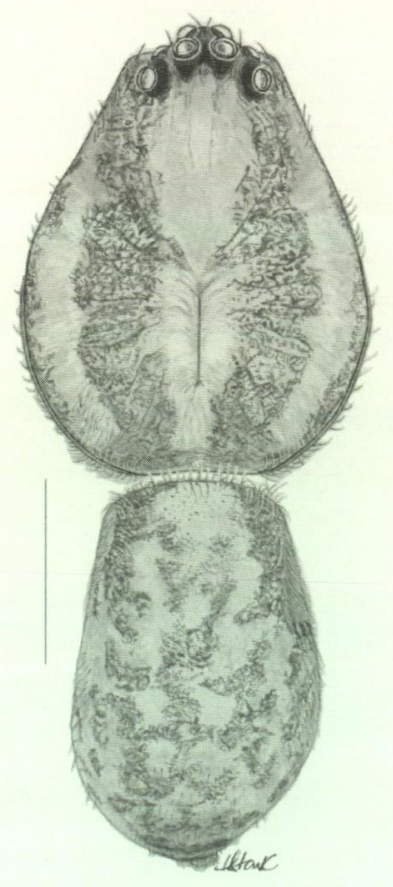

Figure 2 Amauropelma mossman sp. nov., male, carapace and abdomen, dorsal view. Scale line, $1 \mathrm{~mm}$. 
from its dorsal surface; spermathecae simple. Tracheal spiracle near spinnerets, indistinct.

\section{Description}

Cephalothorax pear-shaped; caput broad, low; uniform cover of fine hairs, none feathery or plumose; short fovea. Blind or eight small, similarly-sized eyes, sessile or normal on raised black tubercle in two very recurved rows; rows are 2.2.2.2, 2.4.2 or 4.4. Median eyes set close together, ca. 1 diameter apart; MOQ longer than wide; group narrower in front than behind, occupying $0.5-0.7$ of head-width. Eye region gradually sloping; from in front, AME round set on common low tubercle;
ALE oval set high but not beside PME. Chelicerae partially porrect, parallel-sided, not apically coniform, with small low boss. Fangs without ridges or grooves, not elongate; tooth margins short, diagonal with 4 large and 1-2 smaller teeth on retromargin, $2-4$ on promargin. Maxillae long, ca. twice labium length; anterior ectal face truncate, outer edge broadly rounded; lunate depression on midectal face, basally truncate; serrula in long curving line. Labium clearly longer than wide, no strong grooves/ depressions, apically truncate, not convergent. Sternum shield-shaped, margins with deep scallops for anterior inner corner of coxa II, III; posteriorly blunt.
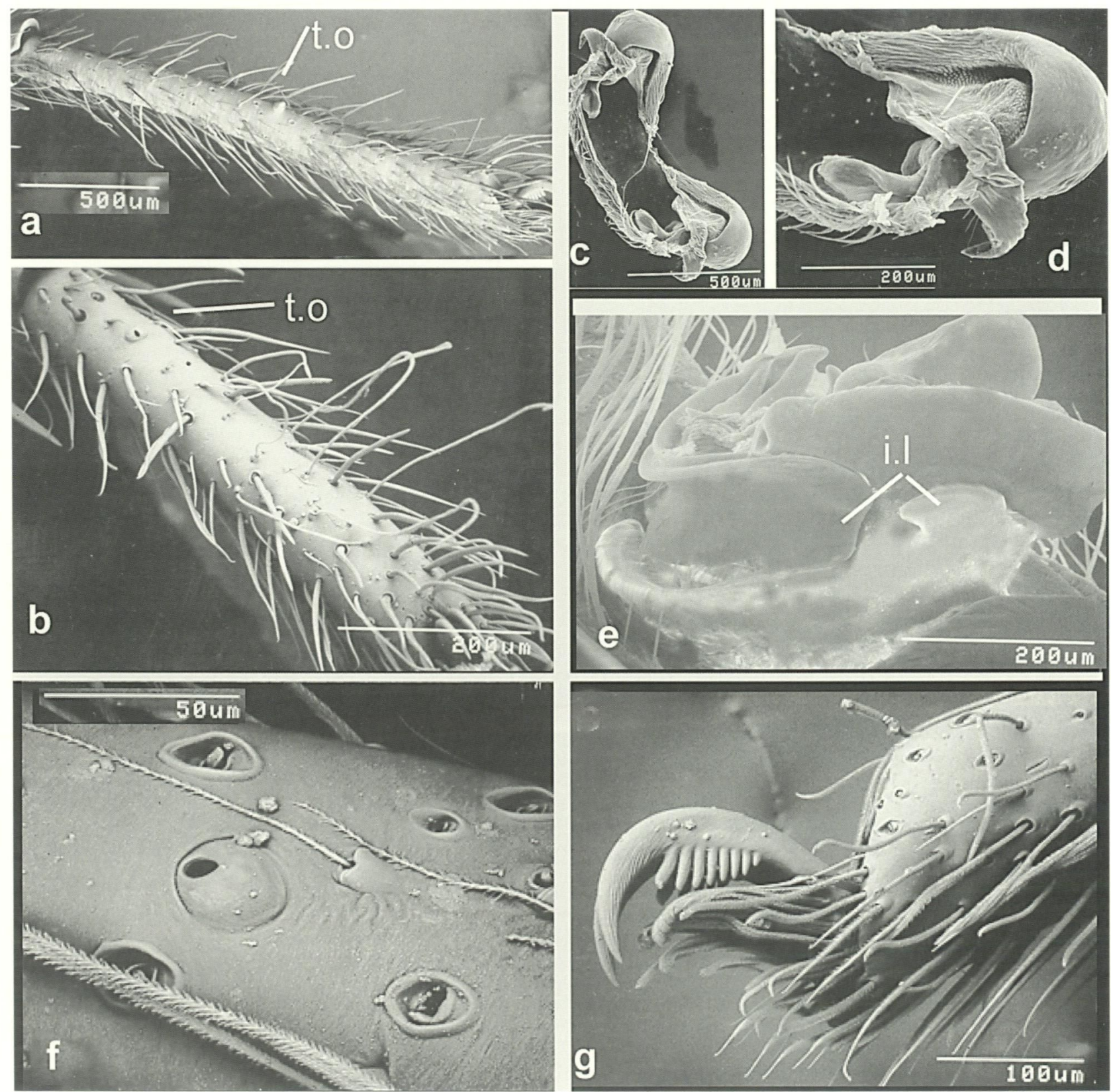

Figure 3 Amauropelma, females, scanning electron micrograph: a, A. anzses sp. nov., tarsal organ (t.o.) leg I; b, A. trueloves sp. nov., tarsi I showing position of tarsal organ (t.o) and enlarged dorsal setae; c, d, A. rifleck sp. nov., epigyne, dorsal face; e, A. mcilwraith, lateral palp and bulb showing interlocking lobes (i.1); f, g, A. hasenpuschi, female, leg I, showing tarsal organ and prostrate trichobothria (f), and claws and tufts (g). 
Legs. Formula 4123 (in all measured females save A. hasenpuschi $=4132$, most males) or 4132 (males of A. trueloves, A. bluewater, A. gayundah, A. rifleck, $A$. wallaman). Coxae all similar in shape, basally with small but distinct precoxal sclerites (inner triangular extensions), posterior more distinct than anterior; large distinct retrocoxal hymen basally on I, no opposed setae evident on prolateral II. All trochanters deeply notched. Hairs all simple. Scopula entirely absent except in $\$ A$. undara and $\delta$ $A$. anzses. Pilosity generally light. Spines: all strong; strong paired spines ventrally on tibia (4-5 pairs) and metatarsi ( 3 pairs) I, II, not or barely overlapping on tibiae; long, strong and overlapping on metatarsi; weakest on femora, absent on patellae I, II; proventral spine on femora absent or weak on femora I, III, and sometimes IV. Basal pair of spines on ventral tibiae I, II well-separated from base. Common spine pattern: I, II: pa, 0; ti v2.2.2.2.2; me v2.2.2. III, IV: ti v2.2.2. Preening combs absent. Claw tufts dense, narrow but set well below long claws. Two elongate claws with few small teeth basally; claws relatively longer on III, IV. Cuticle surface more or less smooth. Trichobothria in 2 rows on tarsi, irregular row on metatarsi, diffused band on tibiae. Tarsal trichobothria of two types: adpressed and erect (Figure 10b). Adpressed with more widely spaced microfilaments, base and aperture smaller than for erect trichobothria; base roughly triangular and aperture not on raised dome. Erect trichobothria larger; bases semicircular and aperture on raised dome often with distal transverse ridge; both types have 2-4 transverse ridges which may or may not be continuous across base. Trichobothria not in lengthening row on tarsi. Tarsal organ low, set in basal 1/6th of tarsi, ovate on III, IV, mid-distal on I, II, delimited by shallow encircling groove and distal acentric ovoid aperture; aperture very large in $A$. undarain; in $A$. anzses (Figure $10 \mathrm{c}$ ) and $A$. mossman, tarsal organ is distinct raised cup with large aperture dominating distal vertical face; in $A$. monteithi, tarsal organ an elongate inclined rod with lobular apex with large aperture (Figure 23b). Tarsi dorsally with two irregular lines of very large, thick setae with enlarged bases (Figure $3 b$ ).

Female palp: claw dentate, long, without tuft; pair of spines ventrally on tarsi predistally, also on cymbium (some species) and also probasally on tibiae.

Spinnerets very small, short; colulus absent; ALS about twice size of PLS; PMS (female) small, cylindrical, apical segments domed.

Male palp: patella with coniform process off each corner of patellar fold; tibia with retrodorsal conical mound bearing bifurcate apophysis. Cymbium boat or leaf-shaped with strong probasal lobe and retrobasal lobe or ridge, bent strongly at base of apical cone; weak scopula sometimes dorsally on cymbium; cymbium not deep, without retrobasal groove; apically conical, two spines ventro-apically. Subtegular-tegular locking lobes present; tegulum C-shaped; embolus a large curving plate, directed clockwise, with $2-4$ deep ventral ridges narrowing distally; sperm duct opens on back of plate (Figures 6c, 33d,e); median apophysis arising from large, central, pallid, unsclerotised region, ladle-like; conductor large, distal, membranous.

Epigyne a large trilobed plate with 2 horn-like processes (=teeth) below and behind lateral lobes; lateral and anterior plate margins rolled under (Figure 3c, d); anterior septum present only in $A$. claudie; simple spermathecae with anterior copulatory fossae behind plate; insemination duct follows curve of lateral lobe and enlarges distally to spermathecae; fertilisation duct posterior horn-like. In $A$. undara, an epigynal plug was present on one side of the epigyne under the anterior outer quadrant of the plate.

Tracheae (in $A$. rifleck) are two very short tubes limited to posterior $1 / 6$ of abdomen and arising from a common lumen. Tracheal spiracle short, barely evident.

\section{Remarks}

Amauropelma shares with Janusia the elongate form of the tarsal claws, the shape of the carapace, leg spination, spinneret configuration, and leg pilosity, and absence of leg scopula. Amauropelma differs from Janusia in that the tarsal organ or rod is basal rather than subdistal and the opening of the organ is circular not triangular. Also, in Amauropelma, the epigynal plate is wide with two 'wings' rather than long; in Amauropelma, the copulatory fossae are in front of the leading edge of the plate, whereas in Janusia they are lateral. In Janusia, the lateral teeth are processes extending from the lateral epigynal sides whereas in Amauropelma they begin internally just behind the copulatory fossae and extend back and out. Also, in Janusia, the maxillae are apically divergent (according to Gray, 1973, figure 2) whereas in Amauropelma they are parallel-sided.

Unnamed ctenids with eyes from caves in Western Australia strongly resemble Janusia. In these species, the lateral epigynal teeth are produced from the distal edge of the epigyne, the relative eye sizes are different $(\mathrm{ALE}=\mathrm{AME}<\mathrm{PME}$ $=$ PLE) and the ALE are closer to the PLE than PME but not on a common mound.

Amauropelma also shares similarities with the South African Phanotea Simon in the form of the male palp which also has a scooped median apophysis and the embolus apically divided into two divisions (see Griswold, 1994). Unlike Phanotea, Amauropelma has claw tufts and lacks both a third claw and the tibial crack in males; most species also lack leg scopula. Moreover, unlike Phanotea, 


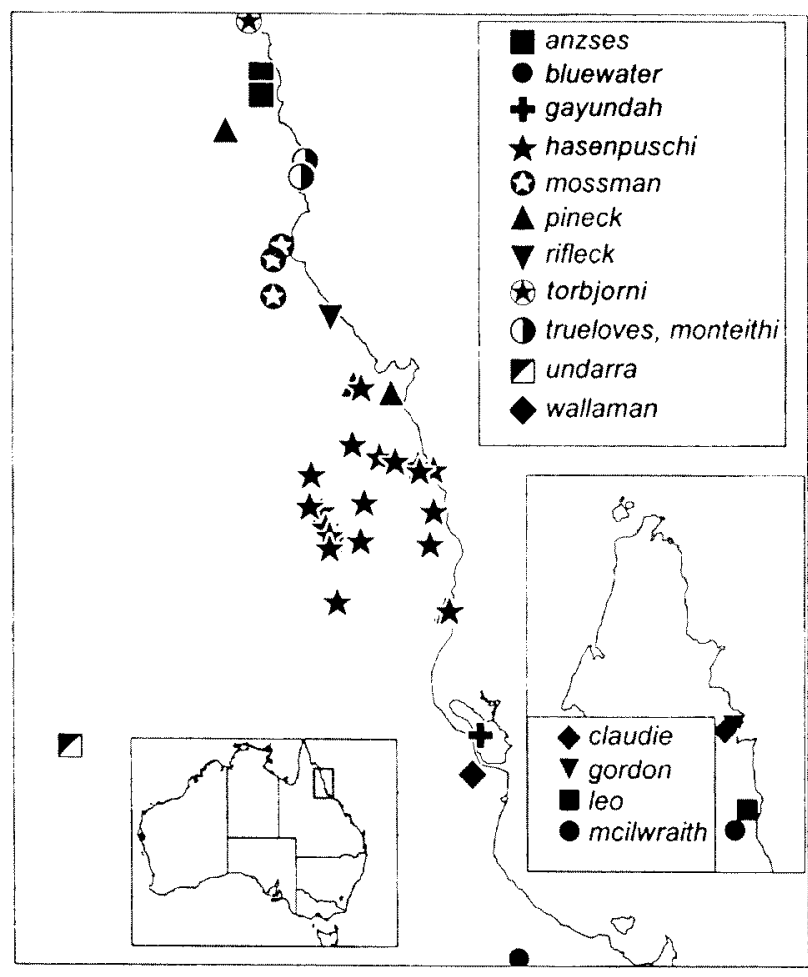

Figure 4 Amauropelma records, north-eastern Queensland: inset, Cape York.

Amauropelma has a patellar spur on the male palp, a pro- and retrobasal cymbial process, and a conically tapered cymbium.

\section{Distribution and Habitat}

Most species are found in lowland rainforests from Iron Range $(200 \mathrm{~km}$ south of the tip of Cape York) to just north of Townsville, north-eastern Queensland; one species lacking eyes has also been taken from caves west of Townsville.

\section{Phenology}

Males of Amauropelma have been taken throughout the year in $A$. trueloves, in which sampling has been year-long, five males were taken in winter (June). Hence, the generally accepted notion that araneomorphs are summer maturing must be tested with each genus.

\section{Relationships}

Relationships with taxa outside of Australia are complex principally because hypotheses about relationships among lycosoid genera are in a state of flux. On an ABRS funded visit to European and American Museums, RJR examined diverse genera while surveying the retrocoxal hymen. Some data presented here derive from that study.

Amauropelma was added to the data set of Griswold (1993) along with the retrocoxal hymen, the predistal tarsal fracture (see Raven, 1998) and the (dorsoventral) depth of the cymbium. Using the same character coding and Hennig86 commands as Griswold (1993), one tree was found. The tree placed Amauropelma in the ctenoid clade as the sister group of Ctenus and Phoneutria. This coincides with conclusions formed following discussions with Diana Silva: that Amauropelmalain is a ctenid. However, the plesiomorphic condition of the eyes in Amauropelma is a mildly recurved front row (with ALE beside AME, and not beside PME as in ctenids); hence, its inclusion in the Ctenidae completely compromises the traditional diagnostic character of the group.

Amauropelma seems most closely related to the African-Asian genus Thoriosa Simon but differs in the presence of a patellar spur on the male palp, the basal tarsal organ and in the similar sizes of the eyes; in Thoriosa, the AME are much smaller than the PME (Benoit, 1976). This relationship was suggested by Silva who will explore that question more deeply.

\section{Other similar taxa}

Three other genera show some indication of a relationship which Amauropelma. The New Zealand amaurobiid Maloides cavernicola (Forster and Wilton, 1973, holotype in New Zealand Arthropod Collection, examined) is known only from a single, three-clawed, female. However, in the eye group size (reduced), small eyes, two recurved (as figured, Figure 947, not procurved as described) eye rows, epigyne with lateral teeth and median plate, and simple spermathecae, the genus is similar to Amauropelma.

The Chilean 'amaurobiid' Emmenomma has 3 claws (no tufts), a scooped median apophysis and lateral teeth on the epigyne. It may also belong in this group. Third, Lehtinen (1967: 317) commented on presumed adaptations to cave living of some species of Phanotea - length of legs and trichobothria, reduced colour pattern, size of eyes and lengthening of the straight distal part of the paired tarsal claws - all found in Amauropelma.

\section{Cladistics of Species}

Within the genus, two characters show phylogenetically interpretable differences: the curvature of the front eye row and the development of the male patellar apophyses on the palp.

The front row eye curvature varies from almost straight from above to clearly curved to the extent that the ALE are decidedly higher than the AME and approach the PME (see A. claudie, Figure 14f). The outgroup condition is a more or less straight line and the ALE set beside the AME. Hence, clearly, the plesiomorphic condition of the front row curvature is more or less straight and the genus should not qualify on that character to be included in the Ctenidae. 
The palpal patella has a retrolateral invagination in many araneomorph genera. In Amauropelma males, the distal corners of the invagination form processes varying from small cones ( $A$. bluewater) to at least one large tube ( $A$. gordon). The outgroup condition (absence) suggests that the character is a synapomorphy of the genus.

Two species-groups are recognisable by a combination of characters: in the leo group, the eyes and overall eye group are small, the carapace lacks pattern and the spiders are larger; in the trueloves group, the eyes are larger and the group is wider relative to the head width, the spiders are smaller and the carapace has distinct lateral dark bands.

\section{Morphology}

Tarsal organ and rod. Typically, the tarsal organ is distal on the tarsi in spiders. When in the form of a rod in mygalomorphs (Ixamatus, Xamiatus; Raven, $1981)$ it is also distal. Araneomorph genera with a tarsal rod include the gradungulids Tarlina and the amaurobiids Otira and Storenosoma (e.g. Davies, 1986). Davies (1986: 240) noted that in Otira the rod is distal in New Zealand species and basal (ca. 0.25 length) in Australian species. The homolgy of the diverse rods is not presumed but simply the elevation of the receptive component off the tarsal surface. In $A$. monteithi, the rod is in the same location as the tarsal organ in other species and also there is no other tarsal organ; hence, in this case the rod and organ are presumed homologues. On all legs, the rod is at the basal 1/5.

The retrocoxal hymen (first reported by Raven, 1998 ) is readily observed by light microscopy on a medium-sized spider of the genus Cheiracanthium in which it is evident as a pallid unsclerotised circle on the ental half of the retrolateral face of coxa $I$. The predistal tarsal fracture (first reported by Raven, 1998) when present, occurs on all leg tarsi and is sometimes more easily seen in lateral view by light microscopy as a pallid diagonal zone predistally on the tarsus.

The embolus is unlike any thus far seen in Australian miturgids, zorids, clubionids, cycloctenids, pisaurids, or other ctenids. It consists of a broad flattened plate with several high curving ridges that distally converge and presumably 'lock' into the epigynal teeth or horns. The physical sizes are similar, at least for the distal portion of the embolus. The base of an epigynal horn is about 90 um wide and 200um long; the embolus tip is about $50 \mathrm{um}$ long and $20 \mathrm{um}$ wide. The opening of the sperm duct is discernible only under an SEM (Figures 6c, 33d, e) with the embolic plate so inclined as to allow examination of the dorsal apex of the plate. Prolaterally, the embolus is bounded by a curved ring, deeply concave externally, which probasally has an interlocking lobe with the tegulum (Figure $3 \mathrm{e}$ ).

\section{Species Included}

Amauropelma anzses sp. nov., A. bluewater sp. nov., A. claudie sp. nov., $A$. gayundah sp. nov., $A$. gordon sp. nov., $A$. hasenpuschi sp. nov., $A$. leo sp. nov., $A$. mcilwraith sp. nov., $A$. monteithi sp. nov., A. mossman sp. nov., $A$. pineck sp. nov., $A$. rifleck sp. nov., $A$. torbjorni sp. nov., A. trueloves sp. nov., A. undara sp. nov. and $A$. wallaman sp. nov.

\section{Etymology}

From the leg pattern which is reminiscent of the amaurobiid basic pattern; the gender is neuter.

\section{Key to species of Amauropelma}

Males (males of A. claudie unknown)

1. Eyes absent A. undara

Eyes present 2

2. Long tarsal rod present basally (Figure $23 b$ ) ..... A. monteithi

Tarsal rod absent or very low 3

3. Patellar apophysis long, vermiform (Figure 14c, 15b) A. gordon

Patellar apophysis short, coniform if present .. 4

4. Tarsal organ posteriorly raised with vertical distal face on mid-tarsi on I, basal 1.4 on II-IV (Figure 3a). A. anzses

Tarsal organ low, indistinct 5

5. Median apophysis subcircular with dominant cavity (Figure 12a). A. bluewater

Median apophysis clearly longer than wide.... 6

6. Patellar apophysis a prominent cone (Figure 31c)... A. torbjorni

Patellar apophysis a small sometimes bifid cone, if present. 7

7. Retrolateral paracymbial lobe distinctly produced (Figure 6a); cavity of median apophysis confined to apical one-third; large serrate keel on base of embolic plate (Figure 6d) ..................................................... A. trueloves

Retrolateral paracymbial lobe absent or thick but not well produced (Figures 18a, 24a) .....8

8. Tibial apophysis unequally bifid or single with small subdistal tooth (Figure $27 \mathrm{~b}$ )..... A. pineck

Tibial apophysis equally bifid (Figures 29b, d) .

\section{9}

9. Patellar apophysis is one fused bifid process (Figure 29c) ...................................... A. rifleck

Patellar apophysis is clearly two separate cones, if present (Figure 24c) ..................... 10

10. Median apophysis without lumen (Figures 13a, 27f) ............................................ A. gayundah 
Medan apophysis clearly with lumen (e.g. Figure 24a) 11

11. Embolic plate with thumb-like lobe below tip (Figure 20b) A. mcilwraith

Embolic plate without thumb-like lobe below tip.....

12. Tibial apophysis deeply divided for apical $2 / 3$ (Figure 34d) A. wallaman

Tibial apophysis shallowly divided in apical 1/3 (Figure 18b) ... 3

13. Median apophysis with dominant (prolateral) 'handle' (Figure 24a, b); embolic plate with low basal extra keel. A. mossman

Median apophysis with dominant (retrolateral) lumen; basal embolic plate without extra keel (Figure 18c) A. hasenpuschi

Females (females of $A$. gayundah unknown)

1. Eyes absent A. undara

Eyes present ... 2

2. Long tarsal rod present (Figure 23b)

A. monteithi

Tarsal rod absent or very low ... 3

3. Tarsal organ posteriorly raised with vertical distal face on mid-tarsi on I, basal 1.4 on II-IV (Figure 10c); scopula present on tarsi I, II .......

A. anzses

Tarsal organ low, indistinct; scopula absent on tarsi

4. Epigynal plate subquadrate (Figures 11c, 17f) ..

Epigynal plate clearly wider than long (Figure 5f) ... 6

5. From above, ALE about midway between PLE and AME (Figure 17d) A. hasenpuschi

From above, ALE clearly closer to PLE than ALE (Figure 11b) A. bluewater

6. Distal epigynal plate with concave cavity to lateral lobes (Figure 5f)

Distal epigynal plate with clearly convex lateral curve from distal edge to lateral wings (Figure 14d) A. gordon

7. Lateral horns set wide of and orthogonal to sagittal plane and extend well beyond lateral wings (Figure 14g) A. claudie

Lateral horns set close to lateral wings or hardly wider (Figure 5f)

8. Eye group small (Figure 19b) and AME clearly larger than PME. A. leo

Eye group large, normal (Figure 5a); $\mathrm{AME}$ and PME similar sizes (Figure $5 \mathrm{~d}$ )
9. From above, front edge of ALE in same line as those of PME or behind them; PME clearly larger than AME (Figure 25a).

From above, front edge of ALE clearly in front of those of PME; PME equal or subequal to AME (Figure 30a)

10. Tibiae I, II with 5 pairs of spines ventrally .... 11 Tibiae I, II with only 4 pairs of spines ventrally A. pineck

11. Overall epigyne only ca. 1.5-1.6 times wider than long (Figure 25c).... 12

Overall epigyne wider, ca. 1.9 times wider than long (Figure 5f); north of Daintree River and south of Bloomfield River A. trueloves

12. PME clearly bigger than ALE (Figure 25a); just south of Daintree River..... A. mossman

All eyes of similar size; just north-east of Townsville A. wallaman

13. Overall epigyne wide ca. 2.1 times wider than long with slender lateral horns (Figure 28b); Ellis Beach region A. rifleck

Overall epigyne longer $c a .1 .7$ times wider than long with fleshy lateral horns (Figure 30f); $\mathrm{Mt}$ Cook region A. torbjorni

\section{Amauropelma trueloves}

\section{Raven and Stumkat, sp. nov.}

Figures 1, 3b, 4-7, Table 1

\section{Material Examined}

Holotype

§., Pilgrim Sands, Cape Tribulation, Queensland, Australia, $16^{\circ} 00^{\prime} \mathrm{S}, 145^{\circ} 25^{\prime} \mathrm{E}, 24-29$ August 1988, R.J. Raven (QM S24194).

\section{Allotype}

Australia: Queensland: 1 \&, Cape Tribulation, 2 km WNW (Site 2), $16^{\circ} 04^{\prime} \mathrm{S}, 145^{\circ} 27^{\prime} \mathrm{E}$, pitfall, 23 September-7 October 1982, G. Monteith, D. Yeates, G. Thompson (QM S31355.

\section{Paratypes}

Australia: Queensland: 1 \% , 24-29 August 1988, R.J. Raven (QM S32917); 1 \&, 27 March-1 May 1996, P. Zborowski (QM S39107); 1 o, sieved litter, 13 October 1980, G. Monteith (QM S32888); 1 o, sieved litter, 14 October 1980, G. Monteith (QM S32885); 1 Q, sieved litter, 23 September 1982, G. Monteith, D. Yeates, G. Thompson (QM S32886); 1 \&, sieved litter, 2 October 1982, G. Monteith, D. Yeates, G. Thompson (QM S32887); 3 \%, pitfall, 31 May-28 June 1996, P. Zborowski (QM S41784); 1 q, 5 December 1995-4 January 1996, L. Umback (QM S39113); 1 d, pitfall, 31 May-28 June 1996, P. Zborowski (QM S39112); 2 o, pitfall, 31 May-28 


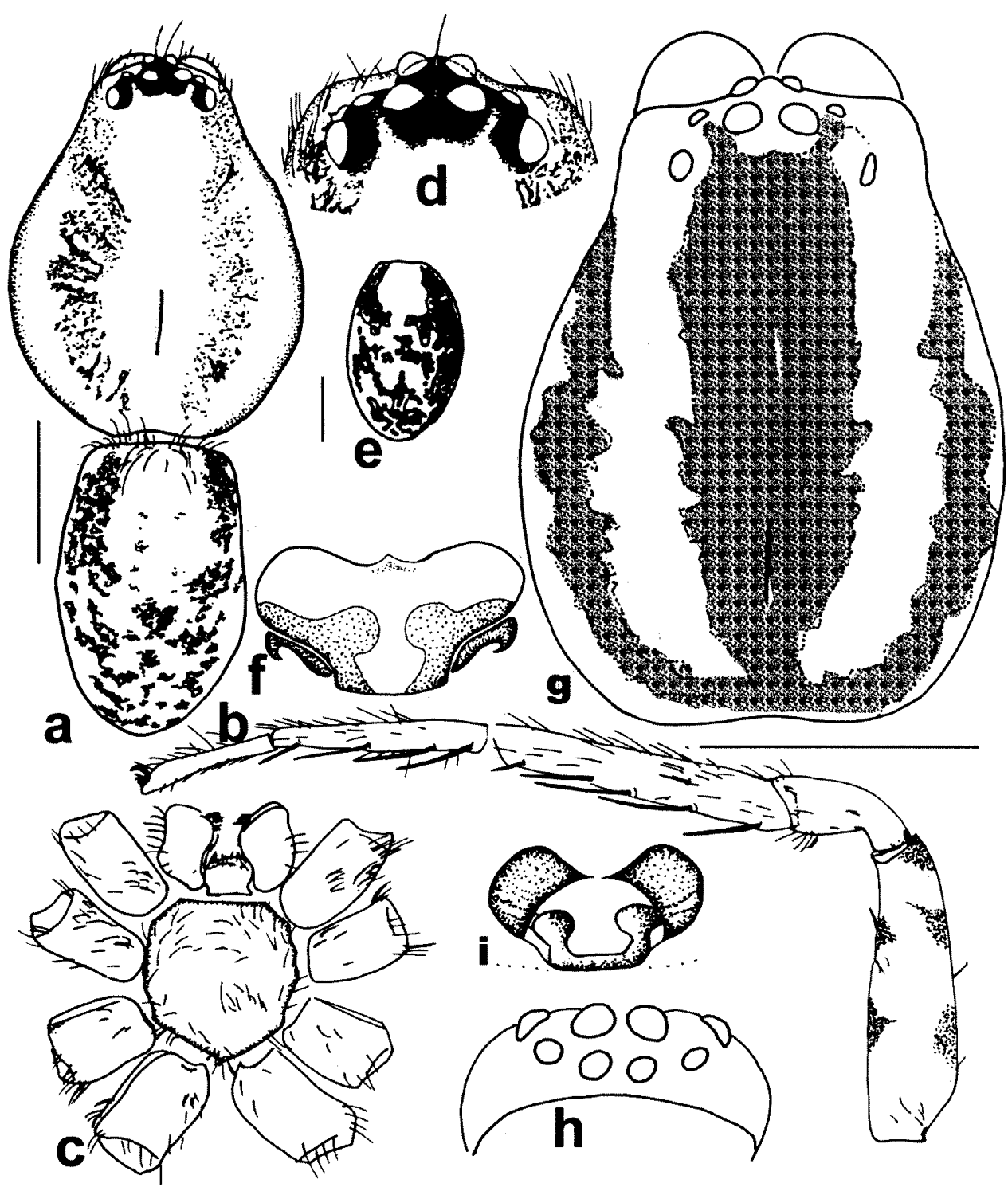

Figure 5 Amauropelma trueloves sp. nov.: holotype male, a-d: a, carapace and abdomen, dorsal view; b, leg I, prolateral view; $c$, sternum, maxillae, labium and coxae; d, eyes, dorsal view. e-i, female: e-h, allotype; e, abdomen, dorsal view; $f$, epigyne, external, ventral view; $g$, carapace and chelicerae; $h$, eyes, frontal view; $i$, epigyne (QMS41785, dorsal view. Scale lines $=1 \mathrm{~mm}$, except $f, i, 0.5 \mathrm{~mm}$.

June 1996, P. Zborowski (QM S39109); 1 \&, pitfall, 1-28 February 1996, L. Umback (QM S39116); 1 \%, sieved litter, January 1983, G. Monteith (QM S26902); 1 o, flight intercept trap, 1-28 February 1996, L. Umback (QM S39114); 1 o, flight intercept trap, 31 May-28 June 1996, P. Zborowski (QM S41782); 1 \&, pitfall, 31 May-28 July 1996, P. Zborowski (QM S39105); 1 , pitfall, 31 May-28 July 1996, P. Zborowski (QM S39106); 1 \&, pitfall, 31 May-28 June 1996, P. Zborowski (QM S39110); 1 q. pitfall, 5 December 1995-4 January 1996, L. Umback (QM S39118); 3 \& , pitfall, 27 March 1996-1 May 1996, P. Zborowski (QM S41785); 1 ¿, pitfall, 31 May-28 June 1996, P. Zborowski (QM S39111)); 1 \&, pitfall, 1-28 February 1996, L. Umback (QM S39117); 1 o, 1 \&, pitfall, 1-28 February 1996, L.
Umback (QM S41783); 2 ơ, sieved litter, 23 September-7 October 1982, G. Monteith, D. Yeates, G. Thompson (QM S32884); 1 \&, Noah Head, S. Cape Tribulation, $16^{\circ} 07^{\prime} \mathrm{S}, 145^{\circ} 27^{\prime} \mathrm{E}$, sieved litter, 16 October 1980, G. Monteith (QM S26904); 2 o, pitfall, 29 November 1992-17 April 1993, R., J. and S. Raven, P. and E. Lawless (QM S19769); 2 \&, pitfall, 21 July-29 November 1992, R. Raven, P. Lawless, E. Lawless, M. Shaw (QM S24038); 13 万, 3 \&, pitfall, 5 November 1991-20 July 1992, R. Raven, P. Lawless, M. Shaw (QM S24863, WAM T42600, AMNH, ANIC). All from rainforest at Cape Tribulation.

\section{Diagnosis}

Differs from A. monteithi in lacking a tarsal rod, having larger raised eyes and more pattern and 

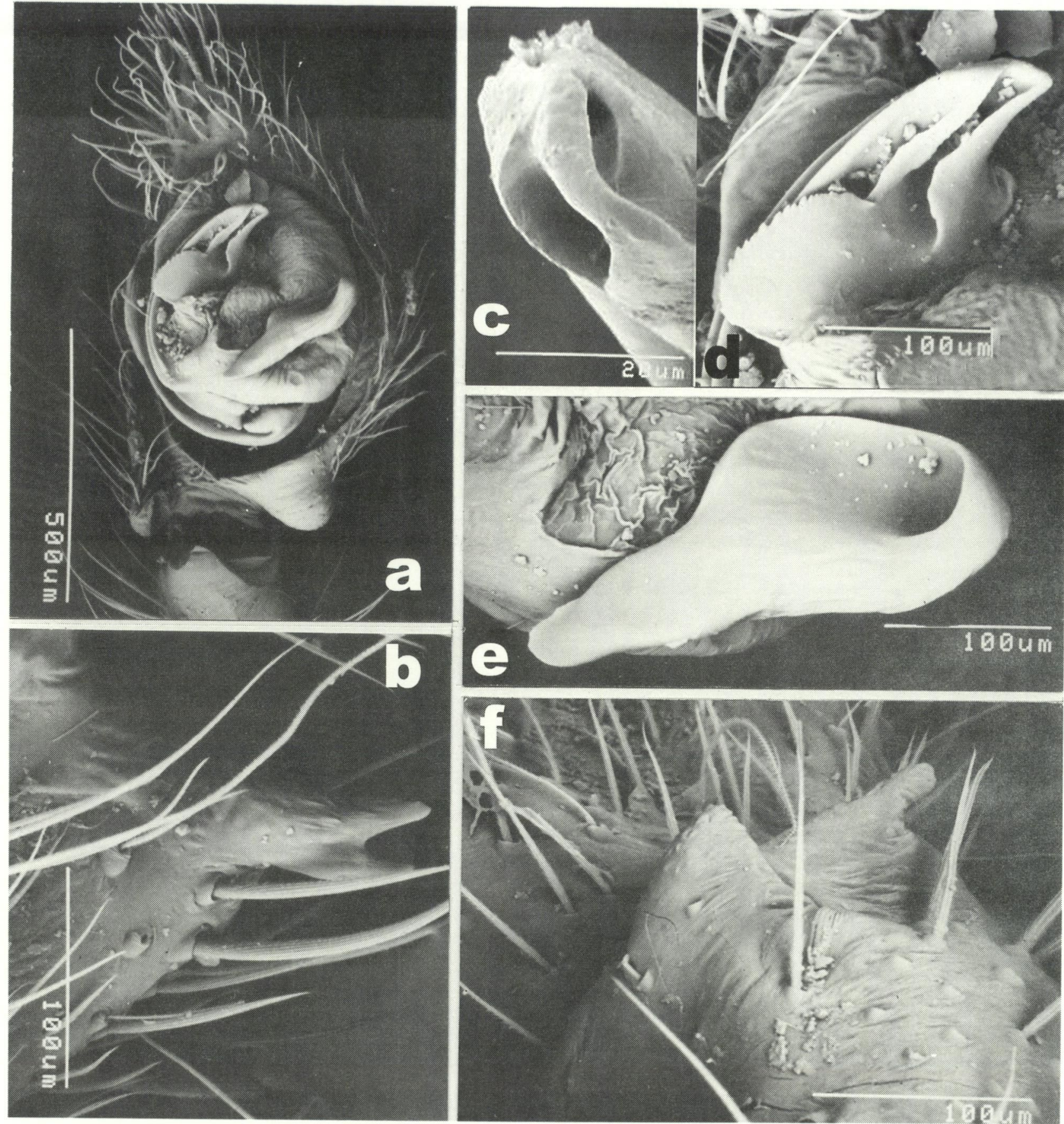

Figure 6 Amauropelma trueloves sp. nov.: male palp, ventral right, scanning electron micrographs: a, cymbium, bulb and distal tibia; b, tibial apophysis, ventral view; c, embolus tip showing spermatic outlet, axial view; $d$, embolic plate and conductor, ventral view; d, median apophysis, ventral view; f, patellar apophyses, retrolateral view.

from $A$. pineck in have 5 pairs of spines ventrally on tibiae I, II.

\section{Description}

\section{Holotype male (QM S24194)}

Carapace 2.46 long, 2.00 wide. Abdomen 2.07, 1.38 wide. Colour. Carapace and chelicerae orange with shadows along edge of carapace, beside fovea, and as slightly dark rings on legs as in 9 . Abdomen darker with pallid medial region anteriorly, broken open brown areas posteriorly; ventrally almost entirely pallid with darker spots laterally. Eyes all surrounded by black pigment. Carapace. Fovea begins at widest point. Eyes. AME:ALE:PME:PLE, 7:7:9:8. Spines. As for genus except as noted. I: fe pv1p1d3r1; ti p1. II: fe pv1p1d3r1; pa 0; ti p1; me p1. III: fe pv1p2d3r3; pa r1; ti p2d3r2; me p4r4v2.2.4 short pair medially. IV: fe pv1p2d3r2; pa r1; ti p2d3r2; me p5r5v2.2.2 short pair medially. Palp: fe p1d3, pa p1w, ti p2w, cymbium 2 distoventral. Legs. Scopula absent. Palp. Palpal patella with two clearly 
Table 1 Leg measurements of Amauropelma trueloves.

Holotype male (QMS24194).

\begin{tabular}{llllll}
\hline & Leg 1 & Leg 2 & Leg 3 & Leg 4 & Palp \\
\hline Femur & 2.00 & 1.69 & 1.69 & 2.23 & 0.92 \\
Patella & 0.92 & 0.77 & 0.85 & 1.00 & 0.31 \\
Tibia & 1.92 & 1.46 & 1.23 & 2.00 & 0.38 \\
Metatarsus & 1.46 & 1.31 & 1.38 & 1.92 & \\
Tarsus & 0.92 & 0.85 & 1.00 & 1.61 & 1.00 \\
\hline Total & 7.22 & 6.08 & 6.15 & 8.76 & 2.61 \\
\hline
\end{tabular}

Allotype female (QMS31355).

\begin{tabular}{llllll}
\hline & Leg 1 & Leg 2 & Leg 3 & Leg 4 & Palp \\
\hline Femur & 1.92 & 1.73 & 1.61 & 2.23 & 0.92 \\
Patella & 0.85 & 1.08 & 0.85 & 0.85 & 0.38 \\
Tibia & 1.69 & 1.38 & 1.15 & 1.85 & 0.46 \\
Metatarsus & 1.23 & 1.23 & 1.31 & 2.15 & \\
Tarsus & 0.65 & 0.73 & 0.92 & 1.15 & 0.69 \\
\hline Total & 6.34 & 6.15 & 5.84 & 8.23 & 2.45 \\
\hline
\end{tabular}
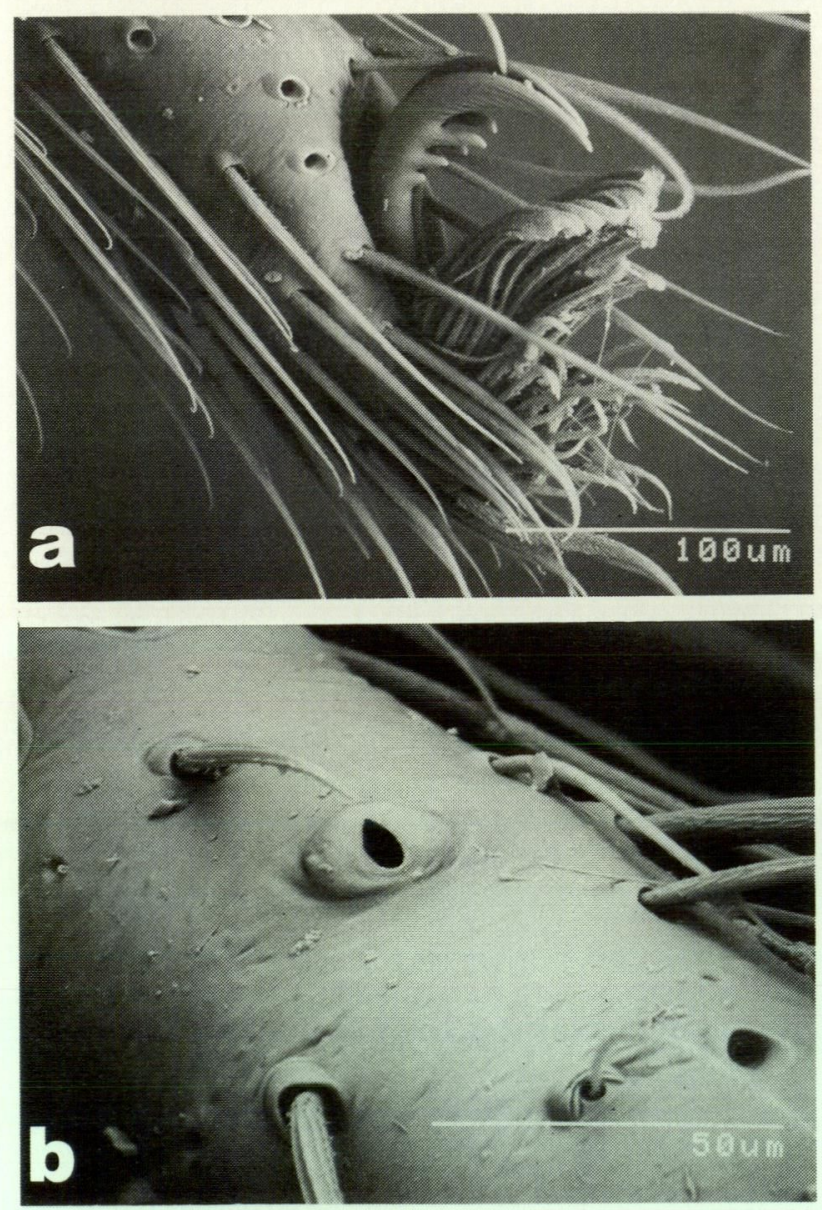

Figure 7 Amauropelma trueloves sp. nov. female, scanning electron micrographs: a, distal tarsus, paired claws and claw tufts; $b$, tarsal organ and trichobothium. equally sized divided cones on retrodistal corner; retrobasal cymbial lobe rounded, blunt bur distinctly produced; embolic plate short, beak-like. Median apophysis long, ladle-shaped with small lumen and long broad handle. Spinnerets. All very short or small, atrophied; PMS $=$ PLS $<0.5 x$ ALS; all with domed tips. Slight but evident space between ALS.

\section{Allotype female (QM S31355)}

Carapace 2.54 long, 2.08 wide. Abdomen 3.30, 1.92 wide.

Colour. More boldly marked than A. monteithi. Carapace yellow orange with narrow marginal bands on each side stopping before anterior constriction, wider dark band begins at AME across to anterior carapace edge and back beside fovea. A wide dark band medially down chelicerae; abdomen dark grey with large pallid medial area anterior and 3 paired patches behind and large patches laterally. Legs strongly banded; 3 bands on femora only distal unbroken dorsally by spines. One band on patella and two on each of tibia and metatarsus. Ventral abdomen pallid with roughly large paired areas of dark. Sternum, maxillae and labium yellow orange, chelicerae darker; irregular shadows medially on sternum. Eyes with dark bases. Carapace. Almost glabrous save for fine black hairs (and sheen). Paired setae along clypeal margin, single bristle between AME. Eyes. AME:ALE:PME:PLE, 7:8:9:9. Eyes seem relatively larger cf male. Chelicerae, maxillae and labium similiar to male. Spines. As for genus except as noted. I: fe pv1p1d3. II: fe p1d3. III: fe pv1p2d3r2; pa r1; ti p2d3r2; me p4r2v2.2.2. IV: fe pv1p2d3r1; pa r1; ti p2d2r2; me p4r4v2.2.2. Palp: fe p1d3; pa p1; ti p2; ta p3. Legs. Scopula absent. Trochanters deeply notched (notch as long as wide on IV less so on I but still deep). Sheen also present. Claws. 2, noticeably long and thin, 3-4 short teeth medially. Tufts small, below claw distinct. Epigyne. Externally a broad plate with two deeply encased broad lateral lobes and long curved horns. Copulatory ducts in horns.

\section{Variation}

Intensity of pattern on carapace, legs and abdomen of males varies from pallid to dark.

\section{Distribution and Habitat}

Known only from lowland rainforest near Cape Tribulation, north of Cairns, north-eastern Queensland.

\section{Etymology}

A noun in apposition from a local name for the area. 
Amauropelma anzses Raven and Stumkat, sp. nov. Figures $3 a, 4,8-10$, Table 2

\section{Material Examined}

\section{Holotype}

§. Mt Finnigan, site 1, Queensland, Australia, $15^{\circ} 47^{\prime} \mathrm{S}, 145^{\circ} 16^{\prime} \mathrm{E}$, pitfall, 4 December $1990-17$ January 1991, QLD Museum and ANZSES (QM S31360).

\section{Paratypes}

Australia: Queensland: $2 \hat{\delta}$, same data as holotype (QM S31359); 2 \%, same data as holotype except site 3 (QM S32918); 1 \%, same data as holotype except site 4 (QM S32919); 1 ठ , Big Tableland, $15^{\circ} 43^{\prime} \mathrm{S}, 145^{\circ} 16^{\prime} \mathrm{E}$, flight intercept trap, 20 December 1990-8 January 1991, ANZSES expedition (QM S32914).

\section{Diagnosis}

Differs from all other species in having leg scopula in males and in having a distinctly raised (but not a rod) tarsal organ.

\section{Description}

Holotype male (QM S31360)

Carapace 4.54 long, 3.77 wide. Abdomen 4.07, 2.69 wide.

Colour. Entirely yellowish, dark fine lines along strial margins on carapace. Eyes. AME:ALE: PME:PLE, 10:8:6:6. AME clearly the largest. Chelicerae. Promargin with 2 teeth; retromargin with

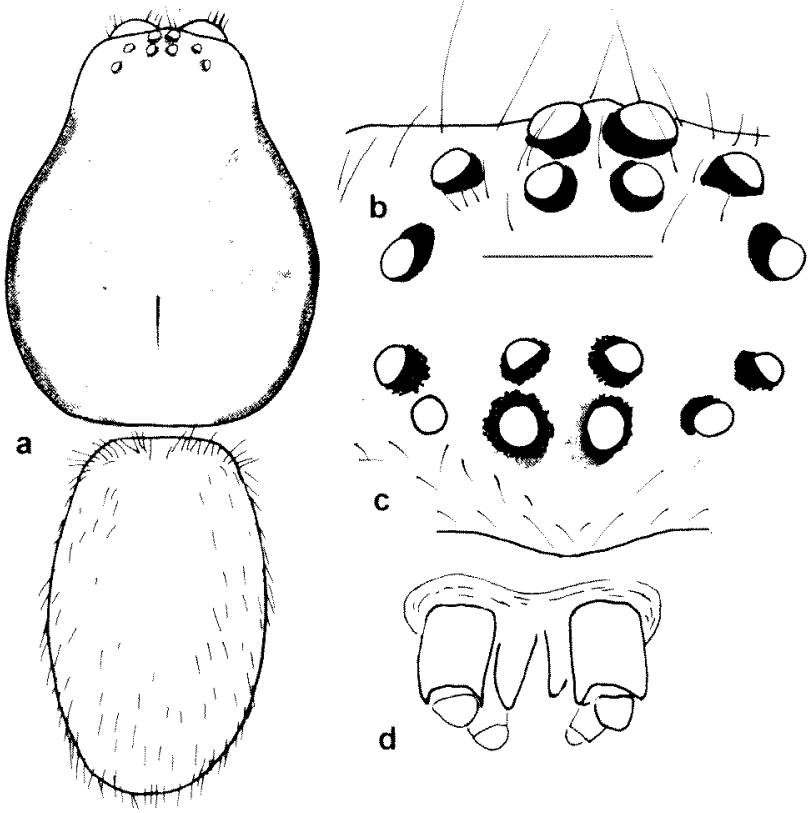

Figure 8 Amatropelma anzses sp. nov.: holotype male: $a$, carapace and abdomen, dorsal view; $b$, eyes, dorsal view; c, eyes, front view; d, spinnerets, ventral view. Scale line $=2 \mathrm{~mm}$ for a, $1 \mathrm{~mm}$ for $\mathrm{b}-\mathrm{d}$.
Table 2 Leg measurements of Amauropelma anzses.

Holotype male (QMS31360).

\begin{tabular}{llllll}
\hline & Leg 1 & Leg 2 & Leg 3 & Leg 4 & Palp \\
\hline Femur & 4.08 & 3.69 & 3.31 & 4.31 & 1.77 \\
Patella & 1.92 & 1.92 & 1.46 & 1.69 & 0.61 \\
Tibia & 4.08 & 3.31 & 2.92 & 4.08 & 0.77 \\
Metatarsus & 3.69 & 2.92 & 3.15 & 4.61 & - \\
Tarsus & 2.07 & 1.46 & 1.38 & 2.38 & 1.46 \\
\hline Total & 15.84 & 13.30 & 12.22 & 17.07 & 4.61 \\
\hline
\end{tabular}

4. Sternum. Posterior corners almost flat, others distinct but broad and mid-distal. Spines. As for genus except as noted. I: fe pv1 strong p2d3r2; ti p1r1; me p2r1. II: fe pv1p2d3r2; ti p1r1; me p3r2. III: fe pv1p2d3r4; pa r1; ti p2d3r2; me p2+2l r3+2l v2.2.2. IV: fe p4d3 r2; pa r1; ti p2d2r2; me p2+21 r3+2p v5.2. Palp: fe p1d1.2; pa p1w; ti p2r1. Cymbium, 2 small subapical. Legs. Distinct raised lobe on tarsus almost medial on I, at basal $1 / 4$ on II-IV. Trochanteral notches deep. Scopula: tarsi I-II, entire; IV with line of emergent setae; metatarsi I-III entire; IV tripartite. Claws. 3-4 short teeth on I, 1 small on IV, tufts narrow distinct. Trichobothria. 2 rows on tarsi, irregular row on metatarsi, diffused band on tibiae. Palp. Patella with two separate retrodistal cones of dissimilar height, upper cone larger. Tibia incrassate, distodorsally with dissimilarly bifurcate apex (ventrally retro-laterally), two distal extensions on tibia prolaterally interact with broadly rounded probasal cymbial lobe; retrobasal cymbial lobe a distinct process from basal cymbial flange. Spinnerets. Wide apart; ALS about 1.2 diameter apart, PMS long.

Female

Unknown.

\section{Distribution and Habitat}

Known only from rainforest at Mt Finnigan and Big Tableland, north-eastern Queensland.

\section{Etymology}

An acronym of Australian New Zealand Scientific Exploration Society (ANZSES) for a group that included some of the collectors.

\section{Amauropelma bluewater}

Raven and Stumkat, sp. nov. Figures 4, 11, 12, Table 3

\section{Material Examined}

Holotype

$\delta$, Bluewater Range, Queensland, Australia, 

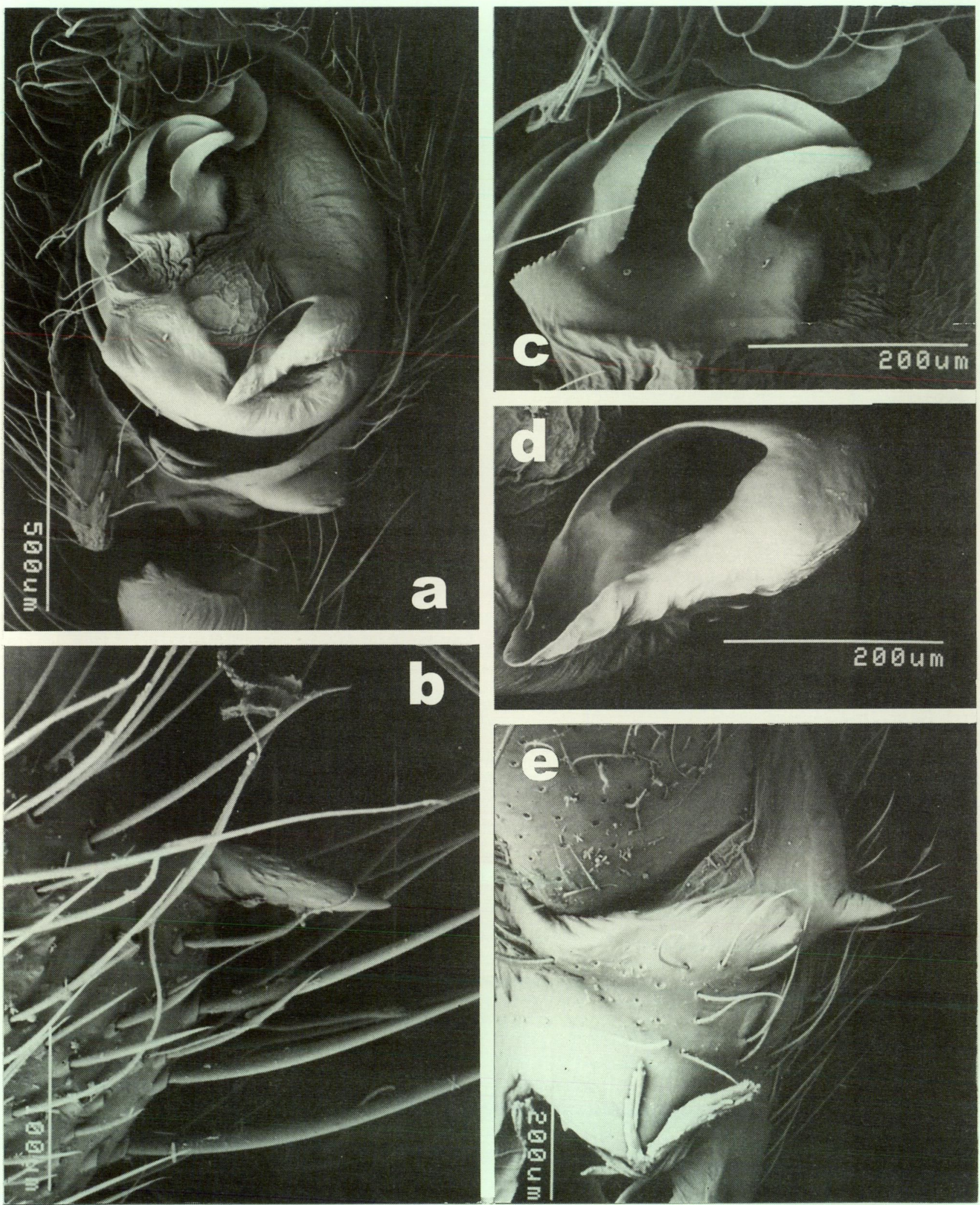

Figure 9 Amauropelma anzses sp. nov.: male palp, ventral right, scanning electron micrographs: a, cymbium, bulb and distal tibia, ventral view; b, tibial apophysis, ventral view; c, embolic plate and conductor, ventral view; d, median apophysis, ventral view; e, patellar apophyses, disto-retrolateral view. 

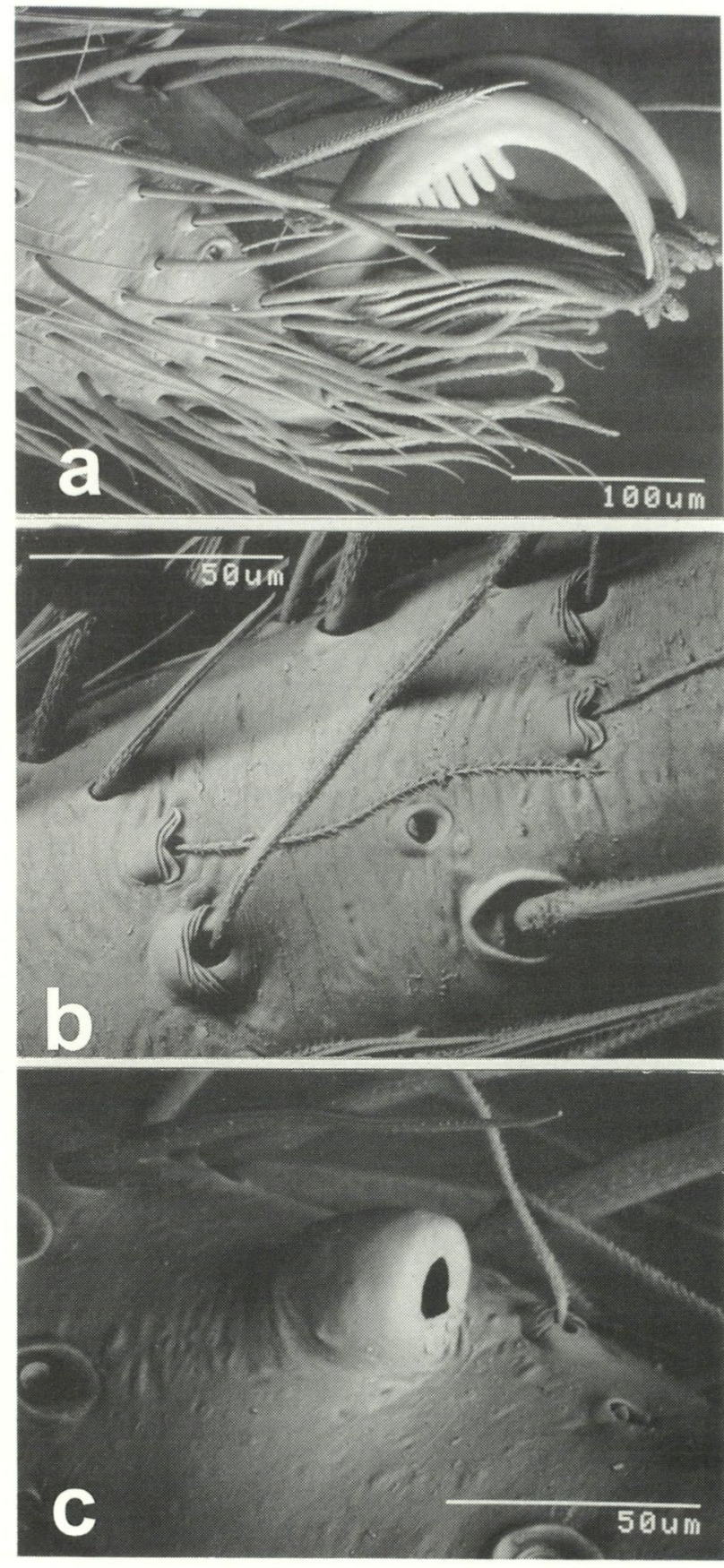

Figure 10 Amauropelma anzses sp. nov., male, scanning electron micrographs: a, distal tarsus, paired claws and claw tufts; $b$, adpressed and erect trichobothria; $c$, raised tarsal organ with distal aperture.

$19^{\circ} 10^{\prime} \mathrm{S}, 146^{\circ} 22^{\prime} \mathrm{E}$, rainforest, sieved litter, 7 December 1986, G. Monteith, G. Thompson (QM S31366).

\section{Allotype}

Australia: Queensland: $q$, same data as holotype (QM S31367).

\section{Paratype}

Australia: Queensland: $1 \delta$, Mt Halifax, SE ridge, $19^{\circ} 17^{\prime S}$, $146^{\circ} 23^{\prime} \mathrm{E}, 21$ March-10 May 1991, D. Cook (QM).

\section{Diagnosis}

Males differ from those of all other species in the small size of the patellar process on the palp and the rounded median apophysis and females from $A$. hasenpuschi in having only 4 pairs of spines ventrally on tibiae I, II.

\section{Description}

\section{Holotype male (QM S31366)}

Carapace 2.76 long, 2.07 wide. Abdomen 4.15, 2.77 wide.

Colour. Orange brown with fine dark lines along striae, legs without bands. Abdomen dorsally dark, laterally with 5 irregular ovoid areas, centrally with dark intervening triangles, laterally pallid with dark mottling; ventrally entirely unmarked. Dark shadows around eyes. Eyes. AME:ALE:PME:PLE, 7:6:6:6. ALE lighter colour. All of similar size. Anterior edge of ALE almost in line with front edge of PLE. Spines. As for genus except as noted. I: fe pv1p1d3r1; ti r1. II: fe pv1p1d3r2; ti p1r1; me p1r1. III: fe pv1p2d3r3; pa r1; ti p2d3r2; me p2+21 v2.2.2.(2.1) close. IV: fe pv1p2d3r1rv1; pa r1; ti p2d3r2; me p2+2l d1r2+2l v1.2.2.2.2. close. Palp: fe p1d1.2; ti p1.1; cymbium subapical 2. Legs. Tarsal organ at basal $1 / 6$; tarsal trichobothria c.4-6 dorsally in basal $1 / 2$. Spinnerets. Set close together. Claws. Tufts distinct small. STC IV and teeth medial, ca. 6 on I. Palp (Figure 12). Patella with two low distal processes retrolaterally; tibia with single retrodorsal apophysis deeply divided into two

Table 3 Leg measurements of Amauropelma bluewater.

\begin{tabular}{lccccc}
\hline \multicolumn{7}{l}{ Holotype male (QM S31366). } \\
\hline & Leg 1 & Leg 2 & Leg 3 & Leg 4 & Palp \\
\hline Femur & 2.08 & 1.92 & 1.76 & 2.24 & 0.80 \\
Patella & 1.04 & 0.92 & 0.80 & 1.00 & 0.48 \\
Tibia & 1.88 & 1.48 & 1.28 & 2.00 & 0.40 \\
Metatarsus & 0.92 & 0.92 & 1.52 & 2.24 & - \\
Tarsus & 0.84 & 0.80 & 1.32 & 1.32 & 0.88 \\
\hline Total & 6.76 & 6.04 & 6.24 & 8.80 & 2.56 \\
\hline
\end{tabular}

Allotype female (QMS31367).

\begin{tabular}{llllll}
\hline & Leg 1 & Leg 2 & Leg 3 & Leg 4 & Palp \\
\hline Femur & 1.76 & 1.76 & 1.60 & 2.20 & 1.12 \\
Patella & 0.96 & 0.80 & 0.80 & 0.96 & 0.48 \\
Tibia & 1.60 & 1.40 & 1.20 & 1.92 & 0.40 \\
Metatarsus & 1.24 & 1.20 & 1.20 & 2.00 & \\
Tarsus & 0.60 & 0.56 & 1.08 & 1.08 & 0.68 \\
\hline Total & 6.16 & 5.72 & 5.56 & 8.16 & 2.68 \\
\hline
\end{tabular}




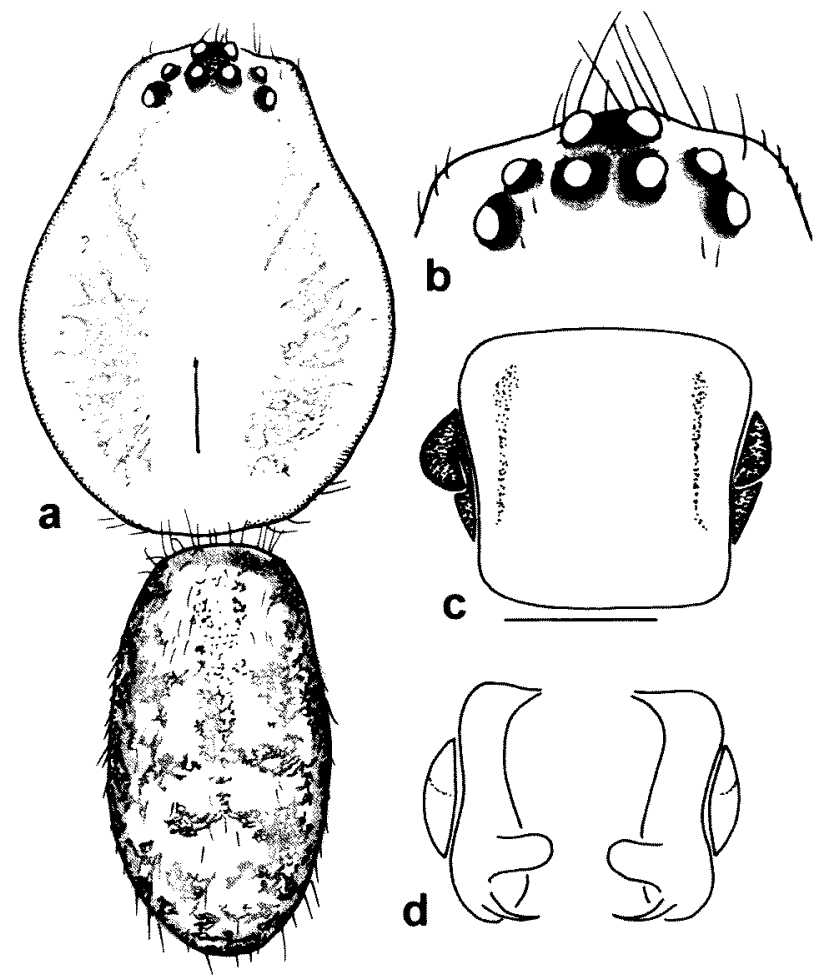

Figure 11 Amauropelma bluewater sp. nov.: holotype male: a, carapace and abdomen, dorsal view; b, eyes, dorsal view. c, d, allotype female: $c$, epigyne, ventral view; $d$, epigyne, dorsal view. Scale line $=2 \mathrm{~mm}$ for $a, 1 \mathrm{~mm}$ for $b$, $0.21 \mathrm{~mm}$ for $\mathrm{c}, \mathrm{d}$.

strong prongs. Cymbium with large rounded retrobasal lobe. Median apophysis almost circular with large central lumen.

\section{Allotype female (QM S31367)}

Carapace 2.76 long, 2.07 wide. Abdomen 3.53, 2.39 wide.

Colour. Carapace and legs yellow brown; abdomen pattern faded. Eyes. AME:ALE:PME:PLE, 6:5:5:6. ALE slightly closer to PLE than AME; from above, front edge of ALE just in front of that of PME; from in front, ALE set almost above top line of AME. Back row just form 2 lines. Chelicerae. Promargin with 3 teeth; retromargin with 1 small basal and 4 large distal teeth. Legs. Tarsal organ from distal $1 / 3$ (I) to basal 1/8-1/6(II-IV). No scopula. Claws. With 3 (III, IV) or 5-6 (I, II) teeth. Spines. I: fe pv1p2d3; ti v2.2.2.2. II: fe p1d3; pa 0 ; ti v2.2.2.2. III: fe pv1p2d3r2; pa r1; ti p2d3r2v2.2.2; me p1.1.2r1.1.2.v2.2.2. IV: fe p2d3r1; pa r1; ti p2d2r2v2.2.2; me p3r3v2.2.2. Palp: fe p1d1.2; pa p0; ti p2d1; ta p2r1. Epigyne. A broadly subquadrate plate with broad fleshy lateral horns closely adpressed to lateral plate.

\section{Distribution and Habitat}

Known only from rainforest at Bluewater Range, and Mt Halifax, just north of Townsville, northeastern Queensland.

\section{Etymology}

A noun in apposition taken from the name of the type locality.

Amauropelma claudie Raven and Stumkat, sp. nov. Figure 4, 14g, Table 4

\section{Material Examined}

\section{Holotype}

․ West Claudie River, Iron Ranage, Queensland, Australia, $12^{\circ} 44^{\prime} \mathrm{S}, 143^{\circ} 13^{\prime} \mathrm{E}$, rainforest, sieved litter, 6 December 1985, G. Monteith (QM S32901).

\section{Diagnosis}

Females differ from those of all other species in the lateral horns of the epigyne extending wide of the lateral wings and being distal.

\section{Description}

\section{Holotype female (QM S32901)}

Carapace 2.28 long, 1.88 wide. Abdomen 2.40 long, 1.72 wide. Total length 5 .

Colour. Carapace yellow brown with dark marginal and central broad shadow; chelicerae and legs yellow brown with bands or pattern. Abdomen with faded pattern but 3 large paired ovoid areas anteriorly and 3 transverse pallid bands posteriorly. Eyes. 2.4.2. PME $>A L E=P L E>P M E ; A M E$ set forward on common tubercle; all but ALE fully encircled by black ring, ALE with dark crest dorsally. From above, front of ALE in line with that of PME but ALE roughly equidistant from ALE and PLE; from front, front of ALE in line with top of AME. Eye group $=0.62$ of headwith; PME $=8 \%$ of headwidth. Chelicerae. Retromargin with 4 large and 1 small basal tooth; promargin with 3 teeth, middle largest. Legs. Tarsal organ at 2/5 from distal on I, II, at basal $1 / 6$ on III, IV. Scopula absent. Spines. As for genus except as noted. I: fe pv1p1d2; pa v1.

Table 4 Leg measurements of Amauropelma claudie.

Holotype female (QM S32901).

\begin{tabular}{lccccc}
\hline & Leg 1 & Leg 2 & Leg 3 & Leg 4 & Palp \\
\hline Femur & 1.60 & 1.60 & 1.44 & 2.00 & 0.80 \\
Patella & 0.88 & 0.80 & 0.60 & 1.76 & 0.32 \\
Tibia & 1.40 & 1.20 & 1.00 & 1.60 & 0.48 \\
Metatarsus & 1.12 & 1.00 & 1.20 & 1.92 & \\
Tarsus & 0.60 & 0.52 & 1.04 & 1.04 & 0.64 \\
\hline Total & 5.60 & 5.12 & 5.00 & 8.32 & 2.24 \\
\hline
\end{tabular}



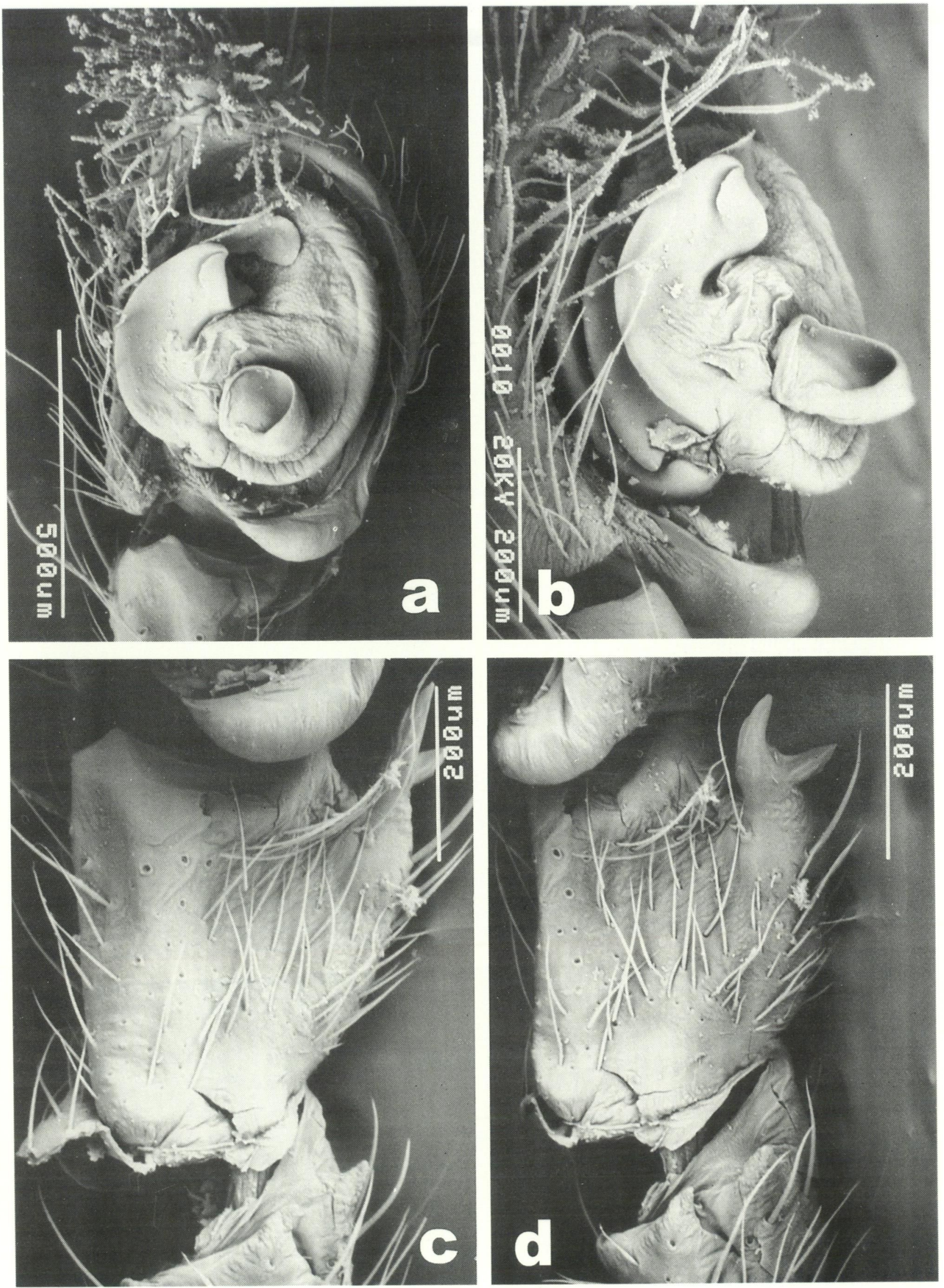

Figure 12 Amauropelma bluewater sp. nov.: male palp, ventral right, scanning electron micrographs: a, b, cymbium, bulb and distal tibia, ventral view (a), and prolateral view (b) showing extended median apophysis; c, d, patella and tibia, ventral view (c), retroventral view (d). 
Table 5 Leg measurements of Amauropelma gayundah.

Holotype male (QMS32990).

\begin{tabular}{llllll}
\hline & Leg 1 & Leg 2 & Leg 3 & Leg 4 & Palp \\
\hline Femur & 1.72 & 1.60 & 1.40 & 1.88 & 0.64 \\
Patella & 0.80 & 0.76 & 0.64 & 0.76 & 0.32 \\
Tibia & 1.44 & 0.84 & 1.80 & 1.60 & 0.36 \\
Metatarsus & 1.20 & 1.08 & 1.12 & 1.80 & \\
Tarsus & 0.56 & 0.60 & 0.96 & 0.96 & 0.84 \\
\hline Total & 5.72 & 4.88 & 5.64 & 7.00 & 2.16 \\
\hline
\end{tabular}

II: fe p1d3; me v2.2.2. III: fe p2d2r1; pa 0 ; ti p2d3r2; me p3r3v1.2.2. IV: fe p2d3r1; pa r1; ti p2d3r2; m3 p3r3v1.1.1.1.2. Palp: fe p1d1.2; pa p1; ti p1d1; ta p2.1 Epigyne. With wide plate, anteriorly indented and large lateral horns extending widely distally on plate.

\section{Remarks}

Spination of patellae I, III is unusual: unlike all other species, a spine is present ventrally on a patella (I), and only in A. bluewater and A. undara are spines absent on patella III.

\section{Distribution and Habitat}

Known only from rainforest on the West Claudie River, Cape York, Queensland.

\section{Etymology}

A noun in apposition taken from the name of the type locality.

\section{Amauropelma gayundah Raven and Stumkat, sp. nov.}

Figures $4,13,27 f$, Table 5

\section{Material Examined}

\section{Holotype}

$\delta$, Gayundah Ck, Hinchinbrook Is, Queensland, Australia, $18^{\circ} 21^{\prime} \mathrm{S}, 146^{\circ} 13^{\prime} \mathrm{E}$, rainforest, pitfalls, 8-17 November 1984, V.Davies, J. Gallon (QM S32990).

\section{Paratype}

Australia: Queensland: $1 \delta$, same data as holotype but $10 \mathrm{~m}$ altitude, 7-14 November 1984 (QM S19813).

\section{Diagnosis}

Differs from other sighted species in that the median apophysis has no lumen viewed ventrally.

\section{Description}

Holotype male (QMS 32290)

Carapace 2.12 long, 1.60 wide. Abdomen 1.72 long, 1.08 wide.
Colour. Carapace and legs light yellow. Abdomen dorsally mottled brown (paratype) with 3-4 pairs of large white spots. Eyes. Lateral eyes on separate tubercles; from above ALE at back edge of AME; AME:ALE:PME:PLE, 6:6:5:5. Spines. As for genus except as noted. I: fe pv1p1d3; ti p1r1; me p1r1. II, as for I but fe pv1p1d3r1. III: fe pv1p2d3r2; pa r1; ti p2d3r2; me p1.1.2r1.2.2v2.2.2.4. IV: fe pv1p2d4r2; pa r1; ti p2d1r2v2.1.1.1.1; me p1.1.2r1.1.2v2.2.2.4. Palp: fe p1d2; pa 0; ti p2d1; cymbium with no subapical spines. Palp. Patellar fold normal, broken, forms two prominent conical processes. Tibial apophysis a single deeply divided bifid retrolateral process. Cymbium with small but distinct retrobasal lobe and strongly produced probasal lobe. Median apophysis without ventrally evident lumen.

\section{Female}

Unknown.

\section{Distribution and Habitat}

Known only from rainforest at Gayundah Creek, Hinchinbrook Island, north-eastern Queensland.

\section{Etymology}

A noun in apposition taken from the type locality.

Amauropelma gordon Raven and Stumkat, sp. nov. Figures 4, 14a-e, 15, 16, Table 6

\section{Material Examined}

Holotype

$\delta$, Gordon $\mathrm{Ck}$, Iron Range, Queensland, Australia, $12^{\circ} 43^{\prime} \mathrm{S}, 143^{\circ} 19^{\prime} \mathrm{E}$, pitfall, 28 June 1976, V. Davies, R. Raven (QM S31356).

Table 6 Leg measurements of Amauropelma gordon.

\begin{tabular}{lccccc}
\hline \multicolumn{7}{l}{ Holotype male (QMS31356). } \\
\hline & Leg 1 & Leg 2 & Leg 3 & Leg 4 & Palp \\
\hline Femur & 2.31 & 1.92 & 1.69 & 2.46 & 1.08 \\
Patella & 0.77 & 1.00 & 0.77 & 0.92 & 0.46 \\
Tibia & 2.07 & 1.46 & 1.31 & 1.92 & 0.54 \\
Metatarsus & 2.23 & 1.38 & 1.46 & 2.23 & \\
Tarsus & 1.08 & 0.69 & 0.69 & 1.00 & 0.92 \\
\hline Total & 8.46 & 6.45 & 5.92 & 8.53 & 3.00 \\
\hline
\end{tabular}

Allotype female (QMS31357).

\begin{tabular}{llllll}
\hline & Leg 1 & Leg 2 & Leg 3 & Leg 4 & Palp \\
\hline Femur & 2.00 & 1.92 & 1.85 & - & 0.69 \\
Patella & 1.08 & 0.92 & 0.92 & - & 0.38 \\
Tibia & 1.85 & 1.54 & 1.31 & - & 0.61 \\
Metatarsus & 1.46 & 1.38 & 1.38 & - & \\
Tarsus & 0.61 & 0.69 & 0.85 & - & 0.77 \\
\hline Total & 7.00 & 6.45 & 6.31 & 2.45 & \\
\hline
\end{tabular}



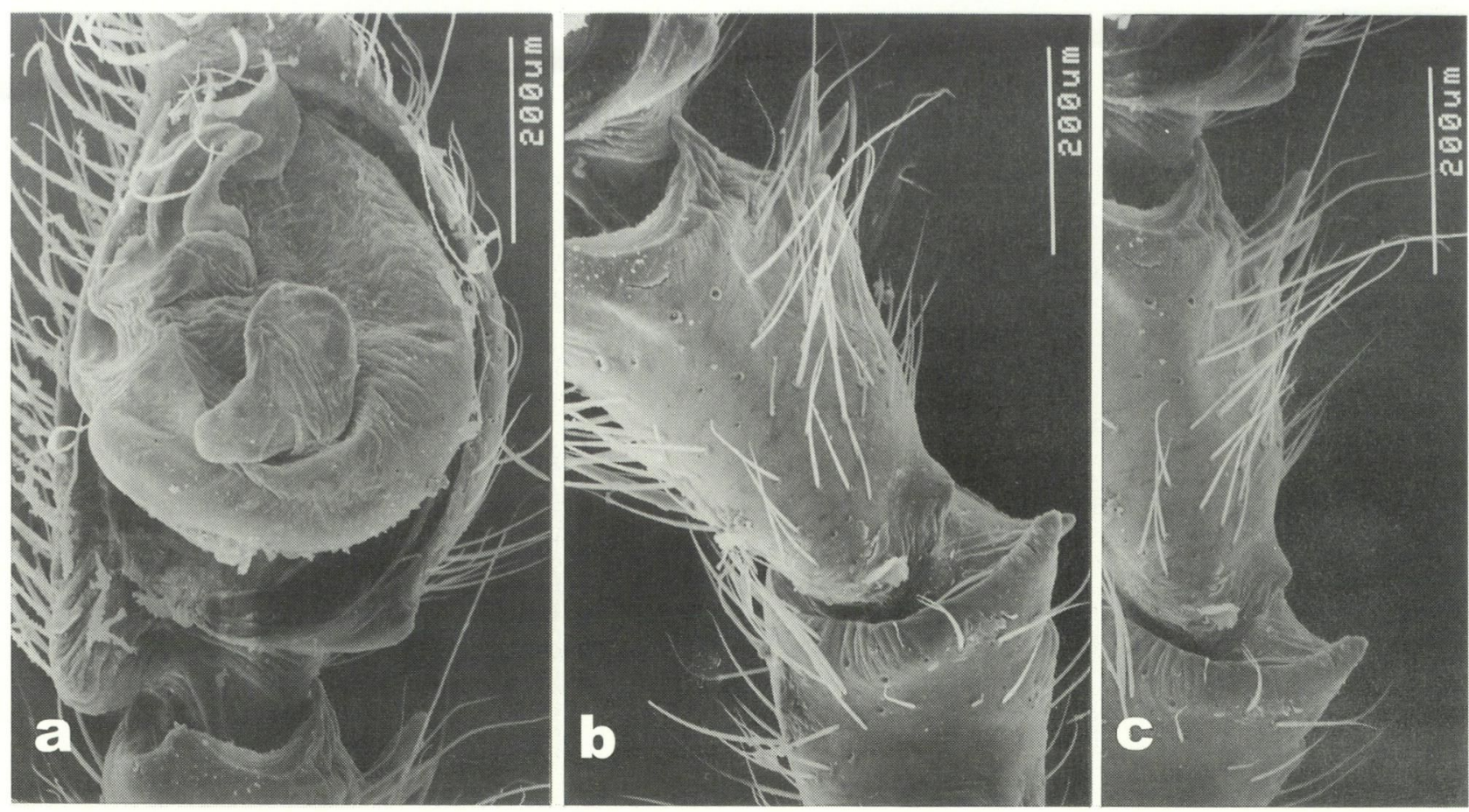

Figure 13 Amauropelma gayundah sp. nov.: male palp, ventral right, scanning electron micrographs: a, cymbium, bulb and distal tibia, ventral view; b, c, tibia and patella showing apophyses, retroventral view (b), ventral view (c).
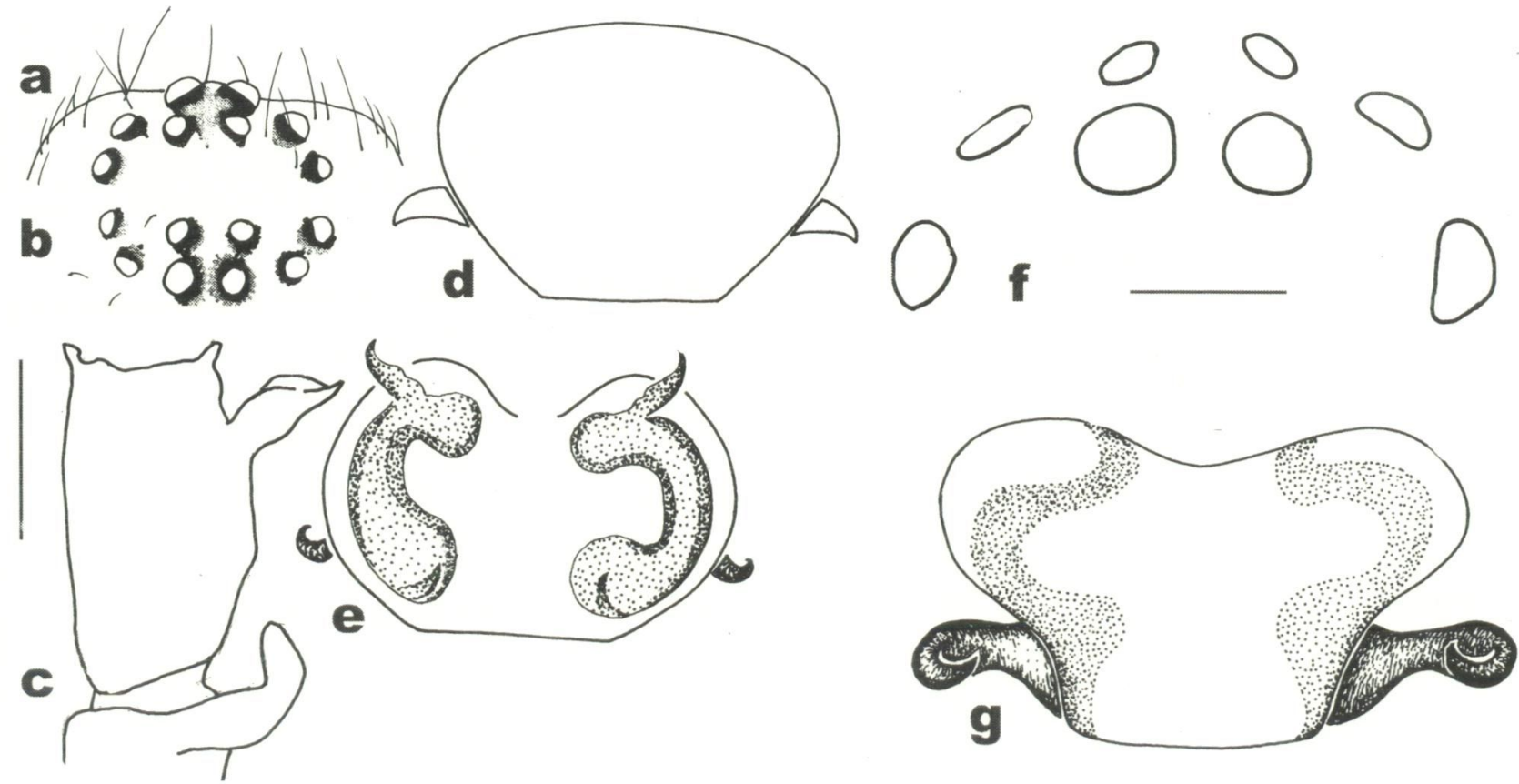

Figure 14 Amauropelma. a-e, A. gordon sp. nov.: holotype male (a-c), allotype female (d, e): a, b, eyes, dorsal view (a), frontal view (b); palpal patella and tibia, ventral view (c); d, e, epigyne, ventral view (d), dorsal view (e); f, $\mathrm{g}$, A. claudie sp. nov., $\mathrm{f}$, eyes, dorsal view; $\mathrm{g}$, epigyne, ventral view. Scale line $=2 \mathrm{~mm}$ for $\mathrm{a}, \mathrm{b}, 1 \mathrm{~mm}$ for $\mathrm{c}$, $0.5 \mathrm{~mm}$ for $\mathrm{d}, \mathrm{e}, 0.3 \mathrm{~mm}$ for $\mathrm{f}, \mathrm{g}$. 

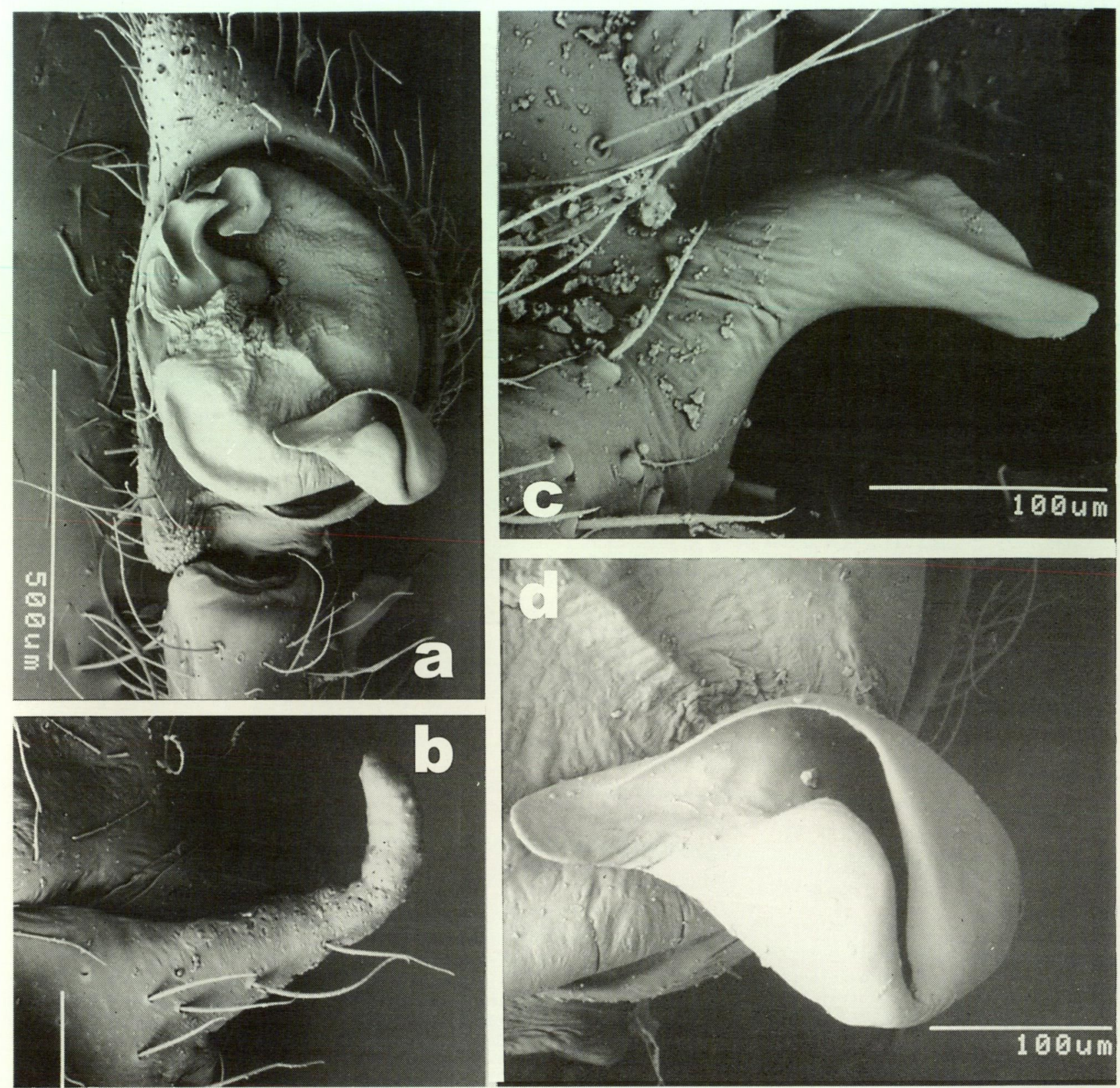

Figure 15 Amauropelma gordon sp. nov.: male palp, ventral right, scanning electron micrograph, ventral view: a, cymbium, bulb and distal tibia; b, patellar apophysis; c, tibial apophysis; (d) median apophysis; all in ventral view.

\section{Allotype}

Australia: Queensland: + , same data as holotype (QM S31357).

\section{Paratypes}

Australia: Queensland: $2 \delta$, same data as holotype (QM S31358).

\section{Diagnosis}

Males are readily distinguished from all other species by the long vermiform patellar process on the male palpal patella; females differ from those of A. claudie in the lateral horns being close to the plate.

\section{Description}

Holotype male (QM S31356)

Carapace 2.62 long, 2.00 wide. Abdomen 2.53, 1.69 wide.

Colour. Carapace orange with narrow shadows around eyes. Legs yellow without bands. Abdomen pallid. Eyes. AME:ALE:PME:PLE, 6:5:4:4, all small. Chelicerae. Promargin 2 teeth; retromargin 5 teeth. Spines. As for genus except as noted. I: fe pv1 p1d3r2; ti p1r1; me p1r1v2.2.2. II: fe pv1 p1d3r2; ti p1r1; me p1r1. III: fe pv1p2d3r3; pa p1r1; ti p2d3r2; me p5r2+2 (lower 2 strong). IV: fe p2d3r2; pa 0; ti p2d2r2; me p2+3l rp2+2l v2.2.1.2. Palp: fe p1d1.2; pa p1; ti p2d1. Spines on ventral and lower lateral 

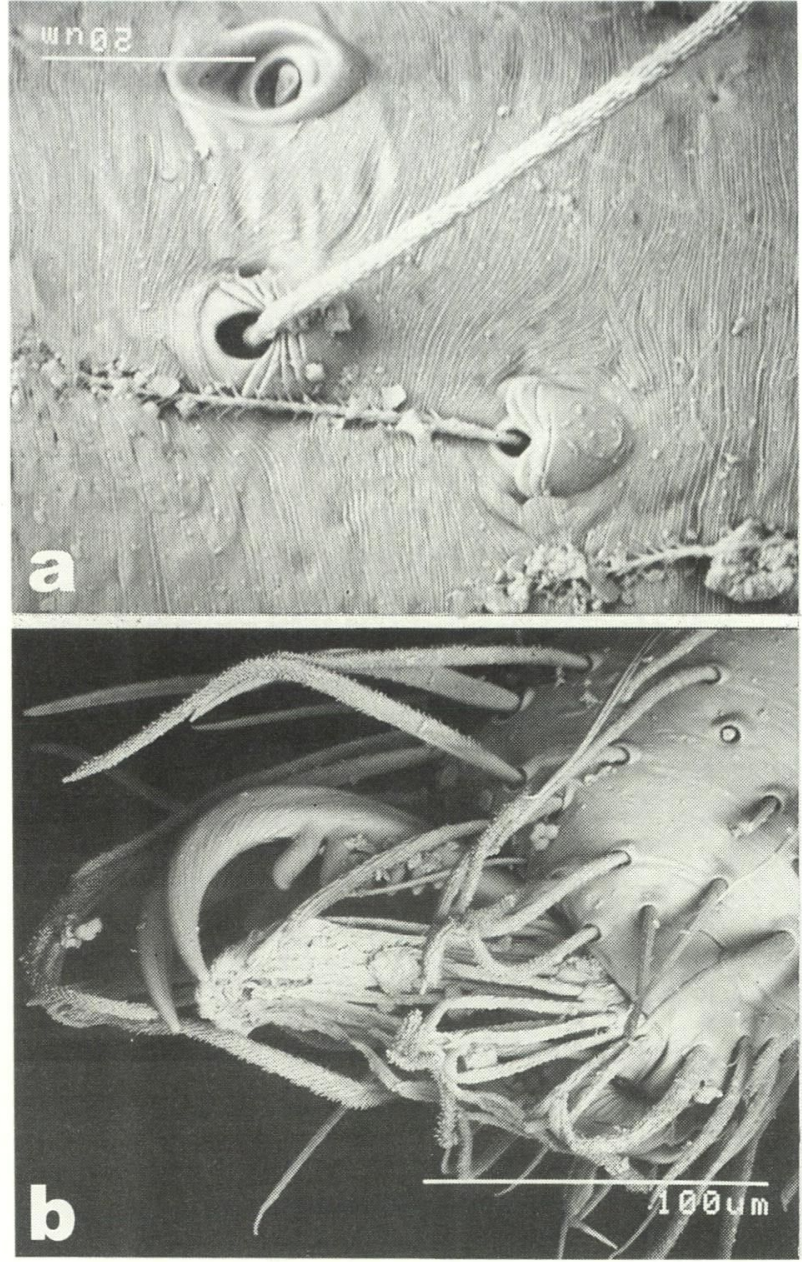

Figure 16 Amauropelma gordon sp. nov. Female, scanning electron micrographs: a, adpressed and erect trichobothria on tarsi; $b$, distal tarsus, paired claws and claw tufts, lateral view.

tibia and metatarsi, strong and very long on metatarsi, at least half length of segment, shorter on tibiae I, II. Spinnerets. ALS ca. 1 diameter apart, PMS as long but thinner than PLS. Palp. Patella ventral joint extended into long blunt curved process. Tibia with proventral thickening and small broad process widening and then tapering near tip to blunt point, two blunt distal collars on proventral face of tibia flank broad cymbial lobe. Tegulum forming a reflected G-shape; dark groove and curved process arising off prolateral tegulum and in front of that an elaborate short hooked grooved process with transluscent conductor flared dorsally. Median apophysis narrow, gourd-shaped with narrow ridge and hangs basally back over back edge of tegulum; lateral bulb very elevated. Cymbium retrobasally with slight flange forming basal ridge, no retrobasal lobe. Annuli on subtegulum; interlocking lobes evident.

\section{Allotype female (QM S31357)}

Carapace 2.62 long, 2.00 wide. Abdomen 2.84, 1.85 wide.
Colour. As for male. Eyes. AME:ALE:PME:PLE, 5:5:5:5. Spines. As for genus except as noted. I: fe pv1w p1d3r1. II: fe p1d3r1. III: fe p2d3r1; pa r1; ti p2d3r2; me p2+2l r2+2l v2.2.2. IV: fe p2d3r1; pa 0; ti p2d3r2v1.2.2; me p2+2l r2+3l v2.2.2. Palp: fe p1d1.2; pa p1; ti p2r2; ta p3r3. Epigyne. Externally broad, rounded plate without posterior projection; horns long, sinuous without obvious duct; anteriorly on (ventral) left a sclerotised horn (presumably matched by another still on female) which appears to be copulatory duct leading to small head of spermathecae and proceeding back under fold through thick walled duct to basal lobe.

\section{Distribution and Habitat}

Known only from lowland rainforest at Gordon Creek, Iron Range, Cape York Peninsula, Queensland.

\section{Etymology}

A noun in apposition taken from the name of the type locality.

\section{Amauropelma hasenpuschi \\ Raven and Stumkat, sp. nov. Figures 3f-g, 4, 17, 18, 33d, Table 7}

\section{Material Examined}

\section{Holotype}

o, South Johnstone Forestry Camp, $2 \mathrm{~km}$ E., Queensland, Australia, 17 $36^{\circ} \mathrm{S}, 1^{\circ} 6^{\circ} 00^{\prime} \mathrm{E}$, rainforest, pitfall, 1 December 1993-25 February 1994, J. Hasenpusch (QM S31362).

\section{Allotype}

Australia: Queensland: $q$, South Johnstone Forestry Camp, $2 \mathrm{~km} \mathrm{E.,} 17^{\circ} 36^{\prime} \mathrm{S}, 146^{\circ} 00^{\prime} \mathrm{E}$, rainforest, pitfall, 1 December 1993-25 February 1994, J. Hasenpusch (QM S31363).

\section{Paratypes}

Australia: Queensland: $2 \delta$, Copperlode Dam Rd (NQ 42), $16^{\circ} 58^{\prime} \mathrm{S}, 145^{\circ} 42^{\prime} \mathrm{E}$, rainforest, pitfall, 27 October 1991-23 July 1992, P. Lawless, R. Raven, M. Shaw (QM S32910); 1 đ。, Graham Ra, 17²17'S, $145^{\circ} 57^{\prime} \mathrm{E}$, rainforest, pitfall, 1 November-8 December 1995, G. Monteith, G. Thompson, D. Cook (QM S32915); 1 ð, Hughes Rd, Topaz, 17²6'S, $145^{\circ} 42$ 'E, pitfall, July-September 1993, G. Monteith, S. Breeden (QM S32911); 1 , Koombooloomba Dam, 4 km S., $17^{\circ} 50^{\prime} \mathrm{S}, 145^{\circ} 36^{\prime} \mathrm{E}$, rainforest, pitfall, 9 December 1989-5 January 1990, G. Monteith, G. Thompson, H. Janetzki, (QM S18897); 1 q, Maalan SF on $\mathrm{H}^{\prime}$ way, $17^{\circ} 35^{\prime} \mathrm{S}, 145^{\circ} 35^{\prime} \mathrm{E}$, rainforest, pitfall, 10 January-31 March 1995, G. Monteith, J. Hasenpusch (QM S32898); 1 đे, Massey Ck (BS3), $17^{\circ} 37^{\prime} \mathrm{S}, 145^{\circ} 33^{\prime} \mathrm{E}$, rainforest, pitfall, 2-30 November 


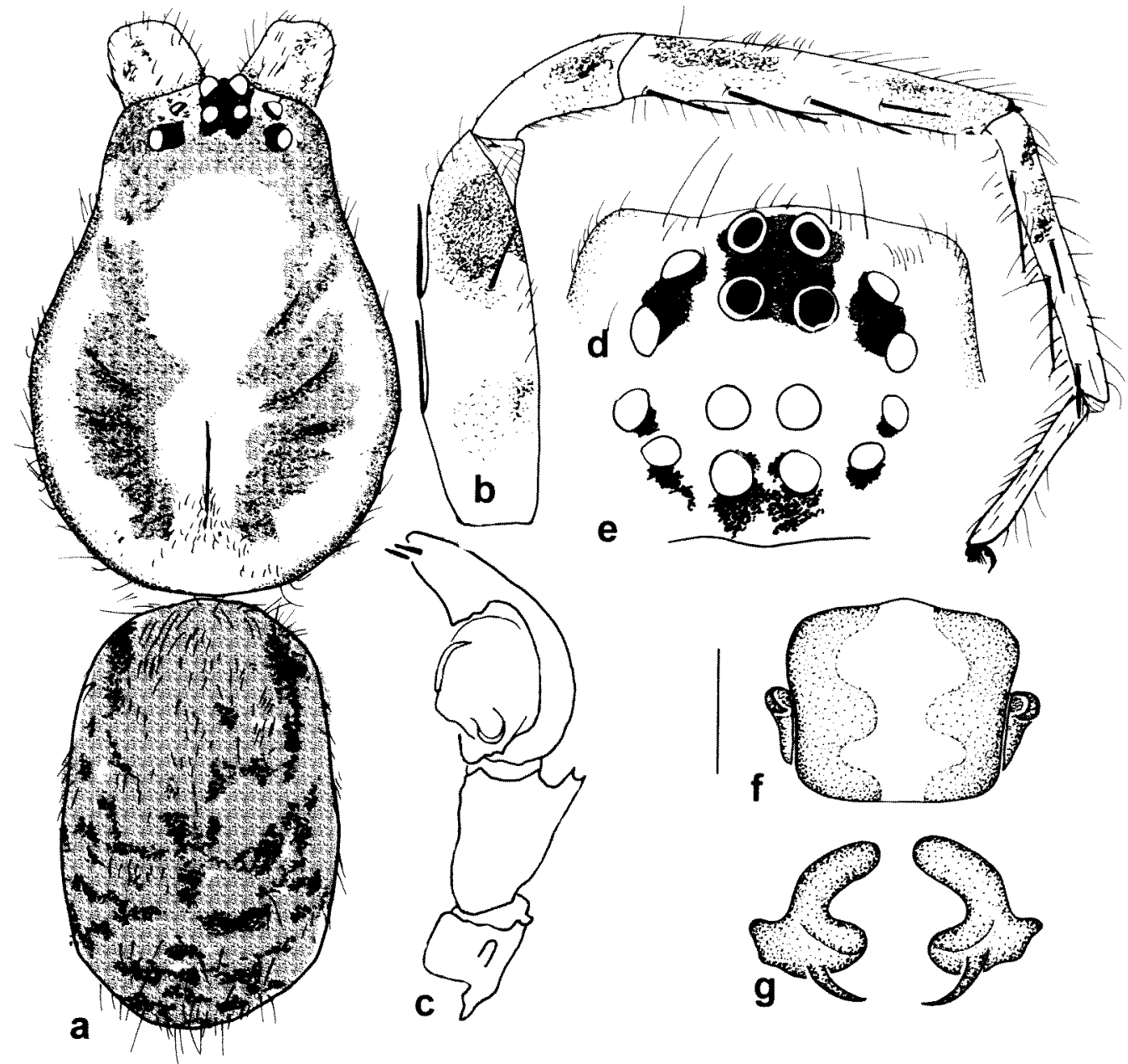

Figure 17 Amauropelma hasenpuschi sp. nov.: holotype male (a-e), allotype female (f, $\mathrm{g}$ ): a, carapace, chelicerae and abdomen, dorsal view; $b$, leg I, prolateral view; $c$, palpal patella to cymbium, retroventral view showing patella and tibial apophyses; d, e, eyes, dorsal view (d), front view (e); f, g, epigyne, ventral view (f), dorsal view (g). Scale line $=2 \mathrm{~mm}$ for $a, b ; 1 \mathrm{~mm}$ for $c-e ; 0.3 \mathrm{~mm}$ for $\mathrm{f}, \mathrm{g}$.

1995, L. Umback (QM S39119); 1 ठ 1 1 \&, Palmerston $\mathrm{NP}, 17^{\circ} 35^{\prime} \mathrm{S}, 145^{\circ} 41^{\prime} \mathrm{E}$, rainforest, pitfall, $25 \mathrm{July}-30$ November 1992, R. Raven, P. Lawless, E. Lawless, M. Shaw (QM S21935, AMNH); 1 o,, Polly Ck (Hasenpusch property), $17^{\circ} 28^{\prime} \mathrm{S}, 146^{\circ} 00^{\prime} \mathrm{E}$, rainforest, intercept flight trap, 7 March-15 May 1995, G. Monteith, J. Hasenpusch (QM S41788); 1 o, Stone $\mathrm{Ck}$ (Hasenpusch), $17^{\circ} 18^{\prime} \mathrm{S}, 146^{\circ} 00^{\prime} \mathrm{E}$, intercept flight trap, 1 October-1 November 1995, J. Hasenpusch (QM S32912); 1 o, Tower near The Crater NP, $17^{\circ} 27^{\prime} \mathrm{S}, 145^{\circ} 28^{\prime} \mathrm{E}$, rainforest, pitfall, 7 March-15 May 1995, G. Monteith, J. Hasenpusch (QM S41786); 1 o, Tower near The Crater NP, $17^{\circ} 27^{\prime} \mathrm{S}, 145^{\circ} 28^{\prime} \mathrm{E}$, flight intercept trap, 25 November 1994-10 January 1995, G. Monteith, J. Hasenpusch (QM S31365); 3 o', Wongabel SF, $17^{\circ} 19^{\prime} \mathrm{S}, 145^{\circ} 29^{\prime} \mathrm{E}$, rainforest, pitfall, 23 July-26 November 1992, R. Raven, P. Lawless, E. Lawless, M. Shaw (QM S22616, WAM T42601, AMNH).

\section{Other Material}

Australia: Queensland: $1 \delta$, Mt Fisher, Kjellberg $\mathrm{Rd}, 17^{\circ} 31^{\prime} \mathrm{S}, 145^{\circ} 33^{\prime} \mathrm{E}$, rainforest, pitfall, 1 December
Table 7 Leg measurements of Amauropelma hasenpuschi.

Holotype male (QMS31362).

\begin{tabular}{llllll}
\hline & Leg 1 & Leg 2 & Leg 3 & Leg 4 & Palp \\
\hline Femur & 2.61 & 2.31 & 2.15 & 2.85 & 0.92 \\
Patella & 1.31 & 1.08 & 0.96 & 1.15 & 0.46 \\
Tibia & 2.38 & 1.85 & 1.54 & 2.46 & 0.46 \\
Metatarsus & 1.92 & 1.77 & 2.00 & 2.77 & \\
Tarsus & 1.15 & 1.08 & 1.23 & 1.77 & 1.08 \\
\hline Total & 9.37 & 8.09 & 7.88 & 11.00 & 2.92 \\
\hline
\end{tabular}

Allotype female (QMS31363).

\begin{tabular}{lccccc}
\hline & Leg 1 & Leg 2 & Leg 3 & Leg 4 & Palp \\
\hline Femur & 2.38 & 1.84 & 1.92 & 2.54 & 1.00 \\
Patella & 1.23 & 1.08 & 0.92 & 1.00 & 0.46 \\
Tibia & 1.85 & 1.38 & 1.31 & 2.23 & 0.69 \\
Metatarsus & 1.42 & 1.38 & 1.61 & 2.38 & \\
Tarsus & 0.85 & 0.85 & 1.08 & 1.54 & 0.69 \\
\hline Total & 7.73 & 6.53 & 6.84 & 9.69 & 2.84 \\
\hline
\end{tabular}



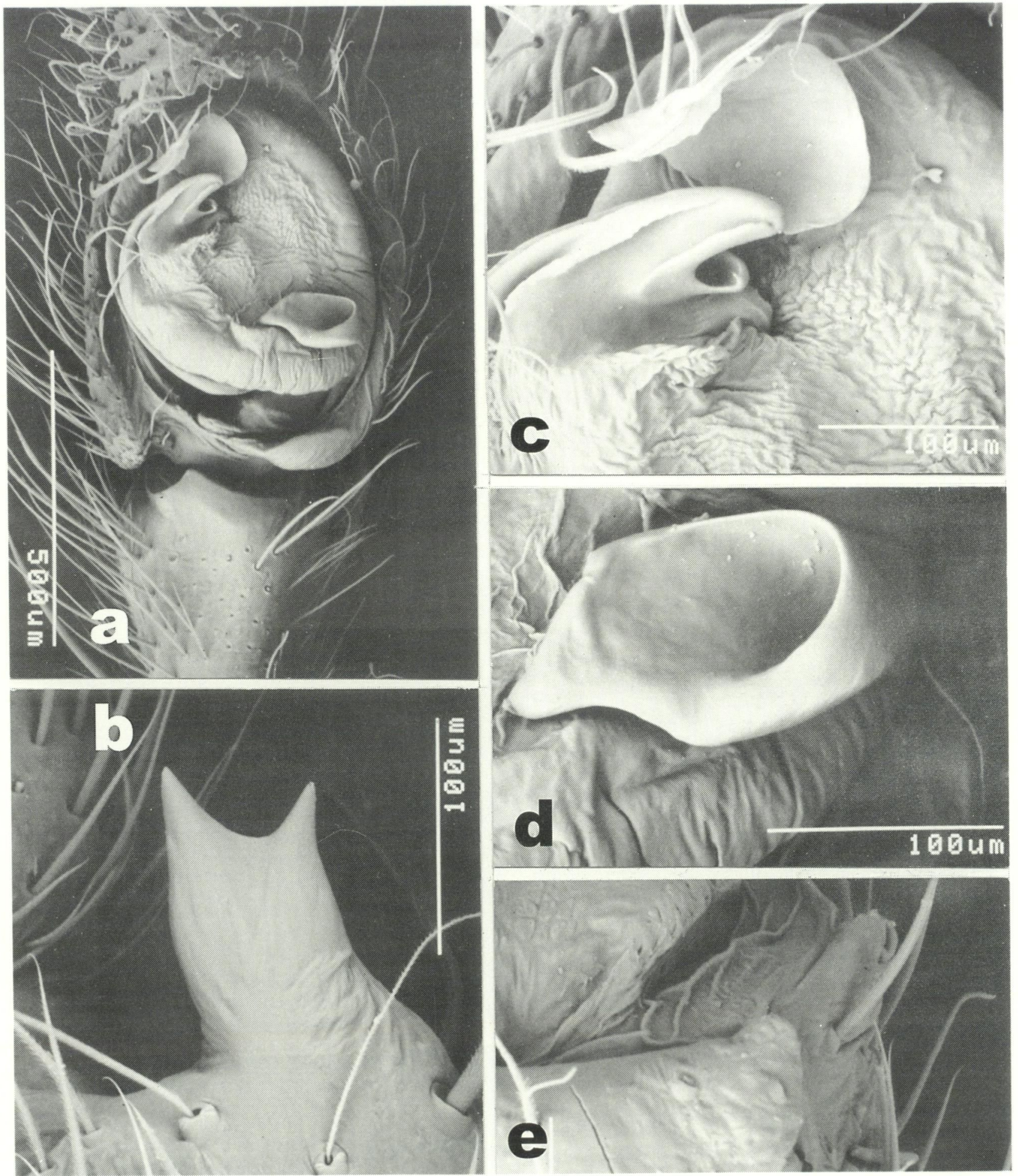

Figure 18 Amauropelma hasenpuschi sp. nov.: male palp, ventral right, scanning electron micrographs: a, cymbium, bulb and distal tibia, ventral view; b, tibial apophysis, rotated ventral view; c, embolic plate and conductor, ventral view; $d$, median apophysis, ventral view; e, patellar apophyses, ventral view.

1993-25 February 1994, J. Hasenpusch (QM S32897).

\section{Diagnosis}

Differs from most other species in the subquadrate shape of the epigynal plate and from A. bluewater in the relatively larger lateral horns and the rectanguloid median apophysis with very short 'handle'.

\section{Description}

Holotype male (QM S31362)

Carapace 3.23 long, 2.46 wide. Abdomen 2.61, 
1.62 wide.

Colour. Carapace light brown with dark bands midlaterally and narrow black marginal band. Chelicerae deep reddish brown; legs orange brown with incomplete dark bands: femora, mid- and distal; patellae distal; tibiae mid- and subdistal. Abdomen dorsally pallid with 3 inverted $U$ dark crescents in posterior half and laterally mottled, ventrally pallid with one pair few light shadows medially. Iridescent green sheen on legs. Eyes. AME:ALE:PME:PLE, 9:8:7:10. On low tubercles. ALE set along front edge of PME. Chelicerae. Promargin with 2 small teeth; retromargin with 4 spaced large teeth and 1 minute basally. Sternum. Margins rounded. Spines. As for genus except as noted. I: fe pv1p1 d3r1; me p1. II: fe pv1p1 d3r1; pa 0; ti p1; me p1. III: fe pv1p2d3r3; pa p1r1; ti p2d3r2; me p2+2l r2+12. IV: fe pv1p2d3r2; pa r1; ti p2d2r2; me p2+2l r2+2l v2.2.2.2. Palp: fe p1d1.2; pa 0 ; ti p2d1; cymbium with 2 subapical spines. Claws. Tufts small, dense; not as long as in $9 ; 3$ teeth on I, 1-2 on IV, and basal onychium evident on tarsi. Legs. Trochanteral notches deep. Palp. Patellar fold normal, broken, small protrusive lip on retrolateral corner. Tibia with retroventral distal widening bearing short, deeply and equally bipartite apophysis; distal tibia with broad triangular extension proventrally and prodorsally (the larger) flanking broad bluntly rounded probasal cymbial process; retrolateral and distal cymbial inner edge a low to high (basally) flange. Subtegulum locking process a blunt cone, tegular interface barely indented. Median apophysis an open gourd-shaped scoop set just inside O-shaped tegulum. Broad, flared conductor near embolic division and begins as circular opening. Embolic plate distally divided into two apices, distal larger, proximal slender. Spinnerets. Apices inverted; ALS ca. 1 diameter apart and twice diameter of PLS.

\section{Allotype female (QM S31363)}

Carapace 3.23 long, 2.42 wide. Abdomen 4.00, 3.08 wide.

Colour. Carapace and abdomen almost without pattern; legs banded as in 0 . Eyes. AME:ALE:PME: PLE, 9:7:8:7. Chelicerae. Promargin with 2 teeth; retromargin with 5 . Spines. As for genus except as noted. I: fe pv1 strong p1d3. II: fe p1d3. III: fe pv1w p2d3r2; pa p1r1; ti p2d3r2; me p2+2l (lower) $\mathrm{r} 2+21$ (lower) v1.2.2.2. IV: fe p2d3 r1; pa 0 ; ti p2d3 r2; me p2+2l r2+2l v2.2.2. Palp: fe p1d2; pa 0 ti p1d3; ta p3. Claws. Long thin with 3-6 (I, II) or 1 (III, IV) teeth medially. Tufts small distinct on sessile process I, II or raised plate III, IV. All tarsi including palp with basal mound at $1 / 5$. Spinnerets. Inverted but ALS separated by 1 diameter, PMS thicker than PLS. Epigyne. Externally almost square rounded plate with a rounded vertical horn laterally. Horns presumably receive median apophysis as they are clearly not ducts but sclerotised guides. Copulatory duct beside horns lead forward to enlarged head and back laterally to enlarged basal receptaculum with long entally directed fertilisation ducts.

\section{Distribution and Habitat}

Known only from rainforest at in the central portion of the Wet Tropics Heritage Area, northeastern Queensland.

\section{Variation}

The tibial apophysis of males from different localities shows slight variation in the relative sizes of the apical prongs but otherwise the morphology is acceptably conspecific.

\section{Etymology}

For Mr Jack Hasenpusch, the collector of some of the types.

Amauropelma leo Raven and Stumkat, sp. nov. Figures 4, 19, Table 8

\section{Material Examined}

\section{Holotype}

+ , Leo's Ck, Coen, Queensland, Australia, $13^{\circ} 32^{\prime} \mathrm{S}, 143^{\circ} 27^{\prime} \mathrm{E}, 25$ July 1976, P. Filewood (QM S32903).

\section{Diagnosis}

Differs from all other species in the very reduced sizes of the ALE, PME and PLE.

\section{Description}

\section{Holotype female (QM S32903)}

Carapace 2.32 long, 1.72 wide. Abdomen 2.20 long, 1.40 wide. Total length 5 .

Colour. Carapace and legs orange brown, chelicerae darker; abdomen fawn without pattern. Carapace. Long fovea extends as far posterior of posterior declivity as anterior. Eyes. Small; $\mathrm{AME}>\mathrm{PME}=\mathrm{PLE}=\mathrm{ALE}$. 3 rows, 2.4.2, second row slightly procurved. From above, front of ALE set

Table 8 Leg measurements of Amauropelma leo.

Holotype female (QM S32901).

\begin{tabular}{llllll}
\hline & Leg 1 & Leg 2 & Leg 3 & Leg 4 & Palp \\
\hline Femur & 2.69 & 2.56 & 2.38 & 3.00 & 1.31 \\
Patella & 1.56 & 1.44 & 1.25 & 1.44 & 0.50 \\
Tibia & 2.38 & 2.06 & 1.63 & 2.50 & 0.63 \\
Metatarsus & 1.88 & 1.69 & 1.88 & 2.75 & \\
Tarsus & 0.75 & 0.81 & 1.25 & 1.25 & 0.88 \\
\hline Total & 9.26 & 8.56 & 8.14 & 10.94 & 3.32 \\
\hline
\end{tabular}




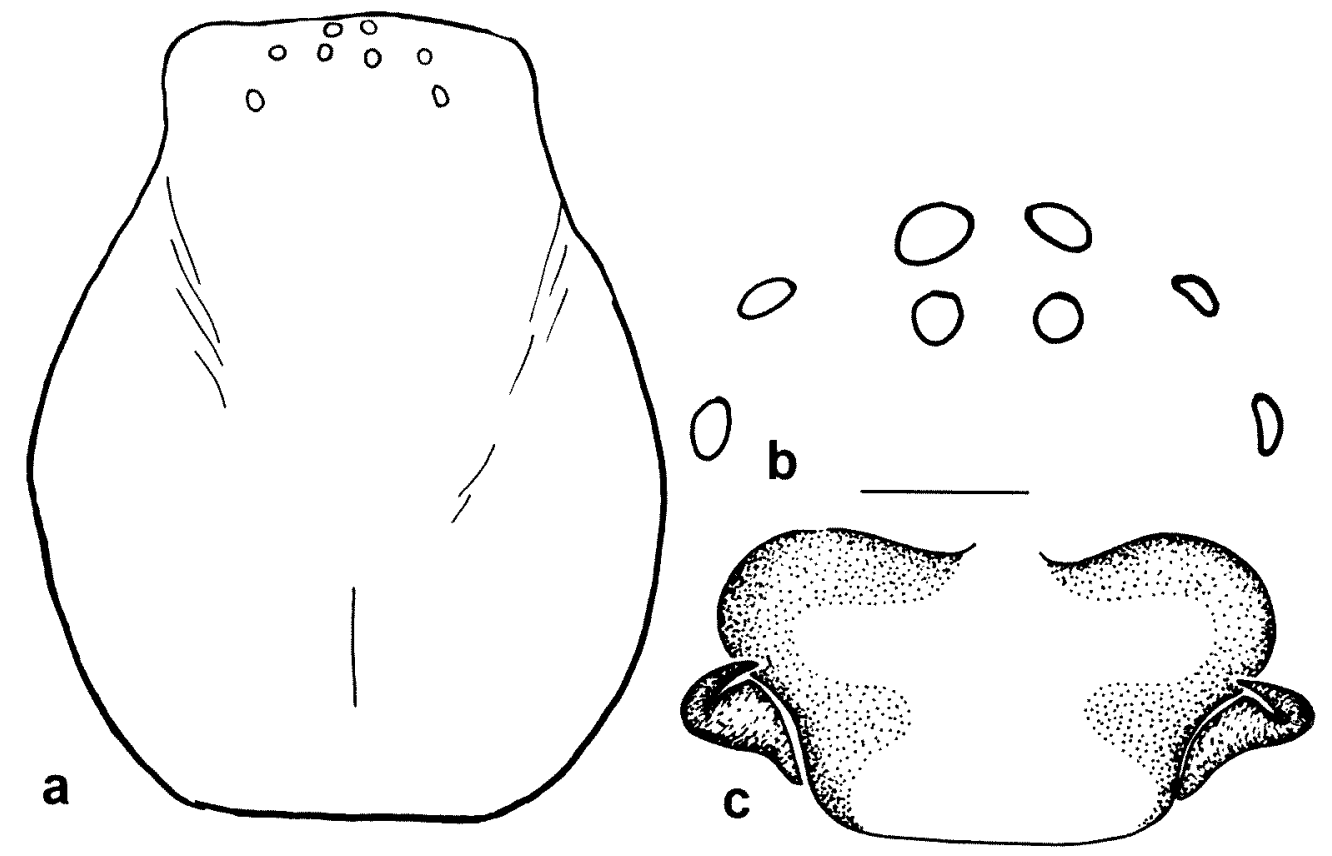

Figure 19 Amauropelma leo sp. nov., holotype female. a, carapace, dorsal view; b, eyes, dorsal view; c, epigyne, ventral view. Scale lines $=0.9 \mathrm{~mm}$ for $a, 0.3 \mathrm{~mm}$ for $b, 0.2 \mathrm{~mm}$ for $c$.

just anterior to front of PME; ALE clearly closer to PLE than ALE; PLE clearly in separate row to PME. MOQ narrower behind than in front; from front ALE set above AME but part below and part beside PME. Eye group $=0.55$ of head $/$ width; $\mathrm{PME}=6 \%$ of head width. Chelicerae. 2 teeth evident on promargin; 5 large and one small basal teeth on retromargin. Legs. Tarsal organ in distal third on I, basal 1/6 on III, IV. No scopula. Spines. As for genus except as noted. I: fe pv1p1d3. Rest as for $A$. mcilwraith except: II: fe pv1p2d3r1. III: fe p2d3r3. IV: fe p3d3r2. Palp: fe p1d1.2r1; pa p1; ti p2d2; ta p2.1r1.1. Epigyne. A broad plate with well rounded wings and soft curving lateral horns.

Male

Unknown.

\section{Distribution and Habitat}

Known only from rainforest at Leo Creek, near Coen, Cape York Peninsula.

\section{Etymology}

A noun in apposition taken from the type locality.

\section{Amauropelma mcilwraith}

Raven and Stumkat, sp. nov.

Figures 3e, 4, 20, 27c, Table 9

\section{Material Examined}

\section{Holotype}

o. $11 \mathrm{~km} \mathrm{W.} \mathrm{by} \mathrm{N}$. of Bald Hill, Mcllwraith
Range, Queensland, Australia, $13^{\circ} 44^{\prime} \mathrm{S}, 143^{\circ} 20^{\prime} \mathrm{E}$, $520 \mathrm{~m}, 27$ June-12 July 1989, T.A. Weir (ANIC).

\section{Diagnosis}

Differs from all other species in the extensive unsclerotised area surrounding the median apophysis.

\section{Description}

\section{Holotype male (ANIC)}

Carapace 2.68 long, 2.12 wide. Abdomen 2.20 long, 1.40 wide. Total length 4.8 .

Colour. Carapace yellow brown with light marginal and subcentral shadows. Abdomen dorsally pallid with many small dark spots forming no discernible pattern. Legs yellow brown with dark shadows on distal femora, lateral patellae and proximal and distal tibiae and metatarsi, annuli more evident on III, IV, than on I, II. Eyes. $\mathrm{AME}=\mathrm{ALE}<\mathrm{PME}=\mathrm{PLE}$. ALE oval; from front, lower

Table 9 Leg measurements of Amauropelma moilwraith.

\begin{tabular}{lccccc}
\hline \multicolumn{7}{l}{ Holotype male, ANIC. } \\
\hline & Leg 1 & Leg 2 & Leg 3 & Leg 4 & Palp \\
\hline Femur & 2.04 & 1.92 & 1.76 & 2.40 & 1.00 \\
Patella & 1.04 & 0.96 & 0.80 & 1.00 & 0.52 \\
Tibia & 1.88 & 1.40 & 1.32 & 2.00 & 0.52 \\
Metatarsus & 1.40 & 1.32 & 1.48 & 2.32 & \\
Tarsus & 0.88 & 0.72 & 1.20 & 1.20 & 1.04 \\
\hline Total & 7.24 & 6.32 & 6.20 & 8.92 & 3.08 \\
\hline
\end{tabular}



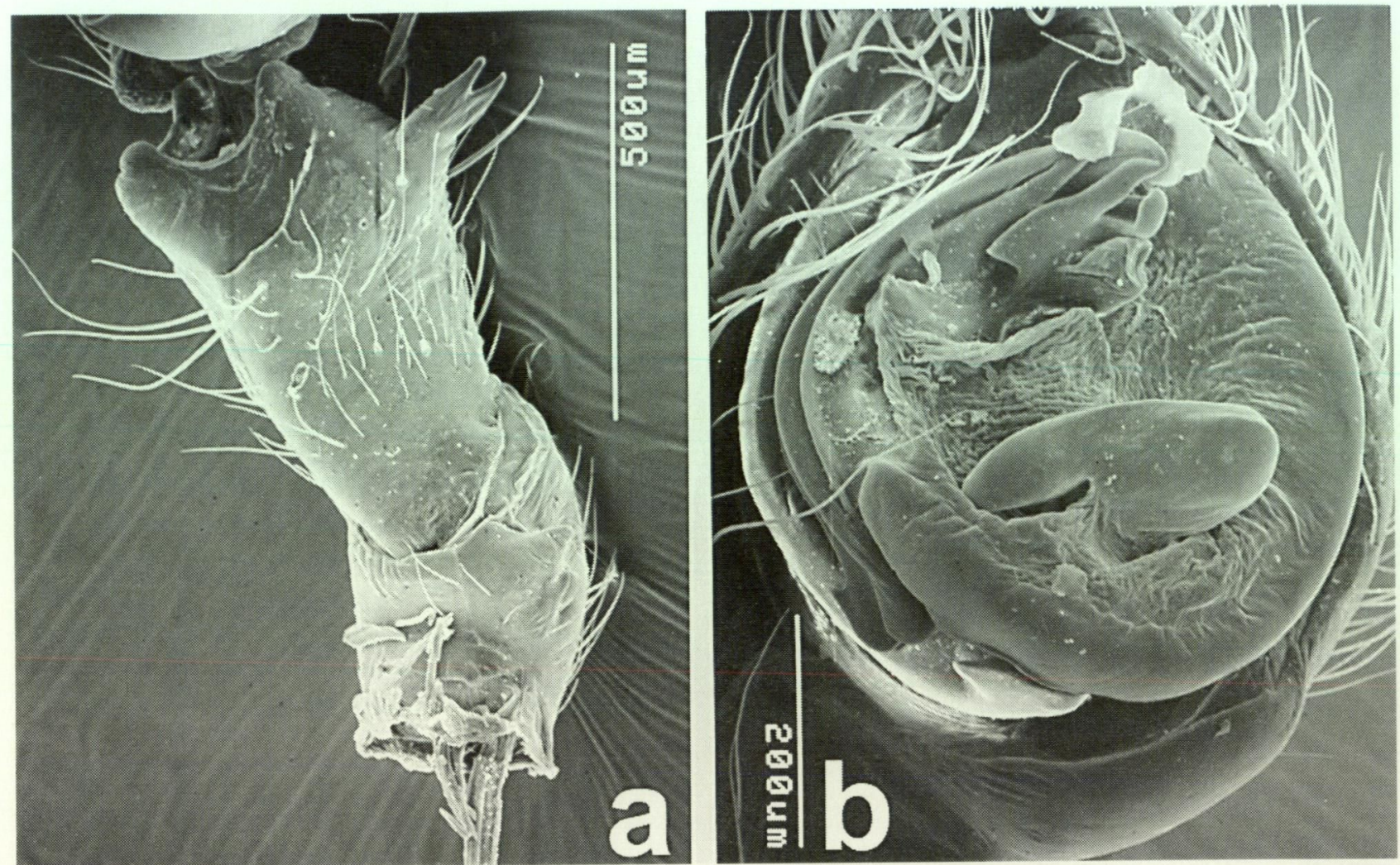

Figure 20 Amauropelma mcilwraith sp. nov.: male palp, ventral right, scanning electron micrographs: a, tibia and patella showing apophyses, retroventral view; $b$, cymbium and bulb, ventral view.

edge of ALE set just below upper edge of AME; from above, front of ALE in line with that of PME; ALE clearly closer to PLE than AME. Back eyes almost form 2 rows. Eye group $=0.67$ of head width; PME $=11 \%$ of head width. Legs. No scopula. Low tarsal organ in distal $1 / 3$ (I), to midpoint (II), or in basal 1/8/-1/6 (III, IV). Spines. As for genus except as noted. I, II: fe pv1p1d3; ti p1r1; me p1r1. III, IV: fe pv1p2d3r2; pa r1; ti p2d3r2; me p1.1.2r1.1.2v2.2.2. Palp: fe d1.2; pa 0; ti p2r1. Palp. Patella with large low retroventral process and more dorsal process indistinct. Tibia distally incrassate with transverse basoventral saddle, retrolateral apophysis on low mound with bifid tip. Cymbium deep with slightly sinuous prolateral margin; probasal process angular; retrobasal corner without process; apically conical without scopula. Bulb with tegulum forming narrow $\mathrm{C}$-shaped collar around broad unsclerotised area; median apophysis transverse with large lumen opening distally, 'handle' short. Embolic plate short, deeply folded but with basal sclerotised bar; large filmy folded conductor.

\section{Distribution and Habitat}

Known only from rainforest at McIlwraith Range, Cape York, Queensland.

\section{Etymology}

A noun in apposition taken from the type locality.

\section{Amauropelma monteithi}

Raven and Stumkat, sp. nov. Figures 4, 21-23, Table 10

\section{Material Examined}

\section{Holotype}

ठ․ Cape Tribulation, $2 \mathrm{~km} \mathrm{~W}$. (Site 4), Queensland, Australia, $16^{\circ} 04^{\prime} \mathrm{S}, 145^{\circ} 27^{\prime} \mathrm{E}$, rainforest, baited pitfall, 5-9 January 1983, G. Monteith (QM S31354).

\section{Allotype}

Australia: Queensland: $q$, Pilgrim Sands (NQ $25), 16^{\circ} 04^{\prime} \mathrm{S}, 145^{\circ} 27^{\prime} \mathrm{E}$, rainforest, pitfall, 5 November 1991-20 July 1992, R. Raven, P. Lawless, M. Shaw (QM S24866).

\section{Diagnosis}

Differs from other species in having a tarsal rod basally on leg tarsi.

\section{Description}

\section{Holotype male (QM S31354)}

Carapace 3.23 long, 2.54 wide. Abdomen 2.30, 1.54 wide.

Colour. Carapace and legs yellowish, darker shadow on striae and reticulations around eyes. Abdomen light grey over yellow brown, anteriorly 


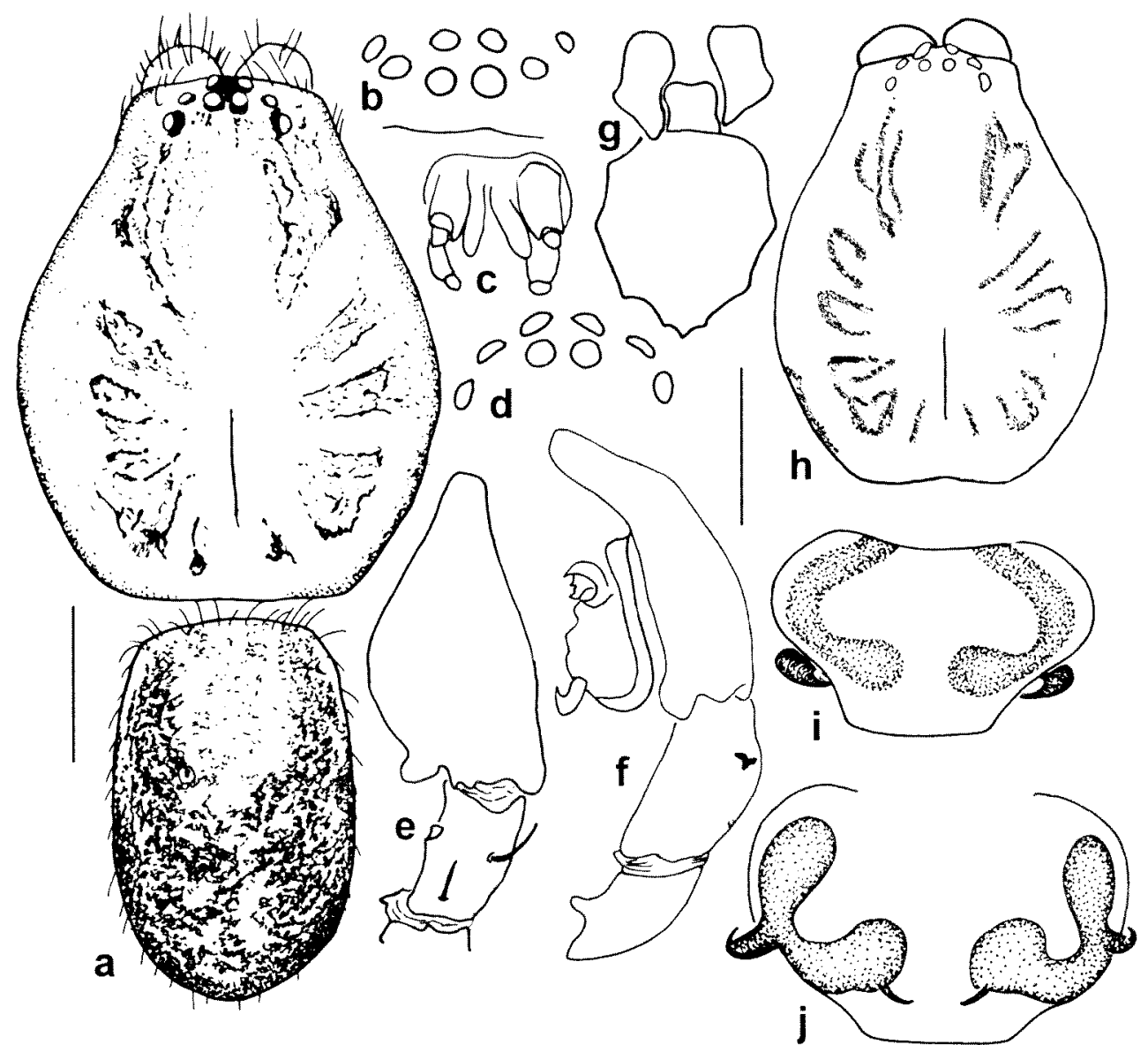

Figure 21 Amauropelma monteithi sp. nov.: holotype male $(\mathrm{a}, \mathrm{b}, \mathrm{d}-\mathrm{f})$, allotype female $(\mathrm{c}, \mathrm{h}-\mathrm{j})$ : $\mathrm{a}$, carapace, chelicerae and abdomen, dorsal view; b, d, eyes, dorsal view (d), front view (b); $c$, spinnerets, ventral view; e, $f$, palpal patella to cymbium, dorsal view (e), retrolateral view showing patella and tibial apophyses (f); $g$, sternum, maxillae and labium; h, carapace and chelicerae, dorsal view; i, j, epigyne, ventral view (i), dorsal view (j). Scale line $=1 \mathrm{~mm}$ for $\mathrm{a}, \mathrm{g}, \mathrm{h} ; 0.5 \mathrm{~mm}$ for $b-f ; 0.35 \mathrm{~mm}$ for $\mathrm{i}, \mathrm{j}$.

pallid, 3 irregularly paired pale areas posteriorly. Carapace. Pear-shaped; fovea $c a$. as long as midcarapace width, extends back to slope. Clypeus very narrow < AME. Fine iridescent sheen on carapace and lateral femora, no hairs evident. Anterior constriction very gradual, eyes confined to anterior half. Light cover of fine brown hairs dorsally and on margins; eye region domed downward from about back of anterior constriction. Eyes. AME:ALE:PME:PLE, 8:7:6:6. Two very recurved rows. AME on common tubercle and look to front, side and up. ALE look to front and side, PLE to side, PME up and front. Limited dark pigment around all but AME; AME project forward over clypeus. ALE set beside PME. All eyes of similar size and colour. Chelicerae. Promargin 3 small basal teeth; retromargin 4 small but larger teeth. Porrect; fang moderately long, boss distinct, triangular. Labium. Longer than wide, anterior margin thickened. Maxillae. Longer than wide, cylindrical. Sternum. Shield-shaped with cuticular extensions at midcoxae. Legs. Cuticle translucent. Scopula absent. Pilosity generally
Table 10 Leg measurements of Amauropelma monteithi.

\begin{tabular}{lcllll}
\hline \multicolumn{7}{l}{ Holotype male (QMS31354). } \\
\hline \multicolumn{7}{l}{ Leg 1 } & Leg 2 & Leg 3 & Leg 4 & Palp \\
\hline Femur & 2.61 & - & 2.31 & 3.23 & 1.31 \\
Patella & 1.31 & - & 1.00 & 1.38 & 0.54 \\
Tibia & 2.69 & - & 1.85 & 1.85 & 0.54 \\
Metatarsus & 2.23 & - & 2.00 & 3.23 & \\
Tarsus & 1.31 & - & 1.08 & 1.77 & 1.00 \\
\hline Total & 10.15 & 8.24 & 11.46 & 3.39 & \\
\hline
\end{tabular}

Allotype female (QMS24866).

\begin{tabular}{lccccc}
\hline & Leg 1 & Leg 2 & Leg 3 & Leg 4 & Palp \\
\hline Femur & 2.23 & 1.85 & 1.69 & 2.31 & 1.00 \\
Patella & 1.15 & 0.85 & 0.77 & 0.92 & 0.46 \\
Tibia & 1.85 & 1.46 & 1.38 & 2.23 & 0.54 \\
Metatarsus & 1.38 & 1.31 & 1.38 & 2.23 & \\
Tarsus & 0.85 & 0.69 & 0.92 & 1.46 & 0.61 \\
\hline Total & 7.46 & 6.16 & 6.14 & 9.15 & 2.61 \\
\hline
\end{tabular}



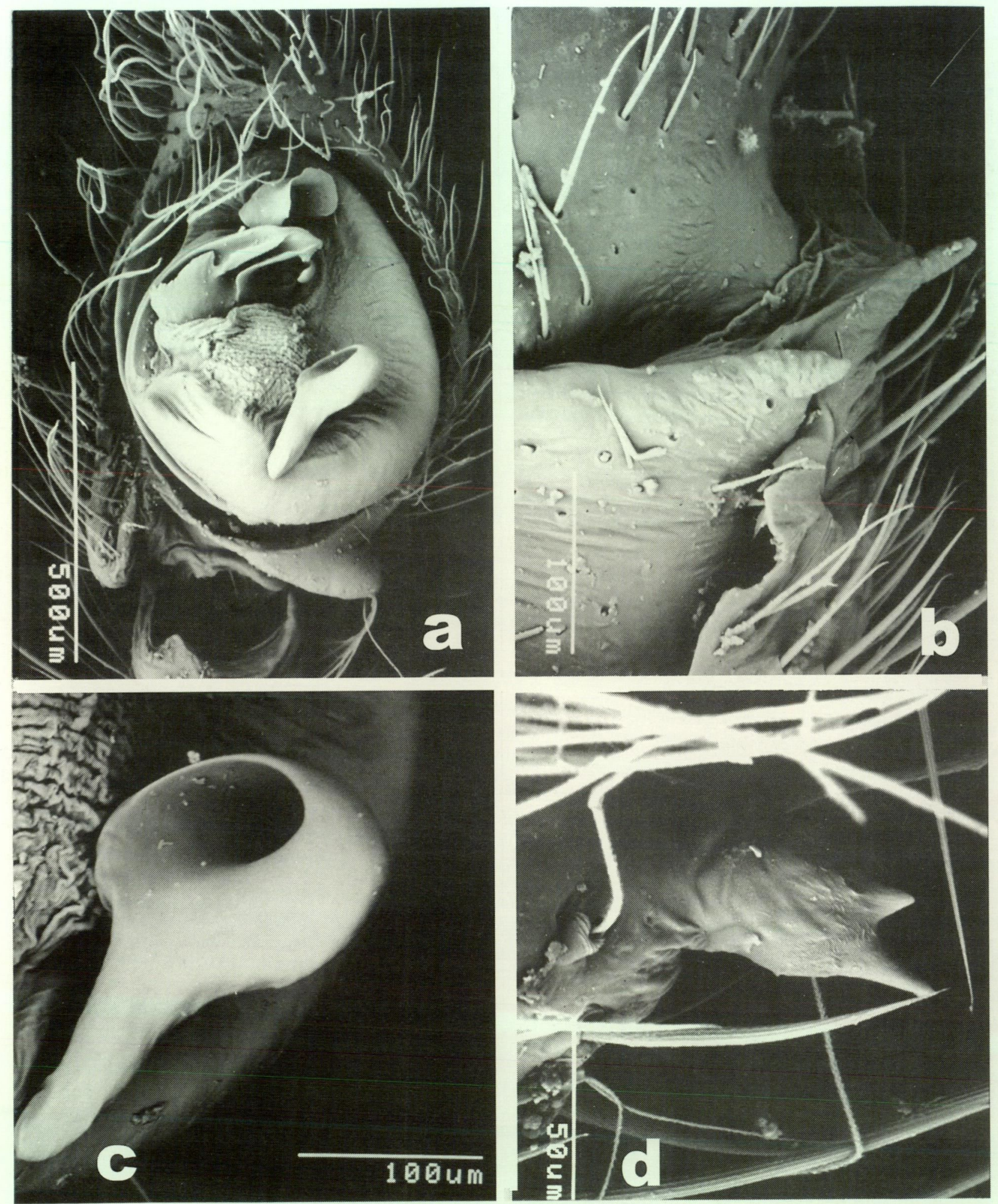

Figure 22 Amauropelma monteithi sp. nov.: male palp, ventral right, scanning electron micrographs: a, cymbium, bulb and distal tibia, ventral view; b, patellar apophyses, ventral view; $c$, median apophysis, ventral view; $d$, tibial apophysis, ventral view.

sparse. Tarsal rod with globular tip at basal onefifth on all leg tarsi. Trochanters deeply notched, as wide as long. Tarsi enlarge distally. Spines. As for genus except as noted. I: fe pv1p1d3r2w; ti p1r1v2.2.2.2; me p1r1. II: fe p1d3 r1; ti p1r1v2.2.2.2; me p1r1. III: fe p3d3r3; pa r1; ti p2d3r2; me p4r4v7. IV: fe p4d3r2; pa r1; ti p2d3 r2v2.2.2; me p4r4v9.
Palp: fe p1d2; pa 0; ti p2r1. Claws. Long, slender, gently and uniformly curved; small but distinct claw tufts set well below claws, few hairs around claws. Very prominent anvil-like onychium. Claws reflexed back as in Orsolobidae. 4-6 long close teeth on claws. Palp. Patella has ventral flange distally and also distally on retro-edge a small 
erect cone directed orthogonal to length. Tibia prolaterally flattened, incrassate on dorsal. Tibial apophysis on subdistal retrodorsal mound, apophysis small apically with shallow division into two cones, one very small basal. Cymbium tear-shaped, without dorsal scopula but with conical hirsute uniformly sclerotised apex and subapical spines; large rounded triangular basal process retroventrally-laterally directed; on proventral basal corner another smaller lobe directly back along length of tibia with spoon-like process distal which presses against dorsal of
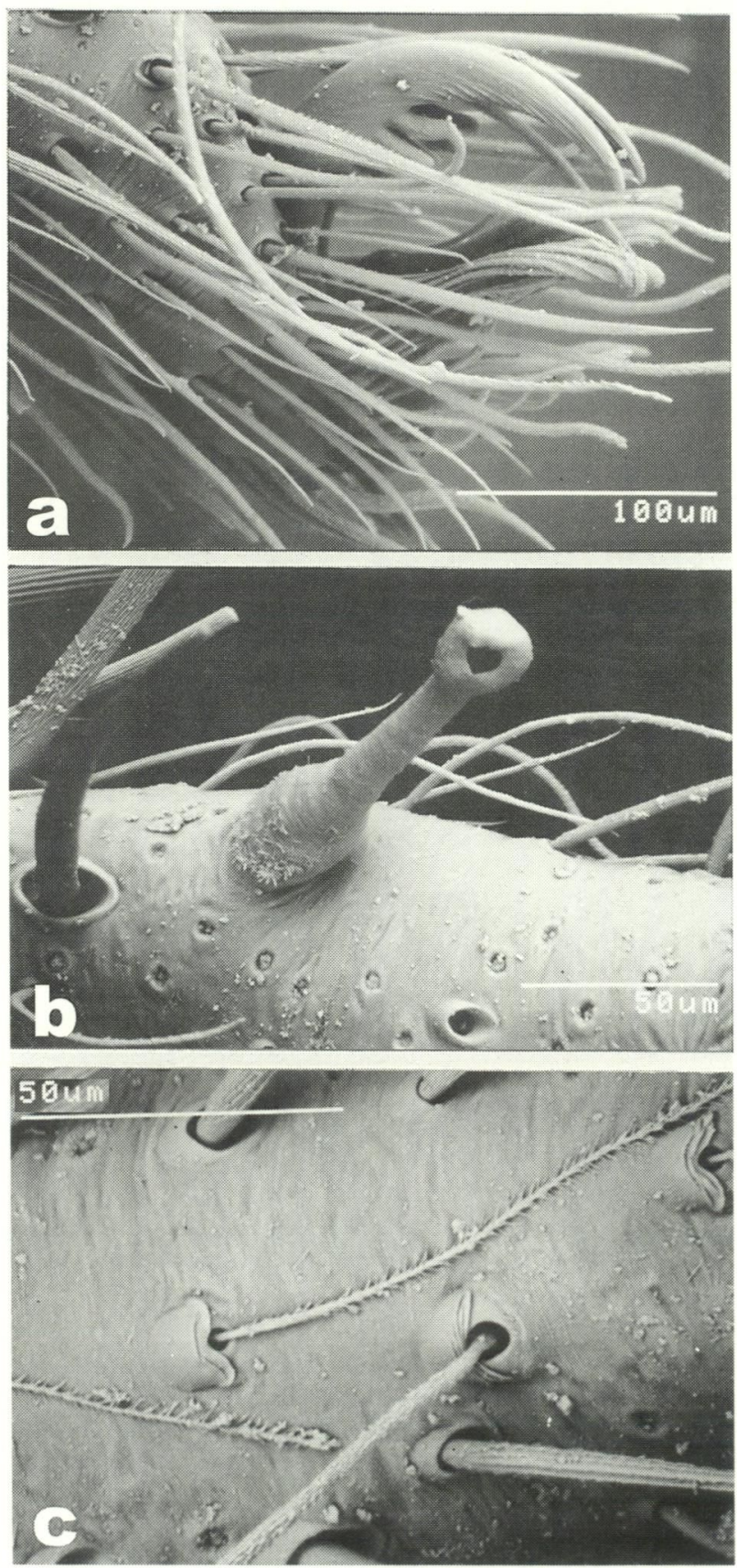

Figure 23 Amauropelma monteithi sp. nov. Female, scanning electron micrographs: a, distal tarsus, paired claws and claw tufts; $b$, tarsal rod; c, adpressed and erect trichobothria on tarsus. lateral cymbial lobe. Cymbial groove absent but retroventral lobe arises from flange continuing under bulb and dividing and curving to prolateral basal corner of cymbium ending free. Spinnerets. ALS c. equal to PLS in length but ALS thicker, PMS cylinders. All apices domed. ALS c.1 diameter apart noticeably. Trichobothria. 2 lines on tibia for length; one line for length of metatarsi; 2 rows, all with trichae of normal length, on tarsi.

\section{Allotype female (QM S24866)}

As for male except as follows:

Carapace 2.73 long, 1.88 wide. Abdomen 2.57, 1.73 wide.

Eyes. AME:ALE:PME:PLE, 5:4:3:5. Spines. As for genus except as noted. I: fe pv1p1d3. II: fe p1d3. III: fe pv1p1d3r2; pa p1r1; ti p2d3r2; me p4d1r3 v2.2.2. IV: fe p2d3r1; pa r1; ti p2d2r2; me p4r5v2.2.2. Palp: fe p1d3; pa 0; ti p2d1; ta p3. Epigyne. Externally broad plate or shield with wide wings and distomedial extension lateral of which are two small horns (copulatory channel) with ventral aperture. From copulatory fossae, duct passes forward to small marginal lobe and narrow duct passes back under folded edge of sclerotised shield to distal spherical receptaculum with fertilisation duct off basal inner margin.

\section{Distribution and Habitat}

Known only from rainforest at Cape Tribulation, north-eastern Queensland.

\section{Etymology}

In honour of Dr Geoff Monteith whose intensive and extensive collections greatly enhance studies on rainforests of north-eastern Australia.

\section{Amauropelma mossman}

Raven and Stumkat, sp. nov. Figures 2, 4, 24-26, Table 11

\section{Material Examined}

\section{Holotype}

ऽे, Mossman Bluff track, site 2, Queensland, Australia, $16^{\circ} 25^{\prime} \mathrm{S}, 145^{\circ} 19^{\prime} \mathrm{E}$, flight intercept trap, 20 December 1989-15 January 1990, G. Monteith, G. Thompson, ANZSES Expedition (QM S31361).

\section{Paratypes}

Australia: Queensland: $1 \delta$, Mossman Bluff track, Site $2,16^{\circ} 25^{\prime} \mathrm{S}, 145^{\circ} 19^{\prime} \mathrm{E}$, flight intercept trap, 20 December 1989-15 January 1990, G. Monteith, G. Thompson, ANZSES Expedition (QM S32916); 1 \%, Mossman Bluff track, Site 2, $16^{\circ} 25^{\prime} \mathrm{S}, 145^{\circ} 19^{\prime} \mathrm{E}$, flight intercept trap, 20 December 1989-15 January 1990, G. Monteith, G. Thompson, ANZSES Expedition (QMS31361); 1 б, Mossman Bluff Track, 5-10 km 

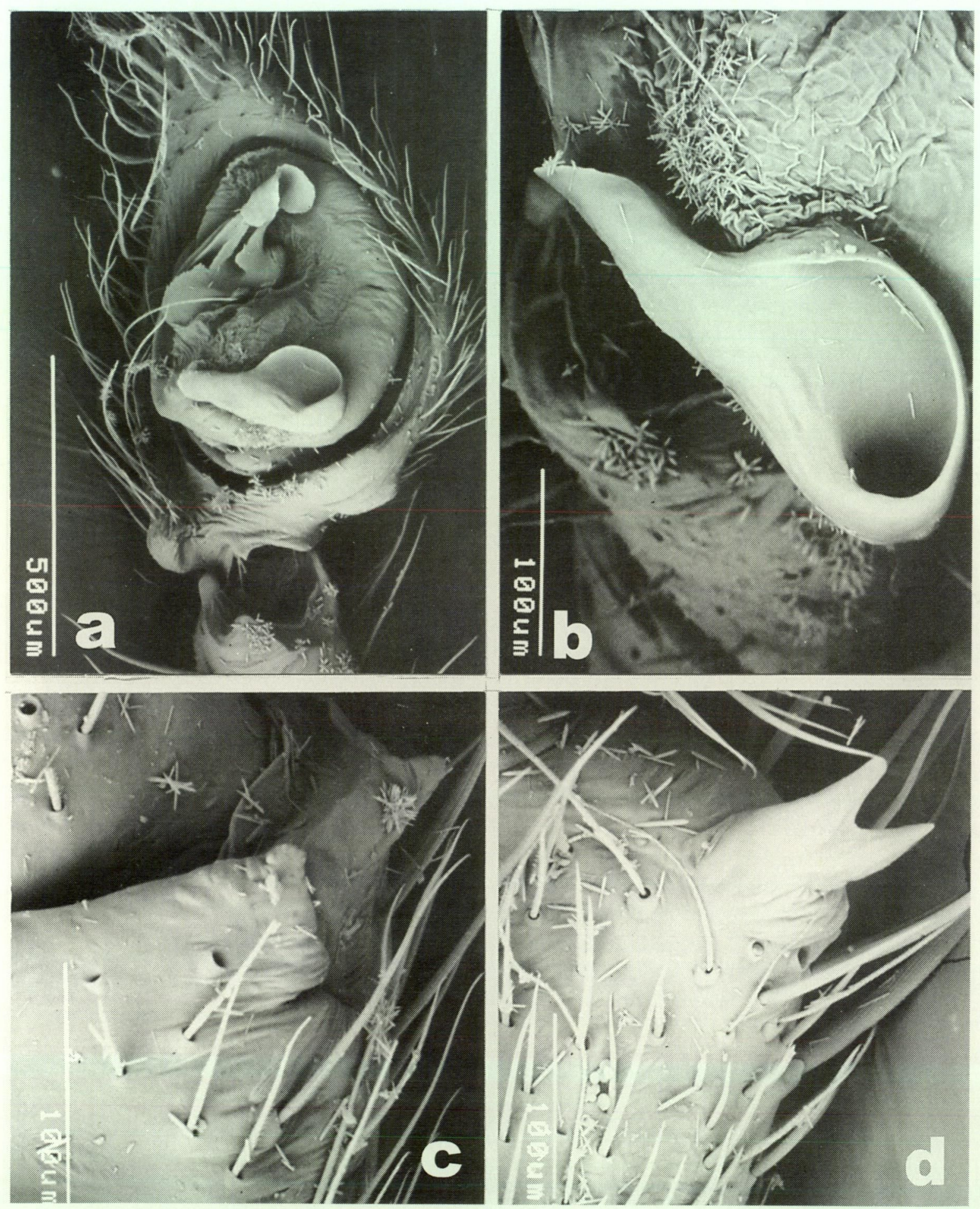

Figure 24 Amauropelma mossman sp. nov.: male palp, ventral right, scanning electron micrographs: a, cymbium, bulb and distal tibia, ventral view; b, median apophysis, rotated ventral view; $c$, patellar apophyses, ventral view; $d$, tibial apophysis, ventral view.

W. Mossman (Site 2), $16^{\circ} 25^{\prime} \mathrm{S}, 145^{\circ} 19^{\prime} \mathrm{E}$, flight intercept trap, 16-30 December 1988, G. Monteith, G. Thompson, ANZSES Expedition (QM S41787); 1 o. Clacherty $\mathrm{Rd}$, via Julatten, $16^{\circ} 37^{\prime} \mathrm{S}, 145^{\circ} 19^{\prime} \mathrm{E}$, rainforest, sieved litter, 11 October 1980, G. Monteith (QM S32895); 1 o, Mossman Bluff track (Site 3), $16^{\circ} 25^{\prime} \mathrm{S}, 145^{\circ} 22^{\prime} \mathrm{E}$, pitfall, $16-30$ December
1988, G. Monteith, G. Thompson, ANZSES Expedition (QM S32894); 1 ㅇ, Mossman Bluff track (site 3), $16^{\circ} 25^{\prime} \mathrm{S}, 145^{\circ} 22^{\prime} \mathrm{E}$, pitfall, 16-30 December 1988, G. Monteith, G. Thompson, ANZSES Expedition (QM S32893); 2 \&, Mossman Bluff track (Site 3), $16^{\circ} 25^{\prime} \mathrm{S}, 145^{\circ} 22^{\prime} \mathrm{E}$, pitfall, 16-30 December 1988, G. Monteith, G. Thompson, ANZSES 
Expedition (QM S32890); 1 ㅇ, Mossman Gorge NP (NQ 15), $16^{\circ} 28^{\prime} \mathrm{S}, 145^{\circ} 19^{\prime} \mathrm{E}, 1$ November 1991-22 July 1992, P. Lawless, R. Raven, M. Shaw (QM S32892); 1 \& . Mossman Gorge NP (NQ 15), 16 28'S, $145^{\circ} 19^{\prime} \mathrm{E}, 1$ November 1991-22 July 1992, P. Lawless, R. Raven, M. Shaw (QM 532891.

\section{Diagnosis}

Males differ from those of $A$. trueloves in the size of the retrobasal cymbial process and that the median apophysis is horizontal rather than vertical and from those of $A$. hasenpuschi in the dominant 'handle' on the median apophysis. Females differ from those of $A$. wallaman by the PME clearly being larger than the other eyes.

\section{Description}

Holotype male (QM S31361)

Carapace 2.38 long, 1.85 wide. Abdomen 2.00, 1.38 wide.

Colour. Carapace yellowish with wide irregular brown margin and wide brown lateral bands from anterior margins of carapace onto caput and then bracketing fovea; chelicerae yellow brown with dark medial band down each. Abdomen dorsally mottled brown and white with large areas of white mottling anteriorly, ventrally pallid with small dark mottling laterally and posteriorly. Legs yellow brown; femora with brown areas ventrally in undulating waves laterally, two darker bands on tibiae. Carapace. Margins hirsute. Eyes. AME set close on common tubercle. From above, ALE set
Table 11 Leg measurements of Amauropelma mossman.

Holotype male (QM S31361).

\begin{tabular}{llllll}
\hline & Leg 1 & Leg 2 & Leg 3 & Leg 4 & Palp \\
\hline Femur & 1.92 & 1.60 & 1.52 & 2.12 & 0.84 \\
Patella & 0.92 & 0.84 & 0.72 & 0.84 & 0.40 \\
Tibia & 1.72 & 1.32 & 1.20 & 1.72 & 0.28 \\
Metatarsus & 1.36 & 1.24 & 1.32 & 2.00 & \\
Tarsus & 0.72 & 0.68 & 1.20 & 1.20 & 0.84 \\
\hline Total & 6.64 & 5.68 & 5.52 & 7.88 & 2.36 \\
\hline
\end{tabular}

Allotype female (QM S32893).

\begin{tabular}{lccccc}
\hline & Leg 1 & Leg 2 & Leg 3 & Leg 4 & Palp \\
\hline Femur & 1.68 & 1.44 & 1.24 & 1.76 & 0.60 \\
Patella & 0.80 & 0.80 & 0.72 & 0.84 & 0.40 \\
Tibia & 1.36 & 1.08 & 1.00 & 1.64 & 0.40 \\
Metatarsus & 0.96 & 0.96 & 1.04 & 2.00 & \\
Tarsus & 0.48 & 0.40 & 0.92 & 0.92 & 0.56 \\
\hline Total & 5.28 & 4.68 & 4.72 & 7.16 & 1.96 \\
\hline
\end{tabular}

behind anterior edge of PME, tuberle of ALE and PLE touch. AME:ALE:PME:PLE, 7:6:8:6. Spines. As for genus except as follows. I: fe pv1p1d3r1. II: fe pv1p1d3r1. III: fe p1p2d3r3. IV: fe pv1p2d3r2. Palp. Patella with one distinct cone, ventral corner of fold not enlarged. Tibia with retrolateral apophysis with wide base, apically bifid with short similar apices. Cymbium retrobasally with rounded rectangular lobe, probasal lobe small, rounded; two spines

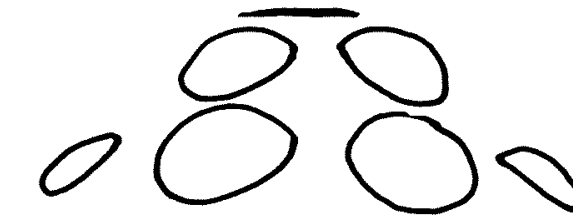

O

a

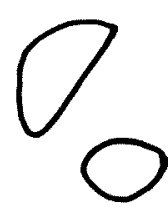

b
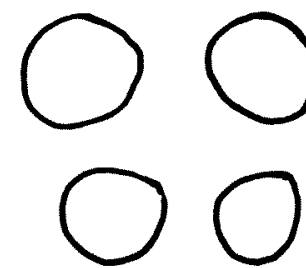
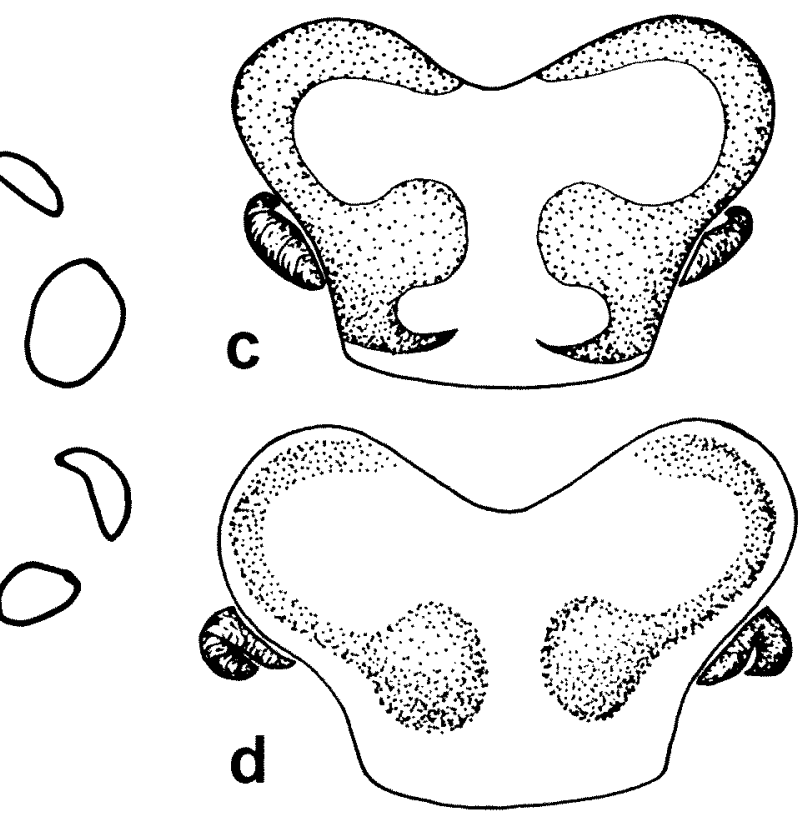

Figure 25 Amauropelma mossman sp. nov., a, b, holotype male: eyes, dorsal view (a), frontal view (b); c, d, allotype female, epigyne, dorsal view (c), ventral view (d). Scale line $=0.3 \mathrm{~mm}$. 

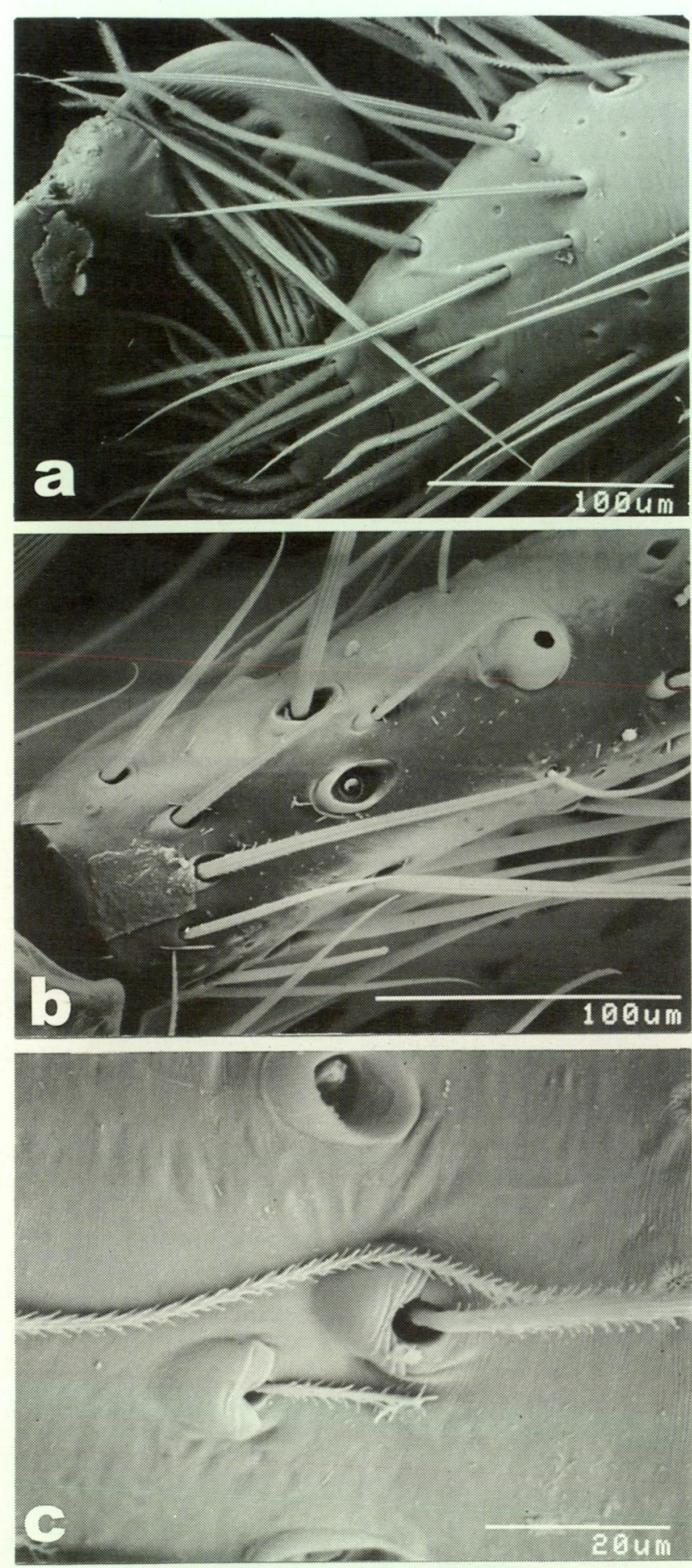

Figure 26 Amauropelma mossman sp. nov. Female, scanning electron micrographs: a, distal tarsus, paired claws and claw tufts; $b$, tarsal organ; c, adpressed and erect trichobothria.

apico-ventrally on cone. Embolic plate short, beaklike with small foliform conductor; median apophysis set on ventral edge of low C-shaped tegulum, long distinct but tapering handle, lumen subcircular.

\section{Allotype female (QM S32893)}

Carapace 2.40 long, 1.76 wide. Abdomen 3.00 long, 2.00 wide. Total length 5.2.
Colour. Carapace yellow with dark narrow marginal and subcentral bands. Abdomen dorsally light green brown with large white mottling. Femora yellow with pale dark finger spots basally, and incomplete band subdistally and distally. Carapace. Fovea high, with saddle anteriorly. Eyes. 2.4.2. $\mathrm{PME}=\mathrm{AME}>\mathrm{AME}=\mathrm{PLE}=\mathrm{ALE}$. ALE-PLE $<$ ALE-AME. From above ALE set behind PME. Back eyes in two well separated rows. ALE look down and to side. From front, lower edge of ALE above upper edge of AME. Eye group $=0.7$ of head width; PME $=12 \%$ of head width. Legs. Tarsi narrow for basal $1 / 6$, widening just distal of tarsal organ at $1 / 5$. Low but large, distinct tarsal organ in basal $1 / 3$ on I; more posteriorly on legs II-IV. All trochanters deeply notched. Spines. As for genus except as noted. Tibia I, II with large paired spines overlapping and on raised bases, 4 long predistally and 1 shorter distal; metatarsi with same but only 3 pairs. Pair of strong erect spines proventrally on femur I. I: fe p1pv2d3. II: fe p1d3. III: fe pv1p2d3r1; pa r1; ti p2d2r2v1.2.2; me p3d1r3v2.2.2. IV: fe p2d3r1; pa r1; ti p2d2r2v1.2.2; me p3d1r3v2.2.2. Palp: fe d1.2; pa p1; ti p2d2; ta p1.2. Epigyne. Plate about 1.5 times wider than long; anterior edge deeply U-shaped; basolateral edge concave with narrow distal edge; large rounded lateral wings; lateral horns fleshy, set anteriorly and wide of wings. Spinnerets. ALS distinctly separated.

\section{Distribution and Habitat}

Known only from montane rainforest west of Mossman, north-eastern Queensland.

\section{Etymology}

A noun in apposition taken from the type locality.

Amauropelma pineck Raven and Stumkat, sp. nov. Figures 4, 27a, b, d, 28d, Table 12

\section{Material Examined}

\section{Holotype}

๙ิ, Pine Ck, CSIRO Tower, Queensland, Australia, $17^{\circ} 00^{\prime} \mathrm{S}, 145^{\circ} 50^{\prime} \mathrm{E}$, pitfall and intercept traps, 12 September 1991-20 October 1991, G. Monteith, H. Janetzki (QMS 32906).

\section{Paratypes}

Australia: Queensland: 4 , same data (QM S39333); 1 \&, Crystal Cascades, $16^{\circ} 57^{\prime} \mathrm{S}, 145^{\circ} 40^{\prime} \mathrm{E}$, pitfall, 27 October 1991-23 July 1992, R. Raven, P. Lawless, M. Shaw (QM S24529); 1 \&, same data except 23 July 1992-26 November 1992, R. Raven, P. Lawless, E. Lawless, M. Shaw (QM S24016); 3 ㅇ, same data except 26 November 1992-16 April 1993, R., J., and S. Raven, P. and E. Lawless (QM S24882, 24880, WAM T42602). Mission Beach (S1), 1752'S, 
$146^{\circ} 03^{\prime} \mathrm{E}$, rainforest, pitfall, 29 July-2 September 1996, M. Cermak: 2 ơ (QM S39103); 1 \& (QM S41791); 1 ? (QM S41792); 1 oे (QM S39102 now WAM T41616); 3 oे (QM S41790); 1 ?, same but 119 July 1996 (QM S41789).

\section{Diagnosis}

Differs from A. trueloves having only four pairs of spines ventrally on tibiae I, II.

\section{Description}

\section{Holotype male (QM S32906)}

Carapace 2.52 long, 1.92 wide. Abdomen 1.84 long, 1.32 wide. Total length 4.6 .

Colour. Carapace yellow brown with narrow dark marginal bands and wide dark bands subcentrally. Abdomen dorsally light greenish brown with large paired four irregular pallid marks. Legs yellow brown with dark shadows medially and distally on femora, laterally on patellae and basally and distally on tibiae, especially on III, IV. Eyes. 2.4.2. AME set
Table 12 Leg measurements of Amauropelma pineck,

Holotype male (QM S32906).

\begin{tabular}{llllll}
\hline & Leg 1 & Leg 2 & Leg 3 & Leg 4 & Palp \\
\hline Femur & 1.80 & 1.76 & 1.72 & 2.24 & 0.80 \\
Patella & 0.92 & 0.84 & 0.80 & 0.96 & 0.40 \\
Tibia & 1.88 & 1.40 & 1.16 & 1.88 & 0.32 \\
Metatarsus & 1.44 & 1.28 & 1.36 & 2.28 & \\
Tarsus & 0.72 & 0.64 & 1.04 & 1.04 & 0.80 \\
\hline Total & 6.76 & 5.92 & 5.80 & 8.40 & 2.32 \\
\hline
\end{tabular}

Allotype female (QM S39333).

\begin{tabular}{lccccc}
\hline & Leg 1 & Leg 2 & Leg 3 & Leg 4 & Palp \\
\hline Femur & 2.00 & 1.80 & 1.68 & 2.40 & 0.84 \\
Patella & 1.04 & 0.96 & 0.80 & 1.24 & 0.60 \\
Tibia & 1.76 & 1.40 & 1.20 & 1.90 & 0.44 \\
Metatarsus & 1.24 & 1.20 & 1.40 & 2.16 & \\
Tarsus & 0.56 & 0.56 & 1.16 & 1.16 & 0.68 \\
\hline Total & 6.60 & 5.92 & 5.84 & 8.86 & 2.56
\end{tabular}
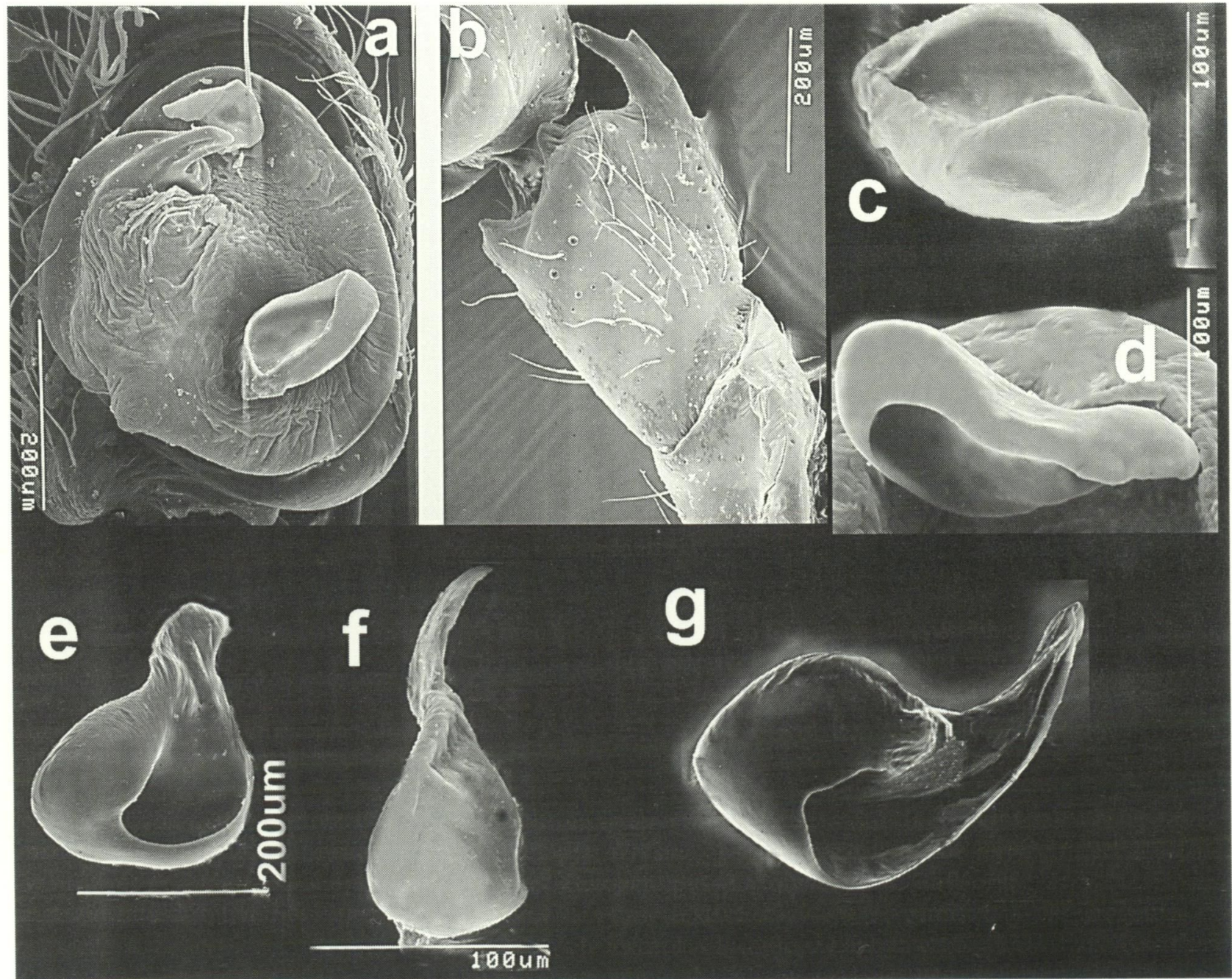

Figure 27 Amauropelma spp. nov.: scanning electron micrographs. A. pineck sp. nov., a, b, d. Male palp, ventral right: a, cymbium and bulb, ventral view; $b$, distal patella and tibia, retrolateral view; $c-g$, median apophysis with lumen; c, A. mcilwraith; d, A. pineck; e, A. torbjorni; f, A. gayundah, lumen absent; g, A. rifleck; f, g, same scale. 
forward on common tubercle. MOQ subquadrate. ALE clearly closer to PLE than AME. PME $>$ PLE=AME $>$ ALE. From front, lower edge of ALE cut above centres of AME. Back row just forming two rows. Chelicerae. 4 teeth on retromargin. Palp. Patella without noticeable apophysis. Tibia with retrodorsal conical process with small thorn dorsally at half length. Cymbium ascopulate; prolateral basal lobe squared, retrobasal process rounded, noticeably produced; two spines below cymbial tip. Lumen of subquadrate median apophysis dominant. Legs. Tarsal organ at distal third (I), distal 2/5 (II), or basal 1/8 (III, IV). Scopula absent. Spines. As for genus except, I: fe pv1d1d3; II, fe pv1p1d2; ti p1. III: fe p2d3r1; pa r1; ti p2d3r2v2.2.2; me p1.1.2r1.1.2.v2.2.2. IV as III but: fe p2d3r2; me p1.1.2r1.2.2.v2.1.1.1.2. Palp: fe d1.1; pa p1; ti p1; cymbium v2.

\section{Allotype female (QM S39333)}

Like male except as follows:

Carapace 2.60 long, 2.08 wide. Abdomen 2.60 long, 2.00 wide. Total length 5.9. Eyes. Eye group/ head width $=0.63$; PME $=9 \%$ of head width. Legs. Patterns darker, distal spines ventrally on tibiae I, II absent. Spines. I: fe pv1p1d2; ti v2.2.2.2. II: fe p1d2; ti v2.2.2.2. III: pv1p2d3r1. IV: p1d3r1. Palp: fe d1.2; pa p1; ti p2d1; ta p2.1r.1. Epigyne. Two wide lobes or 'wings' with small lateral horns.

\section{Distribution and Habitat}

Known only from lowland rainforest near Cairns, north-eastern Queensland.

\section{Etymology}

A noun in apposition taken from the type locality.
Amauropelma rifleck Raven and Stumkat, sp. nov. Figures $3 c, d, 4,27 g, 28 a-c, 29$, Table 13

\section{Material Examined}

\section{Holotype}

$\delta$, Ellis Beach Rd (NQ 14/2), Queensland, Australia, $16^{\circ} 40^{\prime} \mathrm{S}, 145^{\circ} 34^{\prime} \mathrm{E}$, pitfall, $22 \mathrm{July}-27$ November 1992, R. Raven, P. and E. Lawless, M. Shaw (QMS52203).

\section{Paratypes}

Australia: Queensland: 5 , same data (QM S21767, WAM T42603).

\section{Diagnosis}

Males differ from those of $A$. trueloves in the large bifid patellar process and median apophysis directed outward, females in the much wider epigynal plates and the ALE are clearly in front of the PME, not beside them as in A. torbjorni.

\section{Description}

\section{Holotype male (QMS21796)}

Carapace 2.68 long, 2.04 wide. Abdomen 1.80 long, 1.32 wide.

Colour. Carapace yellow brown with sheen with darker, wide subcentral band from eyes to posterior slope. Abdomen collapsed away from cuticle. Carapace. Very lightly hirsute. Eyes. AME on common tubercle. AME:ALE:PME:PLE, 6:5:5:5. From above, ALE set in front of PME; AME on separate mound to PLE. Legs. Without tarsal rod. Spines. As for genus except as noted. I: fe

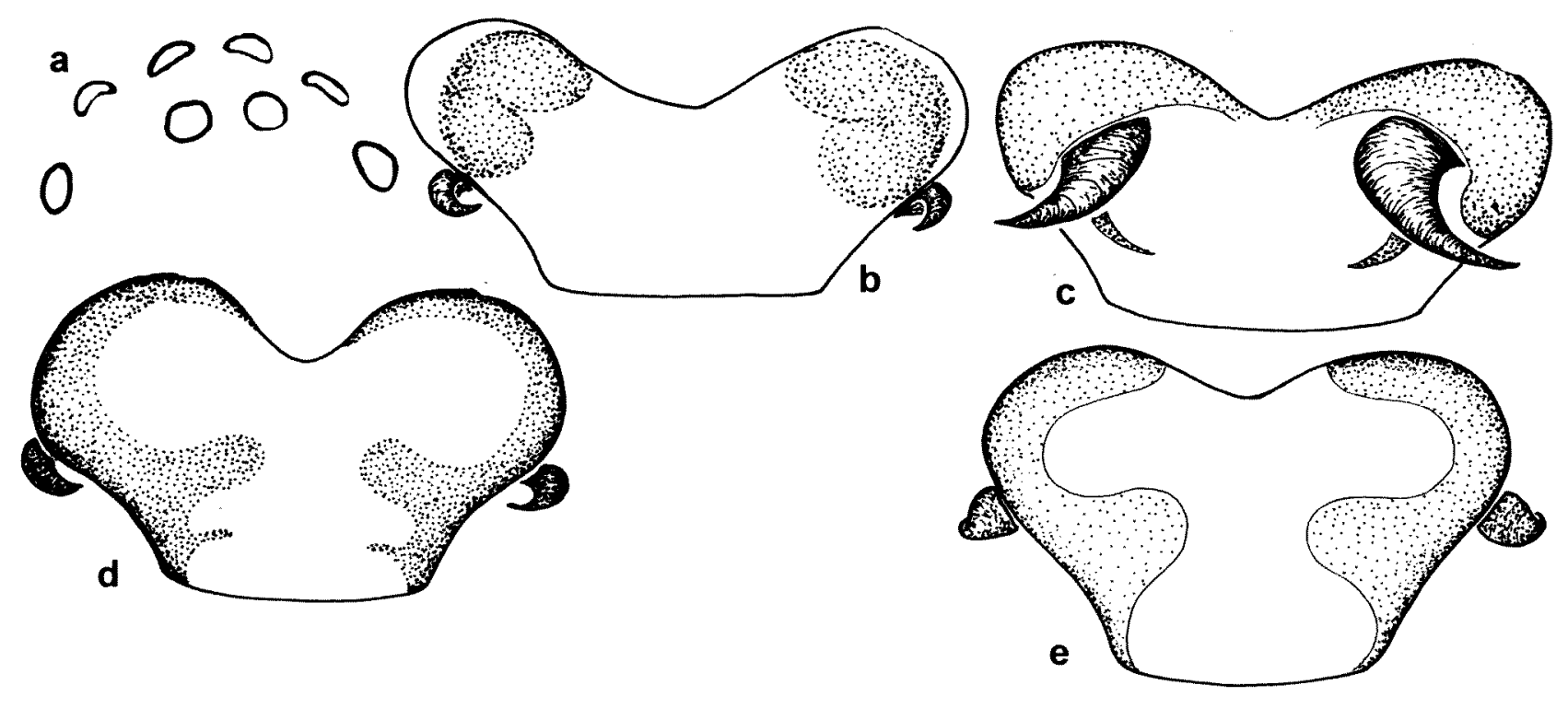

Figure 28 Amauropelma spp. nov. a-c, A. rifleck sp. nov.: holotype male (a), paratype female (QMS 21917 (b, c): a, eyes, dorsal view; b, c, epigyne, ventral view. (b), dorsal view (c); d, A. pineck sp. nov., female, epigyne, ventral view; e, A. wallaman sp. nov., female, epigyne, ventral view. Scale lines $=0.5 \mathrm{~mm}$ for $\mathrm{a}-\mathrm{c}, 0.3 \mathrm{~mm}$ for $\mathrm{d}$, e. 

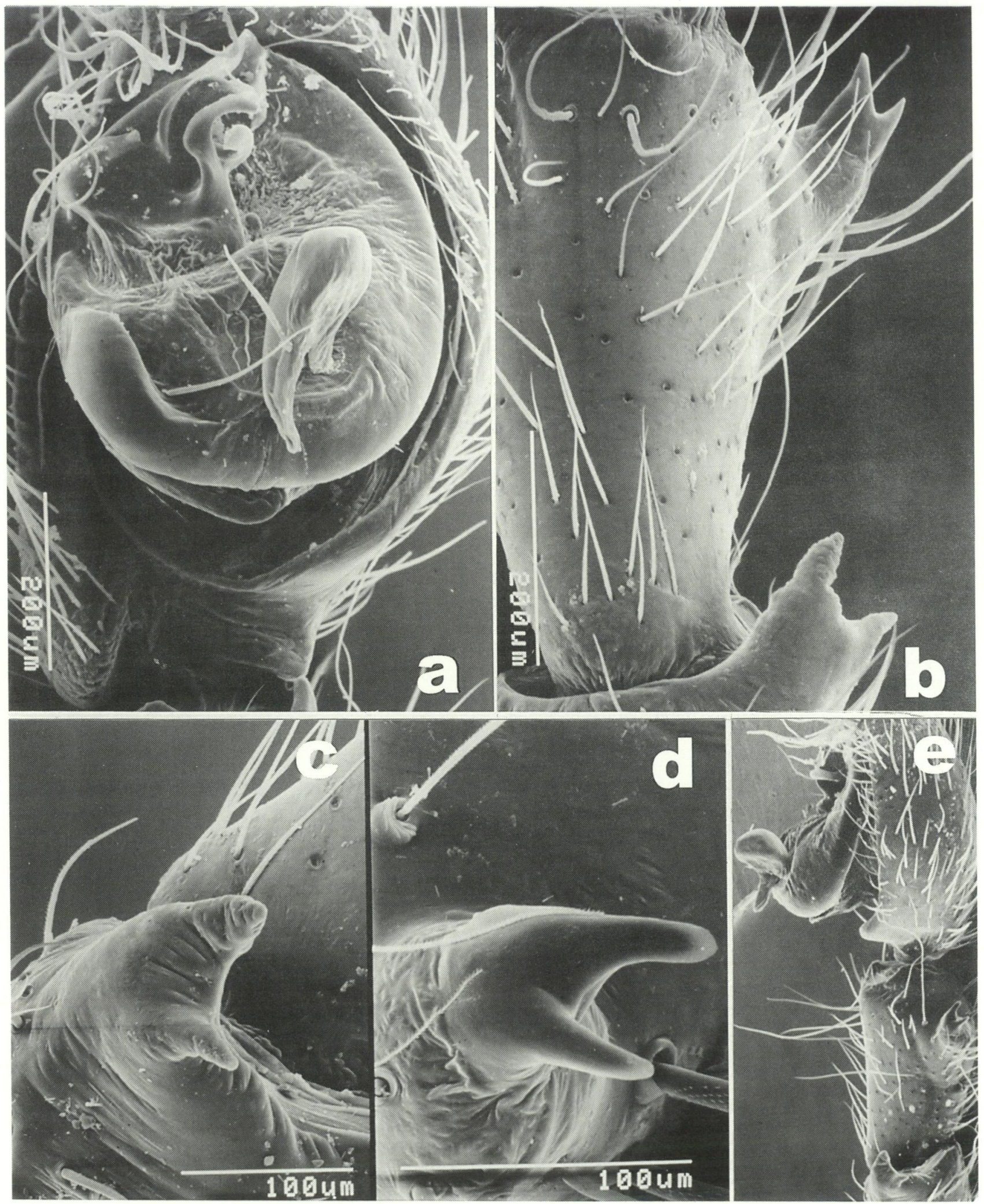

Figure 29 Amauropelma rifleck sp. nov.: male palp, ventral right, scanning electron micrographs: a, e, cymbium, bulb and distal tibia, ventral view (a), retrolateral view (e); b, tibia and patella showing apophyses, ventral view; $c$, patellar apophysis, retroventral view; d, tibial apophysis, retroventral view.

pv1p1d3r1; ti p1r1; me p1r1. II: fe pv1p1d3r1; ti p1r1; me p1r1. III: fe pv1p1d3r2; pa r1; ti p2d3r2v.2.2; me p2r2v.2.2. + distal whorl of 4. IV: fe pv1p2d3r2; pa 0; ti p2d3r2; me p2r2vv.2.2. + distal whorl of 4. Palp: fe p1d1.2; pa p1r1; cymbium ventroapical 2. Palp. Patella with single apically divided retrodistal process apically divided into two cones; tibial apophysis similar but slightly 
Table 13 Leg measurements of Amauropelma rifleck.

Holotype male.

\begin{tabular}{lccccc}
\hline & Leg 1 & Leg 2 & Leg 3 & Leg 4 & Palp \\
\hline Femur & 1.92 & 1.80 & 1.72 & 2.32 & 1.12 \\
Patella & 1.04 & 0.96 & 0.84 & 1.00 & 0.32 \\
Tibia & 1.88 & 1.52 & 1.20 & 2.04 & 0.40 \\
Metatarsus & 1.60 & 1.40 & 1.44 & 1.80 & \\
Tarsus & 0.80 & 0.72 & 0.80 & 1.00 & 0.96 \\
\hline Total & 7.24 & 6.40 & 6.80 & 8.16 & 2.80 \\
\hline
\end{tabular}

Allotype female (QM S21917).

\begin{tabular}{llllll}
\hline & Leg 1 & Leg 2 & Leg 3 & Leg 4 & Palp \\
\hline Femur & 1.84 & 1.72 & 1.48 & 2.24 & 0.88 \\
Patella & 1.00 & 0.92 & 0.80 & 0.92 & 0.44 \\
Tibia & 1.60 & 1.28 & 1.08 & 1.88 & 0.52 \\
Metatarsus & 1.20 & 1.12 & 1.00 & 1.92 & \\
Tarsus & 0.60 & 0.56 & 0.92 & 0.92 & 0.72 \\
\hline Total & 6.24 & 5.60 & 4.96 & 7.88 & 2.56 \\
\hline
\end{tabular}

longer. Cymbium without any retrobasal enlargement or lobe. Median apophysis longitudinal, pyriform with lumen not evident in ventral view.

\section{Allotype female (QM S21917)}

Carapace 2.44 long, 1.84 wide. Abdomen 2.80 long, 1.48 wide. Total length 5.5 .

Eyes. Eye group $=0.66$ of head width; $\mathrm{PME}=8 \%$ of head width. Spines. As for male but lacking lateral spines on tibiae and metatarsi I, II Legs. Tarsal organ at distal $1 / 3$ on I, distal $3 / 8$ on II, basal on III, IV. Epigyne. Plate with wide wings.

\section{Distribution and Habitat}

Known only from dense grassy open forest at Ellis Beach, north of Cairns, north-eastern Queensland.

\section{Etymology}

A noun in apposition taken from the type locality.

\section{Amauropelma torbjorni Raven and Gray, sp. nov.} Figures 4, 27e, 30, 31, Table 14

\section{Material Examined}

\section{Holotype}

ऽ, Keatings Gap, Mt Cook, Queensland, Australia, $15^{\circ} 30^{\prime} \mathrm{S}, 145^{\circ} 13^{\prime} \mathrm{E}$, pitfall, 20 July 1992-28 November 1992, R. Raven, P. Lawless, E. Lawless, M. Shaw (QMS24364).

\section{Allotype}

Australia: Queensland: $q$, Quarantine Rd, Mt
Cook, $15^{\circ} 30^{\prime} \mathrm{S}, 145^{\circ} 13^{\prime} \mathrm{E}$, pitfall, 28 November 199218 April 1993, R., J. and S. Raven, P. and E. Lawless (QM S24062).

\section{Diagnosis}

Males differs from those of $A$. trueloves in larger patellar apophysis; females have a relatively shorter epigyne than those of $A$. rifleck and the ALE are beside PME not in front as in $A$. rifleck.

\section{Description}

\section{Holotype male (QM S24364)}

Carapace 3.24 long, 2.68 wide.

Colour. Carapace and chelicerae yellow orange with dark lines on interstrial edges. Abdomen collapsed but pallid with dark areas dorsally and mostly pallid ventrally. Legs yellow orange without banding or pattern. Eyes. Small; AME set close on common tubercle; from above and in front ALE are beside PME; from in front ALE are transverse and ovoid. AME:ALE:PME:PLE, 6:5:6:4. Spines. As for genus except as noted. Very long, strong, on metatarsi especially on III. I: fe p1d2r1; ti p1r1; me p1r1. II: fe p1d4; pa 0; ti p1r1; me p3r2. III: fe pv1p2d4r1; pa r1; ti p2d3r2; me p1.1.2r1.2v2.2.2.2, short pair medially. IV: missing. Palp: fe p1d3, pa p1w, ti p2w, cymbium 0 distoventral. Palp. Patella with single strong coniform retrodistal process and well separated small ventral prong. Retrolateral tibial apophysis strong, bifurcate with two similar tips; tibia excavate retrolaterally. Strong curved coniform retrobasal process on cymbium and broad rounded probasal process; embolic plate a folded cone with broad translucent conductor, then median apophysis transverse with extensive cavity but overall anvil-shaped with short handle.

\section{Allotype female (QMS 24062)}

Like male but:

Spines. As for genus except as noted. I: fe pv1p1d3w. II: fe p1d3w; me v2.2.4. III: fe pv1p2d3r1; pa r1; ti p2d2r2; me p1.1.2d1r1. 1.2v2.2.4. IV: fe p1d3r1; pa r1; ti p2d3r2v2.2; me p1.1.2d1r1.1.2v1.1.1.2.2.2. Palp: fe p1d3; pa0; ti p2;

Table 14 Leg measurements of Amauropelma torbjorni.

\begin{tabular}{lcclll}
\hline \multicolumn{7}{l}{ Holotype male (QM S24364). } \\
\hline & Leg 1 & Leg 2 & Leg 3 & Leg 4 & Palp \\
\hline Femur & 2.72 & 2.44 & 2.32 & - & 1.24 \\
Patella & 1.40 & 1.28 & 1.08 & - & 0.76 \\
Tibia & 2.60 & 2.00 & 1.68 & - & 0.56 \\
Metatarsus & 2.04 & 1.80 & 1.92 & - & \\
Tarsus & 1.00 & 0.84 & 0.00 & - & 1.24 \\
\hline Total & 9.76 & 8.36 & 7.80 & - & 3.80 \\
\hline
\end{tabular}




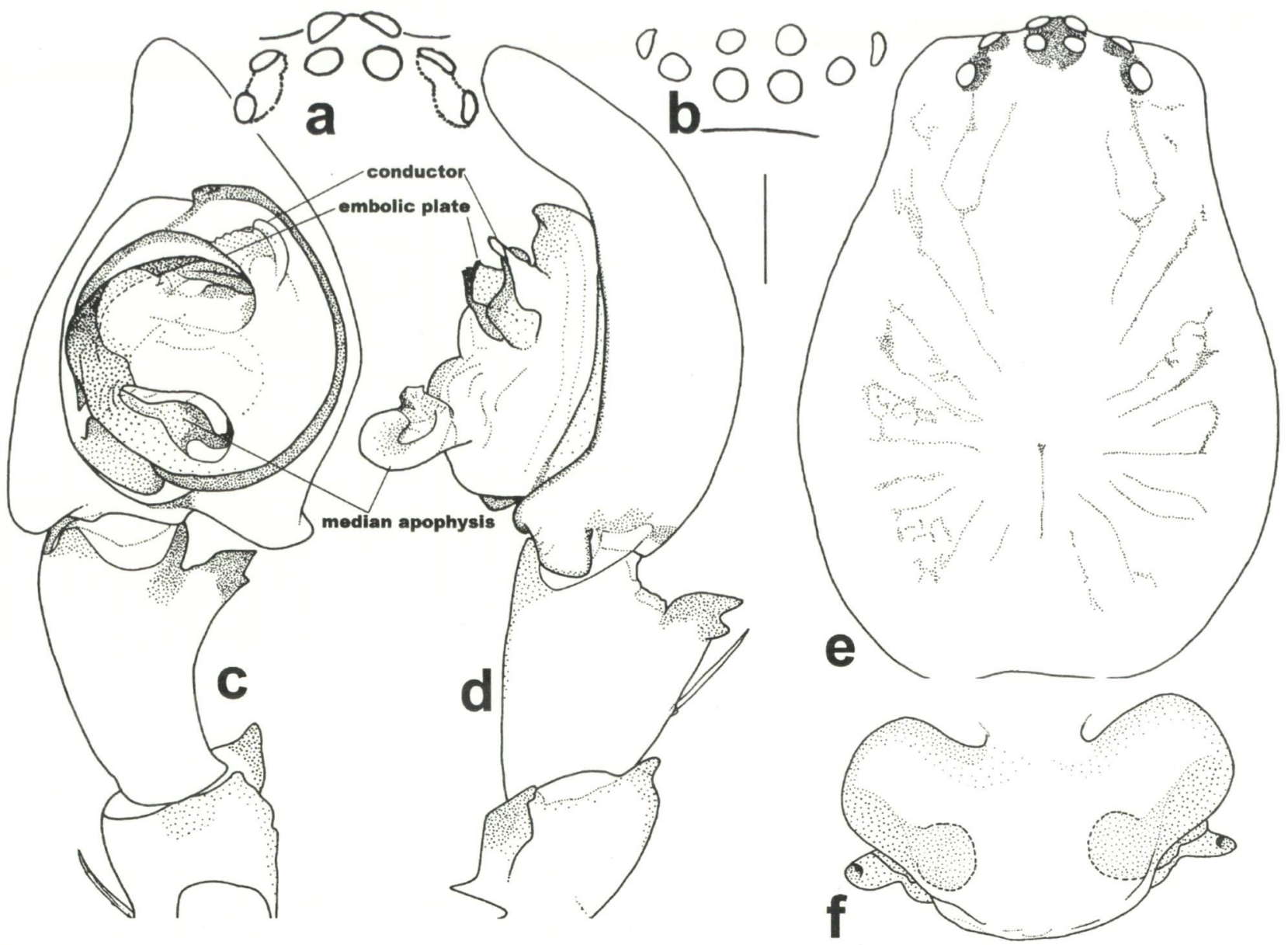

Figure 30 Amauropelma torbjorni sp. nov. a, eyes, dorsal view; b, eyes, frontal view; c, d, male palpal patella to cymbium, ventral view (c), retrolateral view (d); e, male carapace, dorsal view; f, epigyne, ventral view. Scales $0.32 \mathrm{~mm}$ for $\mathrm{a}, \mathrm{b}, 0.25$ for rest.
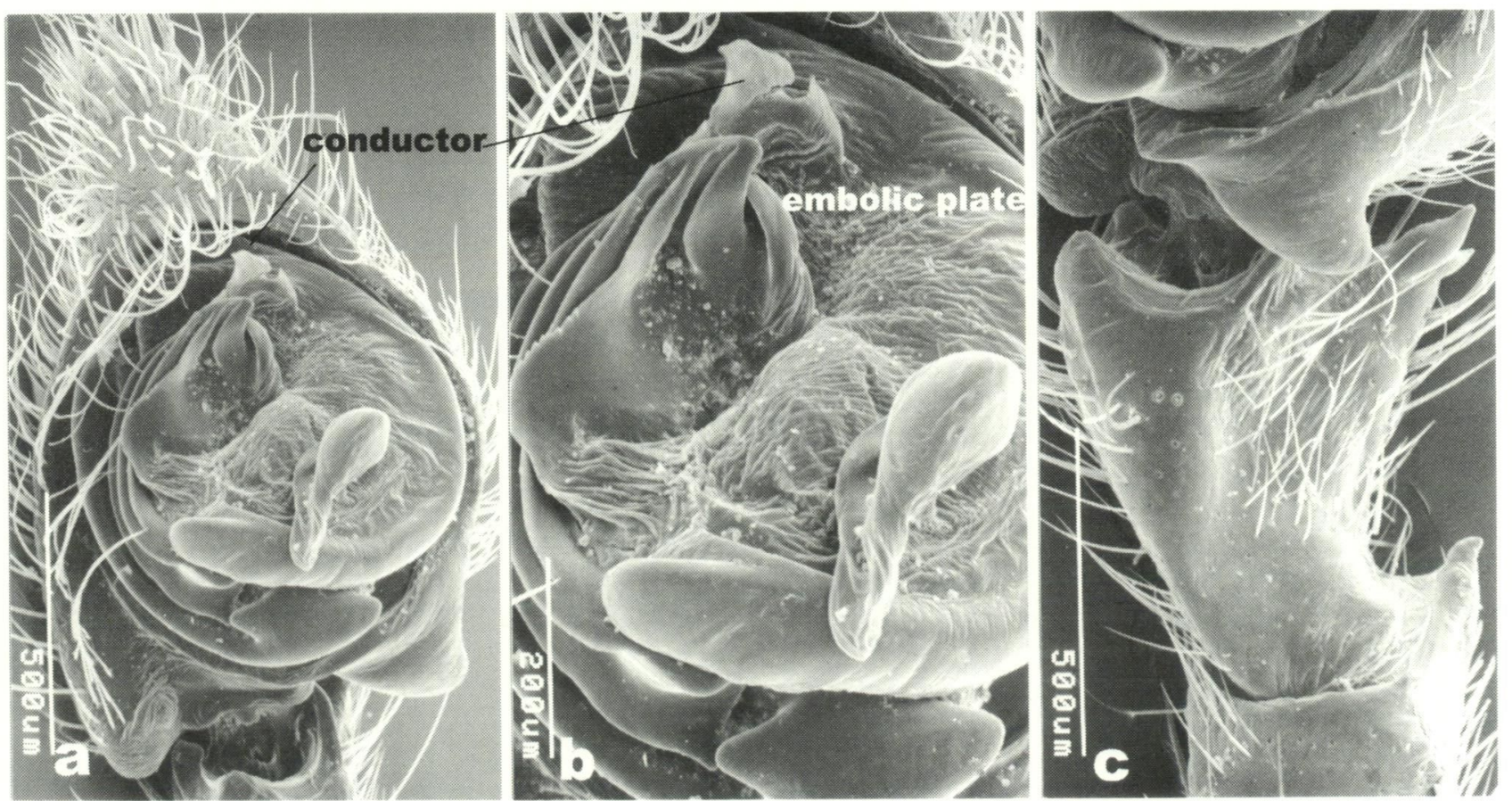

Figure 31 Amauropelma torbjorni sp. nov.: male palp, ventral right, scanning electron micrographs: a, cymbium, bulb and distal tibia, ventral view; b, embolic plate, conductor and median apophysis, ventral view; $c$, palpal tibia and patella showing apophyses, ventral view. 
ta v2. Epigyne. A broadly rounded plate, wider than long and anteriorly concave, with wide lateral wings and small lateral horns set close to plate.

\section{Distribution and Habitat}

Known only from rainforest (type) at Mt Cook, near Cooktown, north-eastern Queensland.

\section{Etymology}

Named for Dr Torbjörn Kronestedt, who came to mind while RJR was at the type locality.

\section{Amauropelma undara Raven and Gray, sp. nov.} Figures 4, 32, 33a-c, e-g, Table 15

\section{Material Examined}

\section{Holotype}

$\delta$, Undara Lava Tubes, Bayliss Cave, Queensland,
Australia, $18^{\circ} 25^{\prime} \mathrm{S}, 144^{\circ} 28^{\prime} \mathrm{E}, 8$ February 1996 , E. Volschenk, D. Slaney (QM S31389).

\section{Paratypes}

Australia: Queensland: same locality as for holotype: 1 \&, 27 May 1986, F.G. Howarth, D. Irvin (QM S46226); 1 \%, 1 \&, 12-13 June 1986, F.G. Howarth, D. Irvin, S. Robson (AM KS50696); 2 , 15 June 1985, F.G. Howarth, D. Irvin, J. Bresnan (AM KS50693, AM KS50694); 1 \&, 31 May 1986, F.G. Howarth, D. Irvin (AM KS56317); 1 ঠ, 14 June 1985, F.G. Howarth, D. Irvin, J. Bresnan (AM KS50692).

\section{Other Material}

Australia: Queensland: same locality as for holotype: 1 juvenile, 5 June 1986, F.G. Howarth (QM S46227).

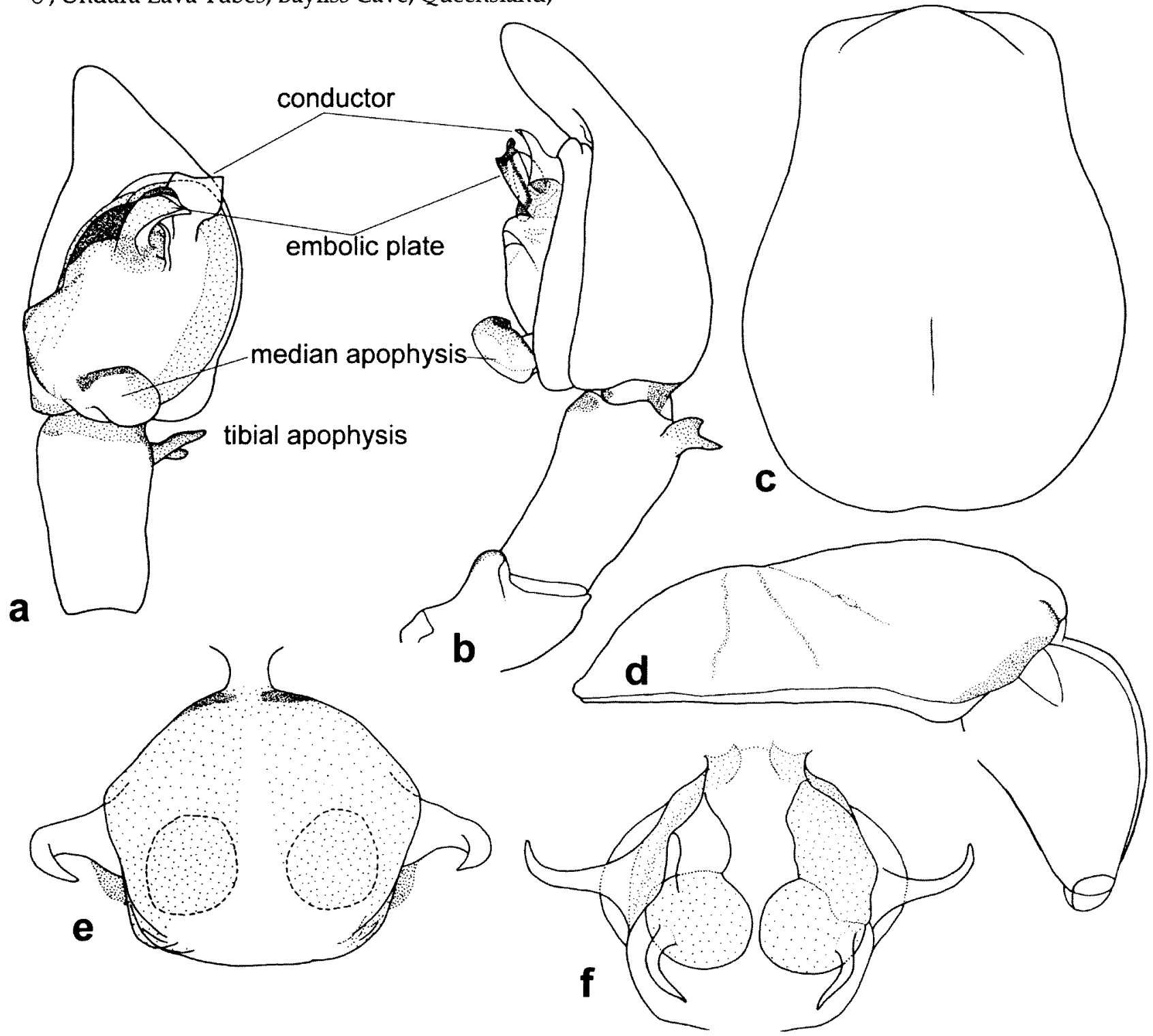

Figure 32 Amauropelma undara sp. nov. a, b, male, palpal tibia and tarsus, ventral view (a), with patella (b); $c-f$, female, c, d, carapace, dorsal view (c), with chelicera, lateral view (d); e, f, epigyne, ventral view (e), dorsal view (f). Scale lines $=0.5 \mathrm{~mm}$ for $\mathrm{a}, \mathrm{b}, 1 \mathrm{~mm}$ for $\mathrm{c}, \mathrm{d}, 0.27 \mathrm{~mm}$ for $\mathrm{e}, \mathrm{f}$. 

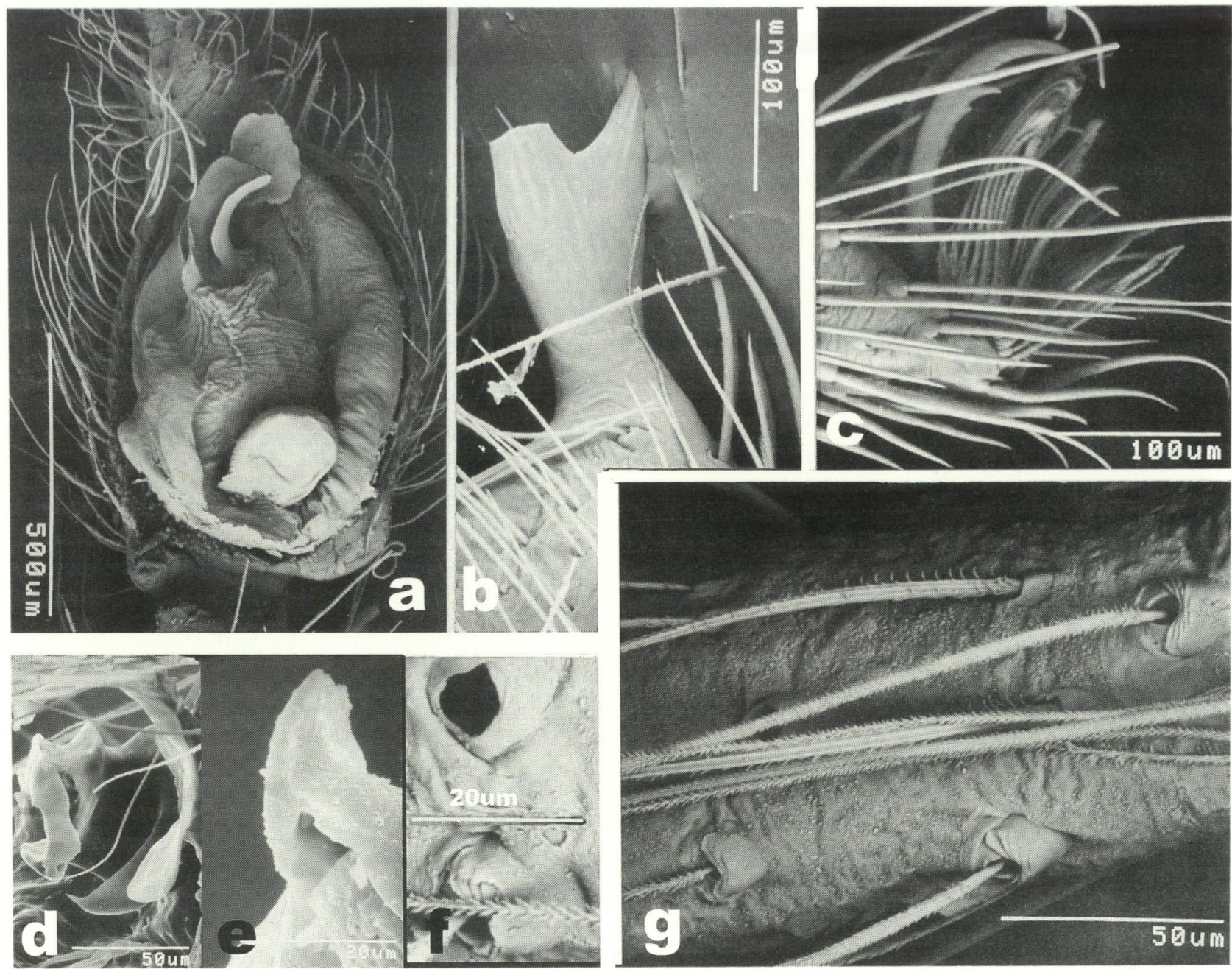

Figure 33 Amauropelma spp. A. undara sp. nov., a-c, e-g. d, A. hasenpuschi sp. nov.: scanning electron micrographs, male. Palp, ventral right: $a$, cymbium, bulb and distal tibia, ventral view; $b$, patellar apophysis, rotated retroventral view; c, distal tarsus I, lateral view; d, e, axial view of embolus showing spermatic opening; $f$, tarsal organ and trichobothrium, dorsal view; g, tarsus showing rugose surface and erect and adpressed trichobothria.

Table 15 Leg measurements of Amauropelma undara

Holotype male (QMS31389).

\begin{tabular}{llllll}
\hline & Leg 1 & Leg 2 & Leg 3 & Leg 4 & Palp \\
\hline Femur & 3.38 & 3.23 & 2.85 & 3.85 & 1.31 \\
Patella & 1.31 & 1.00 & 1.00 & 1.00 & 0.46 \\
Tibia & 4.15 & 3.31 & 2.85 & 4.23 & 0.46 \\
Metatarsus & 3.08 & 2.77 & 2.61 & 4.15 & \\
Tarsus & 1.61 & 1.31 & 1.15 & 1.77 & 1.00 \\
\hline Total & 13.53 & 11.62 & 10.46 & 15.00 & 3.23 \\
\hline
\end{tabular}

Allotype female (AMKS50693).

\begin{tabular}{lllll}
\hline & Leg 1 & Leg 2 & Leg 3 & Leg 4 \\
\hline Femur & 3.79 & 3.65 & 3.42 & 8.37 \\
Patella & 1.62 & 1.59 & 1.40 & 1.49 \\
Tibia & 3.80 & 3.55 & 3.20 & 4.46 \\
Metatarsus & 2.85 & 2.85 & 3.02 & 4.64 \\
Tarsus & 1.10 & 1.16 & 1.22 & 1.59 \\
\hline Total & 13.16 & 12.80 & 12.26 & 20.55 \\
\hline
\end{tabular}

\section{Diagnosis}

Differs from all other species in the absence of eyes.

\section{Description}

\section{Holotype male (QM S31389)}

Carapace 2.38 long, 1.85 wide. Abdomen 2.30, 1.58 wide.

Colour. Entirely pallid, only darker cuticle on anterior basal trochanters and palpal bulb. Carapace uniform light cover of moderately long brown bristles. One line of $4-6$ bristles arises across on clypeus. Fovea short in a deep groove begins just behind caput ends at edge of back slope. Eyes. Entirely absent with low domed mound where AME would be. Chelicerae. Promargin with 1 tooth; retromargin with 4 small. Sternum cordate with angles at intercoxae. Maxillae basally with 2 setae flanked by short shallow grooves. Legs. Elongate. Iridescent sheen on legs. No scopula, setation sparse. Tarsal organ low, at basal 1/3. Thick spine- 
like setae on ventral tarsi. Spines. As for genus except as noted. I: fe pv1p1. II: fe p1. III: fe p2d2 r1; pa 0; ti p2d1r2 v0.2.2; me p3r5v2.2.2. IV: fe p1d2 r1; pa 0; ti p2d1r2v2.2.0; me p5r5v2.2.2. Palp: fe p1d1.2; pa 0; ti 0. Claws. Elongate curved with 3-5 long teeth medially. Tufts small, distinct, no clear division. Trochanteral notches distinct, shallow, $2 x$ wider than deep. Trichobothria. One very long basally and medially on metatarsi. Palp. Patella with retroventral incision elevated as blunt flap. Tibial apophysis retrodorsal, a narrow process equally divided into 2 flattened triangular points (one broken on ventral right palp in SEM). Cymbium long, with conical apex uniformly setose, prolateral basal corner with blunt darkly sclerotised edge with triangular extension of tibia fitting prodorsal (larger) and proventral (smaller). Embolic plate a short wide grooved process set off tegulum by small translucent hand-shaped conductor. Median apophysis isolated, closed with round aperture. Tegular part of interlocking lobe with distinct point, in front (below) a triangular extension of less sclerotised tegular ring. Spinnerets. Short, clearly separated, ALS c.1.5 diameter apart and thicker than PLS but similar length. PMS short cylindrical.

\section{Allotype female (AM KS50693)}

Carapace 3.59 long, 2.56 wide. Abdomen 3.12, 2.15 wide.

Colour. Entirely yellowish without darker spots or bands. Carapace. Broad, uniformly hirsute; eye region a low mound for about 0.5 of head width; caputl ong, defined by shallow lateral grooves; fovea short. Chelicerae. Large, robust; 4 similar teeth on retromargin, promargin with 3 teeth, middle longest. Dark median zone basally of teeth in large pallid area enclosing basal teeth. Labium. Longer than wide, anteriorly indented. Maxillae. Longer than wide, about twice length of labium. Sternum. Narrowly cordate. Legs. Coxae with precoxal sclerites anteriorly and posteriorly. Large pallid retrocoxal hymen on I. All trochanters deeply notched. Preening combs absent. Scopula of long hairs distinct on metatarsi and tarsi I, II; absent on tibiae. Tarsal organ at distal $1 / 3$ on $\mathrm{I}, \mathrm{II}$, but at basal $1 / 4$ on III, IV.

Spines. As for genus except as noted. I: fe pv1p2d3r3. II: fe pv1p2d3r3. III: fe pv1p2d3r4; pa p1r1; ti p2d3r2; me p2r2pv1.1rv1.1v2.2.1. IV: fe pv1p2d2r2; pa p1r1; ti p2d3r2; me p3r3v1.1.1.2.2.2. Palp: fe fe p1d1.2r1; pa p1r1; $t$ p2d3r1; ta p2.1.1r3. Claws. Long, slender with 34 teeth just distal of midpoint. Claw tufts dense, separate, set on (?)erectible bases orthogonal to long tarsal axis. Spinnerets. ALS separated; PMS cylinders. Epigyne. A broad subquadrate rounded plate with long soft slender lateral horns; large receptaculum externally evident. An epigynal plug was removed from the anterior ectal corner.

\section{Distribution and Habitat}

Known only from caves the Undara lava tunnels via Townsville, north-eastern Queensland.

\section{Etymology}

A noun in apposition taken from the type locality.

\section{Amauropelma wallaman \\ Raven and Stumkat, sp. nov. \\ Figures 4, 28e, 34, Table 16}

\section{Material Examined}

\section{Holotype}

$\delta$, Wallaman Falls, via Ingham, Queensland, Australia, $18^{\circ} 36^{\prime} \mathrm{S}, 145^{\circ} 47^{\prime} \mathrm{E}$, rainforest, sieved litter, 1 October 1980, G. Monteith (QMS 32896).

\section{Allotype}

Australia: Queensland: 1 \%, Cardwell Gap, $18^{\circ} 31^{\prime} \mathrm{S}, 146^{\circ} 11^{\prime} \mathrm{E}$, pitfall, 24 September -25 November 1992, R. Raven, P. Lawless, E. Lawless, M. Shaw (QM S24891).

\section{Diagnosis}

Males differs from those of A. gayundah in that the median apophysis has a dominant lumen evident ventrally. Females differ from those of $A$. mossman in having the PME of similar size to other eyes.

Table 16 Leg measurements of Amauropelma wallaman.

Holotype male (QM S32896).

\begin{tabular}{llllll}
\hline & Leg 1 & Leg 2 & Leg 3 & Leg 4 & Palp \\
\hline Femur & 2.00 & 1.68 & 1.84 & 2.28 & 1.00 \\
Patella & 1.00 & 1.00 & 0.84 & 0.96 & 0.48 \\
Tibia & 1.80 & 1.28 & 1.24 & 1.80 & 0.48 \\
Metatarsus & 1.56 & 1.40 & 1.48 & 2.08 & \\
Tarsus & 0.84 & 0.80 & 1.28 & 1.28 & 1.04 \\
\hline Total & 7.20 & 6.16 & 6.24 & 8.40 & 3.00 \\
\hline
\end{tabular}

Allotype female (QM S24841).

\begin{tabular}{llllll}
\hline & Leg 1 & Leg 2 & Leg 3 & Leg 4 & Palp \\
\hline Femur & 1.92 & 1.68 & 1.60 & 2.04 & 0.88 \\
Patella & 0.92 & 0.92 & 0.80 & 0.92 & 0.52 \\
Tibia & 1.44 & 1.32 & 1.12 & 1.80 & 0.52 \\
Metatarsus & 1.16 & 1.08 & 1.20 & 1.92 & \\
Tarsus & 0.60 & 0.52 & 1.00 & 1.00 & 0.60 \\
\hline Total & 6.04 & 5.52 & 5.40 & 7.68 & 2.52 \\
\hline
\end{tabular}



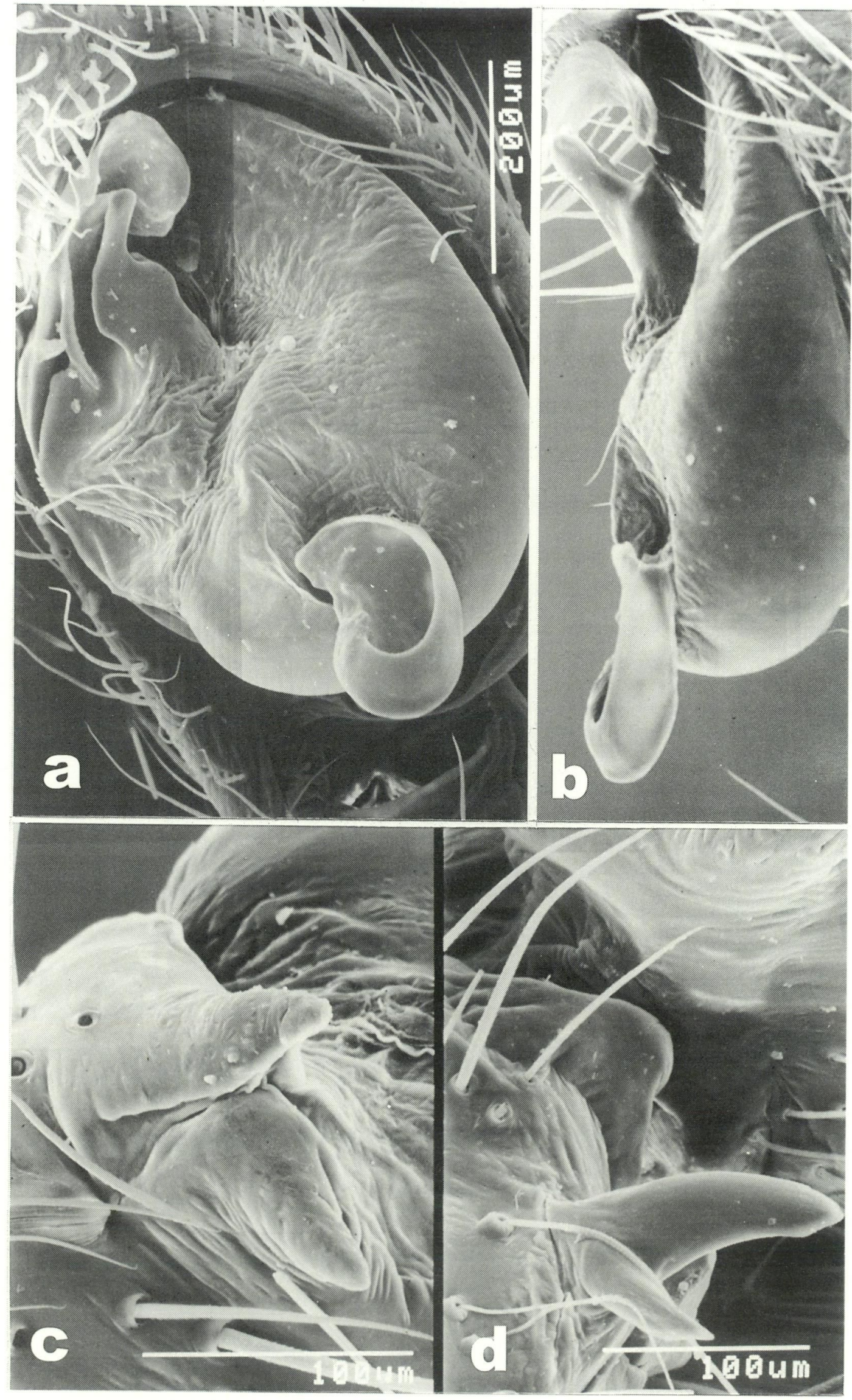

Figure 34 Amauropelma wallaman sp. nov.: scanning electron micrographs, male. Palp, ventral right: a, b, cymbium, bulb and distal tibia, ventral view (a), retrolateral view (b); c, patellar apophysis, retroventral view; d, tibial apophysis, retrolateral view. 


\section{Description}

Holotype male (QM S32896)

Carapace 2.44 long, 2.00 wide. Abdomen 2.00, 1.20 long.

Colour. Carapace light orange brown with narrow dark edge, dark lines along strial edges and on caput. Legs collapsed but yellow brown with slightly darker bands distally on femora and basally on tibiae. Abdomen pallid with dark areas laterally and distally forming paired long ovoid zones anteriorly and 3 transverse bars posteriorly. Carapace. Glabrous except for fine hairs along margins. Eyes. AME on common tubercle. ALE and PLE with bases of tubercle touching. Sinuous, loosely grate-shaped tapetum just evident. All eyes about equal in size. Spines. As for A. rifleck. Palp. Patella with two dissimilar small cones, ca. 1 diameter apart. Tibia with low retrodorsal mound with deeply divided tip; distal tip about 2-3 diameters of basal tip. Cymbium with short but distinct retrobasal process, and large probasal heel; no ventral spines on apical cone. Tegulum C-shaped but basally extends out in broadly rounded 'chin', median apophysis subovate with large extensive lumen and set basally on tegulum, longitudinal and set precariously on edge; conductor broadly flared, embolic plate with folded groove.

\section{Allotype female (QM S24891)}

Carapace 2.56 long, 1.84 wide. Abdomen 2.80 long, 1.92 wide. Total length 5.7 .

Colour. Carapace yellow brown with dark sashes laterally and dark margins. Abdomen colour lost. Legs yellow brown with mid- and distal bands on femora and tibiae, central bands on patellae and weakly on metatarsi. Eyes. Group width/head width $=0.64 ; \mathrm{PME}=10 \%$ of head width. Spines. As for genus except as noted. On tibia I, (ventral) spines are distally higher on lateral face than basally. I, II: fep1d3. III: fe pv1p2d3r1; pa p1r1; ti p2d3r2; me p2d2r2v2.2.2. IV: fe p2d3r1; pa r1; ti p2d2r2; me p2d2r2v2.2.2. Palp: fe p1d1.2; pa p1; ti p1d1; ta p3. Spinnerets. ALS with slightly enlarged spigot medially on tip. PMS with 2 enlarged spigots. Epigyne. A plate about 1.5 times wider than long with short soft lateral horns.

\section{Distribution and Habitat}

Known only from rainforest at and near Wallamans Falls, near Ingham, north-eastern Queensland.

\section{Etymology}

A noun in apposition taken from the type locality.

\section{ACKNOWLEDGEMENTS}

This paper is dedicated to Dr Glenn Hunt, 1944
1999. colleague, friend and highly esteemed scientist who inspired not only by his professional but also by his personal example of courage and dedication. We are very grateful to Dr Diana Silva, American Museum of Natural History, New York, for provocative discussions, comments on the manuscript and especially the suggestion to compare Amauropelma to Thoriosa Simon. Drs Barbara Baehr and V. Davies, Queensland Museum, provided support and counterpoint through the final phases. Mrs Helen Stark skillfully illustrated Amauropelma mossman (Figure 1) and Dr Barbara Baehr very kindly inked figures of epigynes and commented on the manuscript. Ms Wendy Rawlinson, Queensland Museum, very ably helped with registration and sorting of material. Dr Rudy Jocqué, Musée Royal de l'Afrique Centrale, Tervuren, Belgium, kindly loaned the material of Thoriosa spinivulva Simon (RMAC 131.124) and Thoriosa taurinain Simon (RMAC 131.101). For all of that, we are very grateful.

\section{REFERENCES}

Benoit, P.L.G. (1976). Etudes sur les Ctenidae africains (Araneae). II. Les genres Thoriosa Simon et Trogloctenus Lessert. Revue Zoologiques Africains 90: 221-227.

Davies, V. (1976). Spiders. In: Fauna of Eastern Australian Rainforests: preliminary report on sites surveyed by the Queensland Museum in mid-eastern and north-eastern Queensland. Queensland Museum, Brisbane.

Davies, V. (1977). Spiders. In: Fauna of Eastern Australian Rainforests II. Queensland Museum, Brisbane.

Davies, V. (1986). New Australian species of Otira Forster and Wilton, 1973 and Storenosoma Hogg, 1900 (Araneae, Amaurobiidae). Memoirs of the Queensland Museum 22: 237-251.

Dippenaar-Schoeman, A.S. and R. Jocqué (1997). African Spiders. An identification manual. Plant Protection Research Institute Handbook No. 9. Biosystematics Division, ARC-Plant Protection Research Institute, Ultra Litho, Johannesburg.

Forster, R.R., Wilton, C.L., 1973. The spiders of New Zealand. Part IV. Agelenidae, Stiphiidae, Amphinectidae, Neolanidae, Ctenidae, Psechridae. Otago Museum Bulletin 4: 1-309.

Gray, M.R. (1973). Cavernicolous spiders from the Nullarbor Plain and south-west Australia. Journal of the Australian Entomological Society 12: 207-221.

Griswold, C.E. (1991). A revision and phylogenetic analysis of the spider genus Machadonia (Araneae, Lycosoidea). Entomologia Scandanavica 22: 305-351.

Griswold, C.E. (1993). Investigations into the phylogeny of the lycosoid spiders and their kin (Arachnida, Araneae, Lycosoidea). Smithsonian Contributions to Zoology 539: 1-39.

Griswold, C.E. (1994). A revision and phylogenetic analysis of the spider genus Phanotea Simon (Araneae, Lycosoidea). Annales Sciences Zoologique 273: 3-63. 
Koch, L. (1878). Die Arachniden Australiens, nach der natur beschrieben und abgebildet. Vol. 1: 969-1064. Bauer and Raspe, Nürnberg.

Lehtinen, P.T. (1967). Classification of the cribellate spiders and some allied families, with notes on the evolution of the suborder Araneomorpha. Annales Zoologici Fennici 4: 199-467.

Platnick, N.I. 2000. A relimitation and revision of the Australasian ground spider family Lamponidae (Araneae: Gnaphosoidea). Bulletin of the American Musenm of Natural History 245: 1-330.

Rainbow, W.J. (1911). A census of Australian Araneidae. Records of the Australian Museum 9: 107-319.

Raven, R.J. (1981). A review of the Australian genera of the mygalomorph subfamily Diplurinae (Dipluridae: Chelicerata). Australian Journal of Zoology 29: 321-363.
Raven, R.J. (1994). Mygalomorph spiders of the Barychelidae in Australia and the western Pacific. Memoirs of the Queensland Museum 35: 291-706.

Raven, R.J. (1998). Revision of the Australian genera of the Miturgidae with a preview of their relationships. XIVth International Congress of Arachnology, Chicago, 1998. Abstracts: 31.

Thorell, T. (1881). Studi sui Ragni Malesi e Papuani. III. Ragni dell'Austro-Malesia o del Capo York, conservati nel Museo Civico di Storia Naturale di Genova. Annali del Museo Civico di Storia Naturale Giacomo Doria, Genova 17: 1-720.

Manuscript received 27 April 2000; accepted 21 December 2000. 


\section{CONFERENCE ABSTRACTS}

Papers presented to sessions on subterranean biology at the conference Dampier 300-Biodiversity in Australia 1699-1999 and beyond formed the background to this publication. The pertinent abstracts from this conference, with the addition of those pertaining to springs and karst issues, are given in the following section.

The conference was held in the Alexander Library Theatre, Perth Cultural Centre, Francis Street, Perth, Western Australia from 6 to 10 December 1999. It was organised by the Australian Systematic Botany Society, the Society of Australian Systematic Biologists and Invertebrate Biodiversity and Conservation in association with the Royal Society of Western Australia and the Western Australian Museum.

In the order presented:

Andrew Boulton: 'Twixt two worlds: surface water/groundwater interface.

George Wilson: Australian groundwater-dependent isopod crustaceans.

Brenton Knott and Edyta Jasinska: Insights into the fauna of the Gnangara Mound aquifer, Western Australia-the significance of springs.

Bill Humphreys: Groundwater calcrete aquifers in the Australian arid zone: an unfolding plethora of stygal diversity.

Mia Thurgate and Andy Spate: Biodiversity of the invertebrate fauna of caves in New South Wales.

Fred Stone and Frank Howarth: Why are there so many cave-adapted species in Australia? Relictual species versus the adaptive shift hypothesis.

Elery Hamilton-Smith and Stefan Eberhard: Maintenance of karst biodiversity.
Hannelore Hoch and Barbara Hosfeld [presented by Fred Stone]: Cave-adaptation in Australian planthoppers (Insecta: Hemiptera: Fulgoromorpha: Cixiidae): colonisation of novel habitat or response to climatic change?

David Slaney: Evolution of cave cockroaches in Australia. (not presented: an extended abstract of this work is provided below).

Bill Humphreys: Westralian Superbasin: is the tethyan connection supported by the extant anchialine community?

Stefan Eberhard: Managing and monitoring cave fauna in Tasmania.

Philippa Uwins, Richard Webb, Majid Ghoddusi, Anya Yago, Tom Loy and Kathleen Murphy: Novel nano-organisms (nanobes): living analogues for Martian 'nannobacteria'?

\section{POSTERS}

Niall Doran, Ian Houshold and Mike Driessen: Tasmanian cave guides: an under-used resource?

Travis Gotch: Spiders of the South Australian mound springs and bore drains.

David Slaney: Evolution of cave cockroaches in Australia.

Andy Spate, Jane Gough and Mia Thurgate: Karstic groundwater ecosystems in the Murray Darling and Otway groundwater basins.

Fred Stone: Distribution of surface and caveadapted cockroaches in the family Nocticolidae in Australia: evidence for evolution of tropical troglobites.

Mia Thurgate: Stromatolite biodiversity in the South-East of South Australia. 


\title{
'Twixt two worlds: surface water/groundwater interface
}

\author{
Andrew Boulton \\ Ecosystem Management, University of New England, Armidale, New South Wales 2350
}

Most of us are familiar with the taxonomic and functional biodiversity associated with the ecotones between two habitats, often resulting from an overlap of the biota and ecological processes that typify the two 'worlds that collide'. Two such worlds are the dark 'underworld' of groundwater and the well-lit surface environment of streams and rivers. 'Twixt these two contrasting worlds lies the hyporheic zone-the saturated sediments below the channel and extending out beneath the banks of many streams and rivers throughout the world. Surface stream water downwells into the hyporheic zone at the heads of riffles or the leading edges of central and lateral bars, and may travel underground for long distances (up to several kilometres) before emerging. Such upwelling water is usually altered chemically during its travels, and capable of influencing the composition of surface communities near its outflow. For example, in some streams, upwelling water may be enriched in nutrients that otherwise limit algal growth in the surface stream so that the upwelling zones are 'hotspots' for benthic algal production.

The hyporheic zone first attracted scientists' attention in European rivers during the early part of this century. Extraordinary creatures, many of them never seen in the surface waters, were described, often typified by morphological features interpreted as adaptations to a subterranean (stygal) existence: blind, unpigmented, elongate appendages, long sensory setae or antennae. This specialised fauna, termed the hyporheos, has now been found worldwide, living below nearly all rivers with a well-developed hyporheic zone. Diverse hyporheic assemblages occur from the unstable sandbeds of intermittent desert streams to the extensive glacial sediments of large lowland temperate rivers. Hyporheic invertebrates have been collected several kilometres from the main channel, and the magnitude of the hyporheic zone is sometimes an order of magnitude greater than the surface stream. Often, surface invertebrates temporarily occupy the hyporheic zone, apparently seeking refuge from droughts, floods or pollutants in the surface stream. Others, usually as small instars, may avoid predators or be actively entering the hyporheic zone to graze microbial biofilms on the sediment particles. Meanwhile, denizens typical of the saturated interstices of the deeper sediments near true groundwater are sometimes found quite close to the surface, carried there by upwelling water or perhaps actively migrating for some unknown reason.

In Australia, the hyporheos has been sampled in relatively few rivers. Biodiversity seems to be related to sediment particle size, water quality, and possibly food availability. In some places, the diversity of some groups (e.g. water mites) seems surprisingly high but as there are so few regional data, it is difficult to judge whether this is typical or not. Human activities, such as uncontrolled land clearance and consequent siltation, probably impact on the diversity of the hyporheos. It is likely that the 'health' of the hyporheic zone may be measured by hyporheic invertebrate biodiversity in similar ways to our assessment of river health using surface macro-invertebrate assemblage composition.

Trophic biodiversity in the hyporheic zone is biased towards detritivory and carnivory as the dark conditions minimise the amount of subterranean photosynthesis. In downwelling zones, some green plant material may be transported, but the main food and energy source appears to be derived from dead organic matter imported from the surface. Recent efforts to classify hyporheic invertebrates into 'functional groups' based on their diet and typical habitat have provided tools to predict responses to changes in physical features of the hyporheic zone or to hypothesise about the likely assemblages to be found in habitats of different particle size and hyporheic water residence time.

We have known of the existence of the hyporheos for nearly 80 years. The functional significance of the hyporheic zone has only become clear in the last 20 years or so. As we enter a new millenium with a clearer understanding of the linkages among streams, their catchments, their airsheds and the groundwater, we have a growing appreciation of the fundamental importance 
of the ecotones between these ecosystem compartments, and their taxonomic and trophic biodiversity. We need to preserve this biodiversity, already threatened by many of our activities but also at risk from our ignorance of its existence. As we change the conditions on either side of the hyporheic zone, what happens 'twixt the two worlds? And if we alter the exchanges of water, nutrients, energy, and fauna across this dynamic ecotone, what will be the ultimate fate of the two worlds that depend on the maintenance of this pathway?

\title{
Managing and monitoring cave fauna in Tasmania
}

\author{
Stefan Eberhard \\ Caveworks, P.O. Witchcliffe, Western Australia 6286
}

In Tasmania, cave management plans and monitoring programs recognise the significance of cave fauna, which is among the richest and most spectacular in temperate Australia. Conservation management of cave fauna has involved: (i) legislative protection of rare and threatened species; (ii) protection of sensitive habitats in caves by marking routes or no-go (sanctuary) areas; (iii) educating cave users, including minimum-impact caving techniques. Monitoring cave fauna has been undertaken for the purposes of: (i) gathering baseline ecological information for research, visitor management, and interpretation; (ii) measuring the ecological impacts of limestone quarrying and subsequent recovery during rehabilitation efforts.

In Kubla Khan Cave at Mole Creek, Tasmania, habitat degradation caused by cave visitors has been halted by installing a steel stairway. In Exit Cave at Ida Bay, certain representative or key habitat areas have been protected with string-line pathways, or else designated as no-go fauna sanctuary areas. The fauna sanctuaries include, inter alia, optimum habitat of the extremely rare blind cave beetle Goedetrechus mendumae. Although currently listed as vulnerable, this species is not considered threatened by trampling. A number of Tasmanian cave species are listed as rare or vulnerable, whilst others are wholly protected. The integrity of cave ecosystems at Mole Creek is most under threat from farm development on the karst, which affects the surface vegetation, water quality, flow regimes, nutrient and sediment inputs. Public awareness of cave fauna has been addressed by producing illustrated fact sheets which describe Tasmanian cave species, their vulnerable habitats, and minimum impact caving techniques.

The Little Trimmer Cave monitoring program was established by the Forestry Commission in 1991, to gather baseline environmental data. The biological monitoring, which has been continued by the University of Tasmania, has provided very useful life history and behavioural information on cave spiders, crickets and amphipods. Monitoring the abundance of stream-dwelling snails, as a potential indicator of quarrying impacts, commenced in Exit Cave in 1992. This monitoring is being continued in an attempt to gauge the effectiveness of rehabilitation efforts. The same quarrying operation caused extinction of the aquatic fauna in Bradley Chesterman Cave, but recovery of the fauna occurred within five years of quarry closure, after intensive rehabilitation measures.

Monitoring of glow-worms, cave crickets and cave spiders has been initiated in Exit Cave and Mystery Creek Cave to: (i) expand knowledge of the caves' biological resources; (ii) to identify environmental seasons, cycles, changes and trends; (iii) to provide a baseline upon which to assess the impact of human activity in the caves. However, obtaining statistical validity may be difficult when attempting to detect human impacts to cave fauna.

\section{REFERENCES}

Eberhard, S.M. (1995). Impact of a limestone quarry on aquatic cave fauna at Ida Bay in Tasmania. Proceedings of the 11th Australasian Cave and Karst Management Association Conference, Tasmania, May 1995. pp. 125-135. 
Eberhard, S.M. (1999). Cave fauna management and monitoring at Ida Bay, Tasmania. Tasmania Parks and Wildlife Service Nature Conservation Report No. 99/1.
Eberhard, S.M. (1999). Reconnaissance survey of cave fauna and management issues in the Mole Creek Karst National Park. Report to Parks and Wildlife Service, Tasmania.

\title{
Maintenance of karst biodiversity
}

\author{
Elery Hamilton-Smith ${ }^{1}$ and Stefan Eberhard ${ }^{2}$ \\ ${ }^{1}$ IUCN/WCPA Working Group on Cave and Karst Protection \\ ${ }^{2}$ Caveworks, PO Witchcliffe, W.A. 6286
}

\begin{abstract}
Biospeleology-the study of cave-dwelling organisms-has long been recognised as making a major contribution to the understanding of both evolutionary and adaptive processes and community ecology. A focus on the broader concept of the karst environment as a whole leads to consideration of a much wider range of inter-dependent organisms, ranging from microbiota, through a wide range of terrestrial, freshwater and anchialine invertebrates, various vertebrates, and karst-dependent plant associations adapted to life on alkaline soils, often with cyclic aridity.

This paper will review the Australian fauna, identify the major threats to its continuing biodiversity and discuss the importance of, and potential strategies for, protection. It will conclude by identifying major priorities for research and protective action.
\end{abstract}

\section{Cave-adaptation in Australian planthoppers (Insecta: Hemiptera: Fulgoromorpha: Cixiidae): colonisation of novel habitat or response to climatic change?}

\author{
Hannelore Hoch and Barbara Hosfeld \\ Presented by Fred Stone \\ Museum für Naturkunde, Humboldt-Universitet, Institut für Systematische Zoologie, \\ Invalidenstr. 43, D - 10115 Berlin, Germany
}

\begin{abstract}
The evolution of obligately cavernicolous (troglobitic) terrestrial organisms has been controversially discussed (relict versus adaptive shift hypotheses). The monophyletic cixiid genus Solonaima Kirkaldy, endemic in Australia (Queensland, New South Wales) provides an excellent model to test these hypotheses. At present, the monophylum contains eight epigean species (Queensland, New South Wales) and six cavernicolous species (Queensland). Epigean Solonaima species, which are largely known from museum collections only, have so far been recorded from localities along the continental divide, while cavernicolous species appear to be restricted to areas farther inland (Chillagoe Karst, Undara lava tubes) characterised by greater aridity. The cavernicolous species feed on tree roots (presumably Ficus spp.) which penetrate the cavernous rock. These roots provide a rich food resource for those organisms able to cope with high humidity, permanent darkness, and potentially high carbon dioxide concentrations. The cavernicolous Solonaima species represent several evolutionary lineages that have invaded caves in Queensland ranging from 5-10 million year old limestone caves to 190,000 year young lava tubes. The different lineages display varying degrees of troglomorphy, ranging from only slightly modified, troglophilic taxa to completely blind, flight- and pigment-less troglobitic species. Their
\end{abstract}


geographical distribution (areas with arid climate) indicates that climatic factors may have been the driving force for the evolution of cave-adapted taxa in Solonaima. It is conceivable that the desertification of the Australian continent during the Miocene has triggered a shift in behavioural or ecological strategies (e.g. food preference, choice of oviposition sites, larval development) that eventually resulted in cave-adaptation. In contrast, the degree of troglomorphy in the cavernicolous Solonaima species correlates strongly with the physical parameters (e.g. light availability, relative humidity, carbon dioxide concentration) of the cave environment where they are found rather than with the age of the caves or the availability of suitable food resource. These observations suggest that evolution of cave species in Solonaima may as well be an active adaptation to novel habitats.

Objectives of our present study are:

1. to reconstruct the phylogenetic relationships within the monophylum Solonaima;

2. to find a genetic marker that will allow us to calibrate speciation events against geological time.

In our analysis, we include previously uninvestigated character sets such as the ultrastructure of sensory organs, especially those located on the antennae and mouthparts, as well as configuration of leg spinulation and tarsal structure. These characters are expected to be of adaptive value during the colonisation of subterranean environments, as they may facilitate the insects' search for food and mating partners as well as enhance mobility on wet, rocky surfaces.

The results of our study are expected to contribute to better understand not only the evolutionary history of Solonaima but to the general processes underlying adaptation to high-stress environments.

\title{
Groundwater calcrete aquifers in the Australian arid zone: an unfolding plethora of stygal diversity
}

\author{
W.F. Humphreys \\ Western Australian Museum, Francis Street, Perth, Western Australia 6000
}

Present land that has ever been covered by the sea, even as far back as the Mesozoic, often supports communities of obligate subterranean aquatic species of marine lineages 'stranded' inland by marine recession, particularly in karstic areas. Large parts of Australia have not been inundated by the sea since at least the Palaeozoic, including the Pilbara and Yilgarn Cratons of Western Australia and their associated orogens, collectively referred to as the Western Shield. These Precambrian rocks covered by a thin regolith seem to be a poor prospect for the exploration of stygal biodiversity.

The Western Shield is incised by palaeodrainage systems dating from at least the Late Cretaceous, and with elements possibly dating from the Permian. A central watershed now separates the westerly drainages, some still active, from the largely inactive and disorganised inland drainages.

It was hypothesised that aquatic fauna associated with the palaeodrainage channels, with the onset of regional aridity, would progressively have become constrained to subterranean habitats and that the increasing salinity of the groundwater ultimately would have constrained the stygofauna to the still fresh headwaters of the palaeodrainage channels. As such, these isolated stygal communities should each contain a distinct fauna and thus contain substantial hidden biodiversity. Together these communities should provide information as to the history of these isolating events.

Sampling from the palaeodrainage systems has shown that the isolated freshwater bodies in the palaeodrainage systems contain distinct, even diverse, stygal communities and that they are isolated from others by intervening hypersaline water. The presumption that these isolated communities would be at the fresh headwaters of minor palaeodrainage channels was naive and the fauna is found within isolated groundwater calcrete bodies. It is not yet known whether the process by which this 
vicariance has occurred conforms with the hypothesis, but the stygofauna in these aquifers does represent substantial biodiversity. In addition, it has extended the salinity range of stygal habitat in continental groundwaters from oligohaline-limnic $\left(<5 \mathrm{~g} \mathrm{~L}^{-1}\right)$ to polyhaline $\left(18-30 \mathrm{~g} \mathrm{~L}^{-1}\right)$, possibly for the first time globally.

In the Australian arid zone, groundwater calcretes form within these palaeodrainage channels as a result of carbonate deposition due to evaporation from near surface groundwaters. They form in a particular part of a hydrogeological cycle that repeats itself along the length of the palaeodrainage lines, especially on the Western Shield. As a result, discrete calcrete bodies have formed along the length of the palaeodrainage channels, and some contain aquifers variously of fresh to hypersaline water. In places, karst processes within these calcretes have formed open conduit systems, thus providing habitat most suitable for subterranean fauna, both terrestrial and aquatic, while at the same time having properties ideal for water abstraction for human use, both attributes being sparse in the arid zone.

The calcrete aquifers contain subterranean aquatic faunas (stygofauna) that are both very diverse and often of a relictual nature. They include higher taxa new to Australia, amongst them Spelaeogriphacea, a Pangaean lineage now restricted to the hypogean zone of Gondwanan fragments and an order new to Australia, the genus Tiramideopsis (Acarina: Hydracarina) previously known from India, and Phreodrilidae (Oligochaeta). In addition, numerous species, largely in new genera, occur in the Bathynellidae (Syncarida), Copepoda (Cyclopoidea, Harpacticoidea, Calanoidea), Ostracoda (Candoninae), Amphipoda (Melitidae, Ceinidae, crangonyctoid), Isopoda (Phreatoicidea, Flabellifera and Oniscoidea) and Dytiscidae (Coleoptera).

\title{
Westralian Superbasin: is the tethyan connection supported by the extant anchialine community?
}

\author{
W.F. Humphreys \\ Western Australian Museum, Francis Street, Perth, Western Australia 6000
}

Anchialine systems are near coastal groundwaters that are affected by marine tides but having no surface connection to the sea. They have limited or no surface exposure, salinity stratified waters and typically are described from limestone or basalt caves. Anchialine systems typically contain phyletic and geographical relictual taxa and in recent years at least ten new families and even a new class (the Remipedia) of crustaceans have been described from anchialine caves, particularly on islands. The Cape Range region, northwestern Australia, has the only continental anchialine ecosystem known in the southern hemisphere.

A rich obligate subterranean aquatic fauna (stygofauna), principally Crustacea, occurs in the anchialine system of the Cape Range peninsula and Barrow Island, including the only southern hemisphere representatives of several higher level taxa. At least six lineages of Crustacea (Lasionectes, Remipedia; Halosbaena, Thermosbaenacea; Haptolana, Isopoda, Cirolanidae; Danielopolina, Ostracoda, Thaumatocyprididae; speleophriid and misophrioid copepods; pseudocyclopiid calanoid copepods; epacteriscid calanoid copepod; and possibly Hadziidae, Amphipoda) belong to genera or higher taxa whose general distribution pattern is coincident with the "Tethyan" track. Typically the global distributions of the genera (or higher taxon) is Cape Range, Lanzarote in the Canaries and the northern greater Caribbean region with some taxa in the Galapagos and Somalia.

This tethyan distribution pattern is generally considered to have formed in the epicontinental seas of the late Mesozoic, but this is long after the opening of the Atlantic. However, fossil evidence indicates that one lineage was already stygal in the Jurassic (leading to Danielopolina, Thaumatocyprididae), while in the extant species of Danielopolina and Halosbaena the Australian species is the sister group of those on either side of the Atlantic. Both sets of 
evidence are consistent with a Jurassic relictualisation of the Australian taxa and hence that the fauna characteristic of these anchialine systems spread westwards in the epicontinental seas formed by the development of Tethys into areas now the Mediterranean and Atlantic.

The persistence of anchialine ecosystems may occur because they are protected from surface vicissitudes both by being subterranean and by the possession of a stable ecotone associated with stratification of the water column. The anchialine systems are complex with strong physico-chemical stratification associated with thermohaloclines and involving multiple layers of hydrogen sulphide, anoxia and chemoautotrophic energy production probably associated with both nitrogen and sulphur bacteria. The typical 'tethyan' fauna largely occurs below the thermohalocline.

The geological context of the region is relevant. The Westralian Superbasin (Permian -Cretaceous) which straddled Australia and the formerly adjacent parts of Gondwana was epicontinental until late Mesozoic times. Fossil evidence suggests it was intermittently connected with Tethys. The only part of the superbasin remaining in the tropical Australian landmass is in the vicinity of the Cape Range peninsula, northwestern Australia, which has continuously been a shallow water marine environment since the Early Jurassic and which, since the Cretaceous, has been juxtaposed to the edge of the continental shelf in a marine carbonate depositional environment.

\title{
Evolutionary origins of cave cockroaches in the genus Paratemnopteryx Saussure (Blattodea: Blattellidae)
}

\author{
David Slaney \\ Department of Zoology and Tropical Ecology, James Cook University, Townsville, Queensland, Australia \\ present address: Health Research Centre, Department of Public Health, Wellington School of Medicine, \\ University of Otago, P.O. Box 7343, Wellington South, New Zealand
}

For over 150 years scientists have debated the origins of cave dwelling organisms. To investigate the origin of cavernicolous fauna in North Queensland, I selected the cockroach genus Paratemnopteryx Saussure (Blattaria, Blattellidae) to test alternative hypotheses for the evolution of cave dwelling species within the genus. The genus contains 13 described species, 11 of which are endemic to Australia, and five of which are cave dwelling (Roth, 1990; Slaney in press). Of the 13 described species three were recently collected and described, one surface dwelling species, $P$. rosensis, from southeast Queensland, and two cavernicolous species, $P$. kookabinnensis and $P$. weinsteini, from central Western Australia and northeast Queensland respectively (Slaney, in press). My initial hypothesis was that some surface dwelling species within this genus gave rise to cavernicolous species as a result of increasing aridity in Australia during the late Cenozoic. To test this, I used a combined morphological and molecular approach.

One hundred and sixty four Paratemnopteryx stonei and $P$. weinsteini adults were collected from seven caves in tropical North Queensland to investigate the degree of morphological modification, which may reflect various stages of adaptation to cave life. Morphometric studies, using canonical discriminant and cluster analyses, indicated morphological discontinuity between cave populations from the different geographic regions (Slaney and Weinstein, 1997). The body dimensions particularly important in discriminating among cave populations were tegmen length (both sexes), and secondly, tegmen width for males and tarsus length for females.

To reconstruct the phylogeny of the genus Paratemnoptery $x$ using a traditional morphological approach, 30 morphological characters were chosen, 11 binary and 19 multistate. The most informative sources of taxonomic characters were the male tergal glands, and the genitalia and associated structures for both sexes (Slaney, 1999). The resulting most parsimonious trees were consistent with previously proposed relationships within the genus.

In using a molecular approach to investigate relationships within the genus 
Paratemnopteryx, I sequenced the second internal transcribed spacer (ITS2) region of nuclear ribosomal DNA, and the entire mitochondrial cytochrome oxidase II gene (COII). Sequence data were obtained for nine Paratemnopteryx species and from several populations of some of those species. The size of the ITS2 gene region was $458-970$ base pairs, with a GC content of $50-58 \%$ (Slaney and Blair, 2000). The ITS2 data were useful for taxonomic discrimination at the population level, and supported the existence of at least three races of $P$. stonei, and of the new species $P$. weinsteini. However, the ITS2 region was too variable to be phylogenetically informative at the species level within the genus Paratemnopteryx. Analyses of the COII sequences from the same nine species, revealed a high AT bias, 71-72\% (Slaney, 1999). The majority of variable sites $(80 \%)$ occurred in the 3rd codon position, which contained most of the phylogenetic structure. The tree derived from maximum likelihood analysis of all sites in the COII gene was more similar with the morphological derived tree than was the parsimony based one (Slaney, 1999).

Cave colonisation events were traced on to the reconstructed phylogeny for Paratemnopteryx species to determine whether single or multiple colonisation(s) occurred. Results indicated that cave colonisation events occurred independently on at least three occasions, once in Western Australia and twice in North Queensland (Slaney, 1999).

In placing Paratemnopteryx species into seven main geographical regions covering continental Australia, two vicariance events are postulated (Slaney, 1999). One between northwest Western Australia and central Australia, the second in North Queensland. On a finer geographical scale, most species have restricted distributions with no overlap, and on this basis seven additional vicariance events may be postulated. Identification of these vicariance events involves mainly comparisons of cave dwelling species distributions, where specimens have only been found in certain karst regions. Investigation of the mode of speciation in the genus Paratemnopteryx supports allopatric speciation, with little or no subsequent range changes (Slaney, 1999). Analyses also indicate recent allopatric speciation of the cave dwelling species in North Queensland.

Evidence from species distributions, the structure of the phylogenetic trees, and the possible timings of cave colonisation events (calculated from the maximum likelihood analysis), support the Pleistocene-effect hypothesis for the evolutionary origin of cave cockroaches in the genus Paratemnopteryx (Slaney, 1999). Increasing and spreading aridity over much of Australia in the late Cenozoic may have provided strong selective pressures for surface dwelling species to colonise caves in search of moisture refugia. It is not clear if the present cavernicolous species became distinct from ancestral populations before or after entering caves, although it appears that $P$. stonei and $P$. howarthi may have done so well before colonising caves.

\section{REFERENCES}

Roth, L.M. (1990). A Revision of the Australian Parcoblattini (Blattaria: Blattellidae: Blattellinae). Memoirs of the Queensland Museum 28: 531-596.

Slaney, D.P. (1999). Evolutionary origins of cave cockroaches in the genus Paratemnopteryx Saussure (Blattodea: Blattellidae). PhD Thesis, James Cook University.

Slaney, D.P. (In press). New species of Australian cockroaches in the genus Paratemnopteryx Saussure (Blattaria, Blattellidae, Blattellinae), with implications for the evolution of tropical cave faunae. Journal of Natural History, London.
Slaney, D.P. and Blair, D. (2000). Morphology and molecules are concordant in discriminating among populations of cave cockroaches Paratemnopteryx Saussure (Blattaria: Blattellidae). The Annals of the Entomological Society of America 93: 398-404.

Slaney, D.P. and Weinstein, P. (1997). Geographical variation in the tropical cave cockroach Paratemnopteryx stonei Roth (Blattodea: Blattellidae) in tropical Queensland, Australia. International Journal of Speleology 25: 1-14. 


\title{
Biodiversity of New South Wales subterranean fauna
}

\author{
Mia Thurgate ${ }^{1}$ and Andy Spate ${ }^{2}$ \\ ${ }^{1}$ Jenolan Caves Trust, PO Box 1495, Bathurst, New South Wales 2795 \\ ${ }^{2}$ NSW National Parks and Wildlife Service, P O Box 2115, Queanbeyan, New South Wales 2620
}

New South Wales has some 450 carbonate rock outcrops of which 95 are known to be cavernous. Well over 2200 individual caves are known in these karst environments. In 1994/95, Eberhard and Spate (1995) completed a major survey of cave macro-invertebrate fauna from over 130 caves. More than 1200 specimens from at least 172 taxa were collected. The results of this survey significantly increased the known subterranean fauna of NSW. Compared with the state of knowledge published prior to 1993, the number of orders increased by nearly a third (from 21 to 30 ), and the number of families recorded was almost doubled (from 61 to 115). An increase in richness was evident amongst cave-limited genera as well. Before 1993, 11 troglobitic species had been recorded, and this has since increased to between 17 and 32 genera. The survey also revealed a previously unsuspected diversity of aquatic taxa.

At the level of individual karst areas, the overall richness of NSW areas is typically 10-25 species, considerably lower than for karst areas in Tasmania and Western Australia. However, several karst areas in NSW have a much higher level of richness. Some of the most notable sites include Jenolan (67 taxa), Wombeyan (58 taxa) and Wee Jasper (54 taxa). These sites also contain the highest number of troglobitic taxa in the State, including a diverse aquatic crustacean fauna. The richness of these sites may be due in part to their large size, topographic relief, and highly cavernous structure containing a wide variety of habitats. They have probably been maintained as stable, moist refugia (especially the permanent aquifers) over a geologically significant period, facilitating the survival of relictual populations.

Since the initial reporting of results of the Eberhard and Spate survey, several other locations have been flagged as important centres of biodiversity for subterranean biota. The Macleay Valley (west of Kempsey), includes a number of scattered karst localities with high species richness including Stockyard Creek (74 taxa) and Willi Willi (61 taxa). The Valley is also particularly important for its highly diverse terrestrial calciophilic gastropod assemblages. The Wellington karst aquifer and possibly the Bell River alluvial aquifers have also proved to be particularly important for their diverse, endemic aquatic invertebrate fauna. One taxonomic group, the division Syncarida, is particularly well represented in the Wellington/Bell River aquifer systems and across New South Wales generally, with a new family and two new genera represented by eight new species, currently being described.

As custodians of Jenolan, Wombeyan, Abercrombie and Borenore Karst Conservation Reserves, the Jenolan Caves Reserve Trust responsible for the conservation of several of the more significant subterranean biota sites in NSW. Jenolan Caves receive one of the highest cave visitations in the State, and there is concern that potentially high levels of impacts may occur in the show cave systems, in the more popular wild caves, and in the karst aquifer. In response to these potential threats, the Trust has formed a Visitor Impact Management system, which is overseen by the Social and Environmental Monitoring Committee. This Committee has given high priority to the maintenance of subterranean fauna populations and their habitats. Currently the Committee, along with the Trust's Karst Resources Department, is developing suitable survey methods for subterranean fauna collection, with a view to developing a long-term monitoring program.

This paper provides an overview of the current knowledge of the diversity of the subterranean biota of New South Wales. The more important karst regions and their taxa are described in detail, updating the Eberhard and Spate report (1995). The paper highlights several of the more important taxonomic groups including the amphipod, syncarid and gastropod faunas. Gaps in the current knowledge and suggestions for further research are outlined. Management implications for NSW karst areas, focusing on the Jenolan Caves Reserve Trust sites and Wellington Caves, are discussed. 


\title{
REFERENCE
}

Eberhard, S. and Spate, A.P. (1995). Cave Invertebrate Survey: Towards an Atlas of New South Wales Cave Fauna. Report to the Department of Urban Affairs and
Planning. NSW Heritage Assistance Program NEP 94 765. $120 \mathrm{pp}$.

\section{Novel nano-organisms (nanobes): living analogues for Martian 'nannobacteria'?}

\author{
Philippa J.R. Uwins ${ }^{1}$, Richard I. Webb ${ }^{1,2}$, Majid Ghoddusi ${ }^{1}$, Anya J.E. Yago ${ }^{1}$, Tom Loy ${ }^{3,4}$ \\ and Kathleen Murphy ${ }^{3,5}$ \\ ${ }^{1}$ Centre for Microscopy and Microanalysis, ${ }^{2}$ Department of Microbiology and Parasitology, ${ }^{3}$ Centre for Molecular and \\ Cell Biology, ${ }^{4}$ Department of Anthropology and Sociology, ${ }^{5}$ Queensland Agricultural Biotechnology Centre, \\ The University of Queensland, St Lucia, Queensland 4072
}

\begin{abstract}
On August 7 1996, NASA announced that they had found evidence that a Martian meteorite (ALH84001) contained evidence for past life on Mars (McKay et al., 1996). At the centre of their thesis, and perhaps the most controversial of their findings, were the fossil remnants of sub-bacteria sized 'cells' called nannobacteria. Whilst the findings were of great scientific interest they fuelled a fierce, highly contentious debate concerning the minimum size of autonomous life on earth.

The identification and existence of nannobacteria and nanobacteria was not new and had been proposed previously by a number of workers (Folk, 1993; Sillitoe et al., 1996; Pedone and Folk, 1996; Vasconcelos and Mckenzie, 1997; Folk and Lynch, 1997; Kajander et al., 1994; Kajander et al., 1998). The existence of nan(n)obacteria, however, has been fiercely questioned, since it is argued that the minimum size of autonomous life is $200 \mathrm{~nm}$ in diameter for a spherical cell (Vogel, 1998). It is thought that any organism smaller than 200 $\mathrm{nm}$ in diameter would have insufficient volume to conduct the metabolic and reproductive processes essential to live (Maniloff, 1997; Nealson, 1997; Psenner and Loferer, 1997; Vogel, 1998). It therefore remained unresolved whether 'nannobacteria' are fossilised remnants of autonomous life-forms, artefacts of sample preparation or unusual mineral deposits.

More recently, novel nano-organisms (nanobes) have been described growing on Triassic and Jurassic sandstones collected from petroleum exploration boreholes off the Western Australian coast (Uwins et al., 1998). These cells (20-150 nm in diameter) provide further evidence in strong support of nano-scopic life. For example, analysis of these growths shows that nanobes are communicable and grow; are composed predominantly of carbon, oxygen and nitrogen; contain DNA and RNA; and are membranebound structures surrounding a dense cytoplasm and nuclear area. The morphology of these growths closely resemble Actinomycetes and fungi, only on a much reduced scale. It is difficult to explain what these organic features are if they are not living cells, given that they are not composed of mineral compounds (silicates, sulphides or metal oxides), or carbonaceous compounds (e.g. fullerenes, carbon nanotubes or carbonates), nor can they be explained as non-living polymers.

The morphology, elemental composition, and ultrastructural detail of nanobes will be described, illustrated and discussed in the context of the nan(n)obacterial debate. It is our thesis that nanobes are living biological organisms in the same size range as the controversial nan(n)obacteria found in a range of different rock types, in biological tissues and in Martian Meteorite ALH84001.
\end{abstract}

\section{REFERENCES}

Folk, R.L. (1993). Nannobacteria in carbonate sediments and rocks. Journal of Sedimentary Petrology 63: 990999.
Folk, R.L. and Lynch, F.L. (1997). The possible role of nannobacteria (dwarf bacteria) in clay mineral diagenesis and the importance of careful sample 
preparation in high-magnification SEM study. Journal of Sedimentary Research 67: 583-589.

Kajander, E.O. and Ciftcioglu, N. (1998). Nanobacteria: An alternative mechanism for pathogenic intra- and extra-cellular calcification and stone formation. Proceedings of the National Academy of Sciences USA 95: 8274-8279.

Kajander, E.O., Tahvanninen, E., Kuronen, I. and Ciftcioglu, N. (1994). Comparison of staphylococci and novel bacteria-like particles from blood. Zentralblatt fur Bakteriologie 26: 147-149.

Maniloff, J. (1997). Nannobacteria: size limits and evidence. Science 276: 1776.

McKay, D.S., Gibson, E.K., Thomas-Keprta, K.L., Vali, H., Romanek, C.S., Clemett, S.J., Chillier, X.D.F., Maechling, C.R. and Zare, R.N. (1996). Search for past life on Mars: possible relic biogenic activity in martian meteorite ALH84001. Science 273: 924-930.

Nealson, K.H. (1997). Nannobacteria: size limits and evidence. Science 276: 1776.
Pedone, V.A. and Folk, R.L. (1996). Formation of aragonite cement by nannobacteria in the Great Salt Lake Utah. Geology 24: 763-765.

Psenner, R. and Loferer, M. (1997). Nannobacteria: size limits and evidence. Science 276: 1776-1777.

Sillitoe R.H., Folk, R.L. and Saric, N. (1996). Bacteria as mediators of copper sulfide enrichment during weathering. Science 272: 1153-1155.

Uwins, P.J.R., Webb, R.I. and Taylor, A. (1998). Novel nano-organisms from Australian sandstones. American Mineralogist 83: 1541-1550.

Vasconcelos, C. and McKenzie, J. (1997). Microbial mediation of modern dolomite precipitation and diagenesis under anoxic conditions (Lagoa Vermelha, Rio De Janeiro, Brazil). Journal of Sedimentary Research 67: 378-390.

Vogel, G. (1998). Finding Life's Limits. Science 282: 1399.

\title{
Australian groundwater-dependent isopod crustaceans
}

\author{
George D.F. Wilson \\ Centre for Evolutionary Research, Australian Museum, 6 College Street, Sydney, New South Wales 2000
}

This paper reviews the phylogenetic history, biogeography and diversity of isopod crustaceans that depend on Australian groundwater resources (AGDI). These isopods belong to two suborders, Phreatoicidea and Asellota. Other, more recently evolved isopods are present in Australian groundwaters (e.g. Cirolanidae), but in much lower frequencies.

1. Phylogenetically, AGDI are ancient, with either fossils or indirect phylogenetic information leading to presumptive ages from the late Palaeozoic to the early Mesozoic Eras. Palaeozoic marine fossils and Triassic freshwater Australian fossils establish the antiquity of Phreatoicidea. Peracaridan crustacean cladograms show the Phreatoicidea to be the earliestderived group of all isopods. In these same cladograms, the Asellota are either a sister group of the Phreatoicidea, or derived one branch later. Although Asellota do not fossilise, this phylogenetic pattern is consistent with a Palaeozoic origin of this suborder. This conclusion is also supported by a Gondwanan biogeographical pattern for both suborders. Sister taxa (related genera) of Australian genera are found in other Gondwanan fragments, providing a minimum age of approximately 180 million years for these taxa. A recent Northern Territory discovery of species belonging to the asellotan family Protojaniridae, known previously from South Africa and Sri Lanka, establishes another Gondwanan pattern. A much later-derived asellotan genus, Heterias, is also found in South America, providing another ancient Gondwanan connection. Heterias, however, belongs to a poorly defined family that includes marine, brackish and freshwater taxa, so a marine origin for Australian Heterias cannot be discounted.

2. AGDI may be classified into three ecotypes: surface-cryptic, surfaceburrower, and groundwater forms. The phylogenetic distribution of these ecotypes in phreatoicidean isopods provides evidence for at least two separate colonisations of groundwater habitats. One colonisation event may be older than the rifting of the Indian subcontinent and the other is dated approximately by the separation of New Zealand from East Gondwana. Although phylogenetic results are not available for the Asellota, both surfacecryptic and groundwater ecotypes are common. Freshwater Asellota do not burrow, but live either interstitially or in the burrows of other animals (pholeteros). 
3. Species and genera of AGDI are highly endemic. Single distinctive genera of Phreatoicidea occur in small areas of Australia. For example, the distinctive genus Phreatomerus occurs only in artesian mound springs of South Australia. Several new genera found recently are restricted to extremely small sites such as single groundwater-fed streams in the north-west Kimberley. In some cases, the regions have been sampled extensively, so the tiny distributions are not artifactual. We can say little about the Protojaniridae species, since only one Australian site is known and its taxonomic affinities have not been established. According to recent genetic studies, Heterias may be highly endemic at the species level.

4. Some regions in Australia harbour flocks of AGDI species. The currently monotypic genus Eophreatoicus Nicholls, 1943, found in Kakadu National Park and Arnhem Land (Northern Territory), contains 14+ undescribed species. Crenoicus has two or three undescribed species in each large watershed region in New South Wales above $1000 \mathrm{~m}$ (e.g. Kosciusko Plateau, Boyd Plateau, Barrington Tops), possibly reaching a total of 14-16 species in this State alone. Tasmania is replete with species flocks in several genera, especially in Colubotelson. The prevalence of species flocks of AGDI means that these taxa may add considerably to regional groundwater biodiversity assessments.

Taken together, these observations confer a high conservation value on AGDI, especially when using phylogenetic diversity models. Detailed systematics, phylogenies and biogeographical patterns thus have important roles in assisting informed decisions on the conservation of these and other invertebrate taxa.

\title{
ABSTRACTS OF POSTERS
}

\section{Species abundance and diversity of aquatic invertebrates in South Australian mound springs and bore drains}

\author{
Jason Cody, Andy Austin, John Jennings and Hugh Possingham \\ Department of Applied and Molecular Ecology, Waite Campus, The University of Adelaide, PMB 1, Glen Osmond,
} South Australia 5064

\begin{abstract}
The artesian mound springs found in the north-east of South Australia are of special ecological interest because they are the only source of relatively fresh, permanent free-water. Until recently, these were used extensively as water points for cattle, although some now have stock excluded. Since European settlement bores have also been sunk in the area to provide water for both humans and stock. Mound springs and bore drains provide a habitat for a number of invertebrate species in an otherwise arid environment. Some invertebrates (e.g. the isopod Phreatomerus latipes, the ostracod Ngarawa dirga, a phreatic amphipod Phreatochiltonia anophthalma, and amphipods of the genus Austrochiltonia.) appear to be endemic to the mound springs, whilst other non-endemic invertebrates (e.g. chironomids and aquatic beetles) make use of the free-water from bores. The species diversity of both mound springs and bores appears to be affected by the stocking rate imposed upon them. As well, a difference in the species composition between mound springs and bores is apparent. These apparent differences will be examined by comparing the species diversity of a number of springs and bores with similar stocking histories. Initial results suggest there is a general decline in the species diversity of mound springs after de-stocking, although the endemic species diversity appears unaffected. There also appears to be differences in the species composition between the mound springs and bores.
\end{abstract}




\title{
Tasmanian cave guides: an under-used resource?
}

\author{
Niall Doran, Ian Houshold and Mike Driessen \\ Nature Conservation Branch, Tasmanian Parks and Wildlife Service, GPO Box 44A, Hobart, Tasmania 7001
}

\begin{abstract}
The enthusiasm of cave guides and Parks and Wildlife field staff in Tasmania potentially offers new opportunities in the ongoing study of invertebrate cave fauna in the State. These opportunities may augment the wealth of taxonomic information gained through cave surveys and fauna collections conducted across Tasmania in the past, as well as the steadily building ecological database produced via established and new long-term monitoring programs (addressing both research and conservation management issues). While such surveys and programs have provided us with a great deal of information on Tasmanian cave fauna, they are commonly restricted to single or few collecting events or to surveys spaced a month (or more!) apart.

Guides employed by the Tasmanian Parks and Wildlife Service make multiple visits, totalling several hours per day, to tourist caves every day. These visits promote an intimate understanding of the caves in which they work and offer monitoring opportunities at a frequency that most scientific programs would envy. At the same time, the field guides are increasingly interested in information on cave fauna and the latest research developments in the subterranean world. At their instigation, we are currently helping to develop monitoring protocols that can be readily incorporated into the regular every day schedules of cave tours without adding significantly to their workload.

We are able to provide the guides with background information on cave fauna and physical karst processes (including, importantly, what is not known or recorded) and, with help in the design and analysis of monitoring programs and data, to ensure that it is in a form valuable to the science. In turn, the guides are able to return quantitative and qualitative data from both observations and surveys, covering events and behaviours that have otherwise only rarely been seen, and providing information that may fill the gaps in larger scale survey programs. It is hoped that this information will not only be of general interest, but may be incorporated in planned studies of the effect of different levels of disturbance on cave environments and invertebrate fauna. Involving the cave guides in this process will hopefully also benefit their ability to relate information directly to a public which itself shows a growing appreciation for the scientific and ecological aspects of cave formation and life. In future, it is hoped that the guides will not only continue relaying the latest information on cave fauna to the public, but will also relay increasing amounts of information that they themselves have been involved in collecting.
\end{abstract}

\section{Spiders of the South Australian mound springs and bore drains}

\author{
Travis Gotch, Andy Austin, Hugh Possingham, John Jennings and Drew Tyre \\ Department of Applied and Molecular Ecology, Waite Campus, The University of Adelaide, PMB 1, Glen Osmond, \\ South Australia 5064
}

The region to the south of Lake Eyre is one of the driest areas in Australia. The only natural permanent potable water in this region is from a series of discontinuous mound springs and pastoral bores that are clustered around the south-western edge of the Great Artesian Basin, giving them an importance that is out of proportion to the area they actually cover. The springs and bore drains can be considered to be analogous to islands as they are wetlands isolated by desert, effectively limiting the dispersal of some 
species. Recent research has identified 10 undescribed species of spider that depend on water. Some of these spiders are putatively endemic to the Lake Eyre complex of mound springs, possibly even to individual springs. The Lycosidae make up half of these spiders, the other five species are members of four other families, the Pisauridae, Hahniidae, Clubionidae, and Linyphiidae (two species). This poster outlines research currently under way to 1) identify which of the spider species are restricted to mound springs and which have successfully dispersed into bore drains and 2) identify the microhabitats of the springs and bore drains that are being utilised by the spiders.

\title{
Karstic groundwater ecosystems in the Murray Darling and Otway Groundwater Basins
}

\author{
Andy Spate', Jane Gough ${ }^{1}$ and Mia Thurgate ${ }^{2}$ \\ ${ }^{1}$ NSW National Parks and Wildlife Service, P O Box 2115, Queanbeyan, New South Wales 2620 \\ ${ }^{2}$ Jenolan Caves Trust, PO Box 1495, Bathurst, New South Wales 2795
}

Small, impounded, karstic aquifers are found widely on the western fall of the Eastern Highlands. Many of the aquifers support highly significant, but little studied, invertebrate faunas. Based on geological considerations, the Otway Groundwater Basin is part of the Murray Darling Groundwater Basin. In the Otway Basin, around Mount Gambier and to the southern coast, there are very many groundwater dependent ecosystems evident. Nothing is known about possible ecosystems in the Murray Group limestones underlying the Late Miocene to Quaternary sediments in the lower parts of the Basin.

This poster discusses aquatic karst ecosystems within the impounded karsts of the N.S.W. portion of the Murray-Darling Basin and the major karst province of the Otway Basin. These ecosystems are significant as centres of biodiversity for extant stromatolite communities and for subterranean fauna. Freshwater amphipod and syncarid faunas are particularly diverse within the karst groundwaters of these ecosystems. The Wellington Caves aquifer in particular has many values including a significant and diverse, endemic aquatic invertebrate fauna and is considered worthy of recognition as a RAMSAR site. Some potential threats to these important ecosystems are identified in this poster, and some potential research directions are canvassed. The poster also briefly discusses the presence of hyporheic systems. 


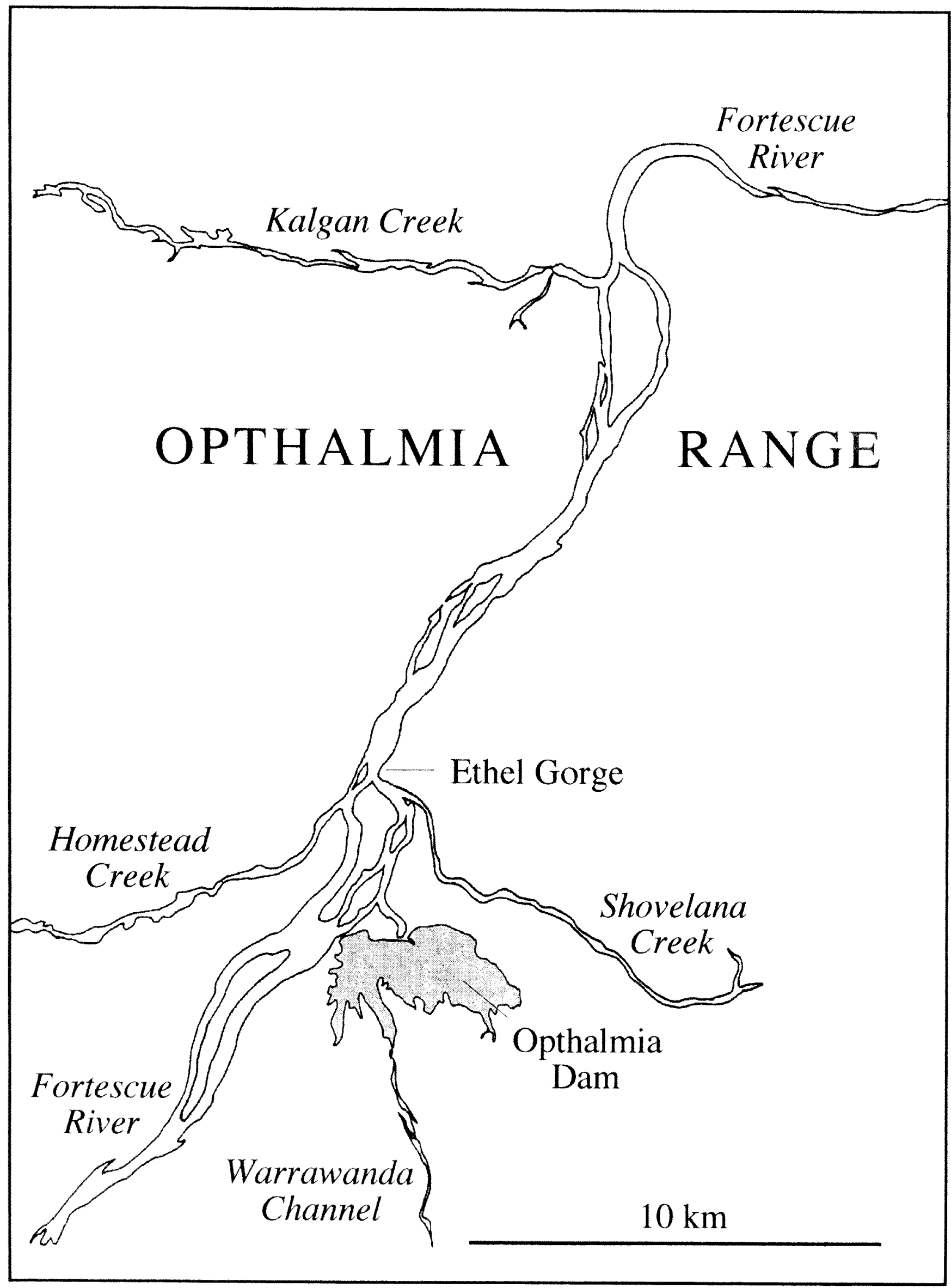




\section{Guide to Authors}

\section{Subject Matter:}

Reviews, observations and results of research into all branches of natural science and human studies will be considered for publication. However, emphasis is placed on studies pertaining to Western Australia. Longer papers will be considered for publication as a Supplement to the Records of the Western Australian Museum. Short communications should not normally exceed three typed pages and this category of paper is intended to accommodate observations, results or new records of significance, that otherwise might not get into the literature, or for which there is a particular urgency for publication. All material must be original and not have been published elsewhere.

\section{Presentation:}

Authors are advised to follow the layout and style in the most recent issue of the Records of the Western Australian Museum including headings, tables, illustrations and references.

The title should be concise, informative and contain key words necessary for retrieval by modern searching techniques. An abridged title (not exceeding 50 letter spaces) should be included for use as a running head.

An abstract must be given in full length papers but not short communications, summarizing the scope of the work and principal findings. It should normally not exceed $2 \%$ of the paper and should be suitable for reprinting in reference periodicals.

The International System of units should be used.

Numbers should be spelled out from one to nine in descriptive text; figures used for 10 or more. For associated groups, figures should be used consistently, e.g. 5 to 10 , not five to 10 .

Spelling should follow the Concise Oxford Dictionary.

Systematic papers must conform with the International Codes of Botanical and Zoological Nomenclature and, as far as possible, with their recommendations.

Synonymies should be given in the short form (taxon, author, date, page) and the full reference cited at the end of the paper. All citations, including those associated with scientific names, must be included in the references.

\section{Manuscripts:}

The original and two copies of manuscripts and figures should be submitted to the Editors, c/Publications Department, Western Australian Museum, Francis Street, Perth, Western Australia 6000. They must be in double-spaced typescript on A4 sheets. All margins should be at least $30 \mathrm{~mm}$ wide. Tables plus heading and legends to illustrations should be typed on separate pages. The desired position for insertion of tables and illustrations in the text should be indicated in pencil. Tables should be numbered consecutively, have headings which make them understandable without reference to the text, and be referred to in the text.

High quality illustrations are required to size $(16.8 \mathrm{~cm} \times 25.2 \mathrm{~cm})$ or no larger than $32 \mathrm{~cm} \times 40$ $\mathrm{cm}$ with sans serif lettering suitable for reduction to size. Photographs must be good quality black and white prints, not exceeding $16.8 \mathrm{~cm} \times 25.2 \mathrm{~cm}$. Scale must be indicated on illustrations. All maps, line drawings, photographs and graphs, should be numbered in sequence and referred to as Figure/s in the text and captions. Each must have a brief, fully explanatory caption. On acceptance a computer disk containing all corrections should be sent with amended manuscript. The disk should be marked with program (e.g. Word, WordPerfect, etc).

In papers dealing with historical subjects references may be cited as footnotes. In all other papers references must be cited in the text by author and date and all must be listed alphabetically at the end of the paper. The names of journals are to be given in full.

\section{Processing:}

Papers and short communications are reviewed by at least two referees and acceptance or rejection is then decided by the editors.

The senior author is sent one set of page proofs which must be returned promptly.

The senior author will receive fifty free offprints of the paper. Additional offprints can be ordered at page proof stage.

Back cover: Satellite image of the Fortescue River at Ethel Gorge, Pilbara. The blue expanse is Opthalmia Dam. Immediately upstream (lower left) of the gorge is a groundwater fauna, largely in calcrete, that includes the most species of stygal amphipods described from a single system globally. Features and scale are depicted on the end flyleaf. 


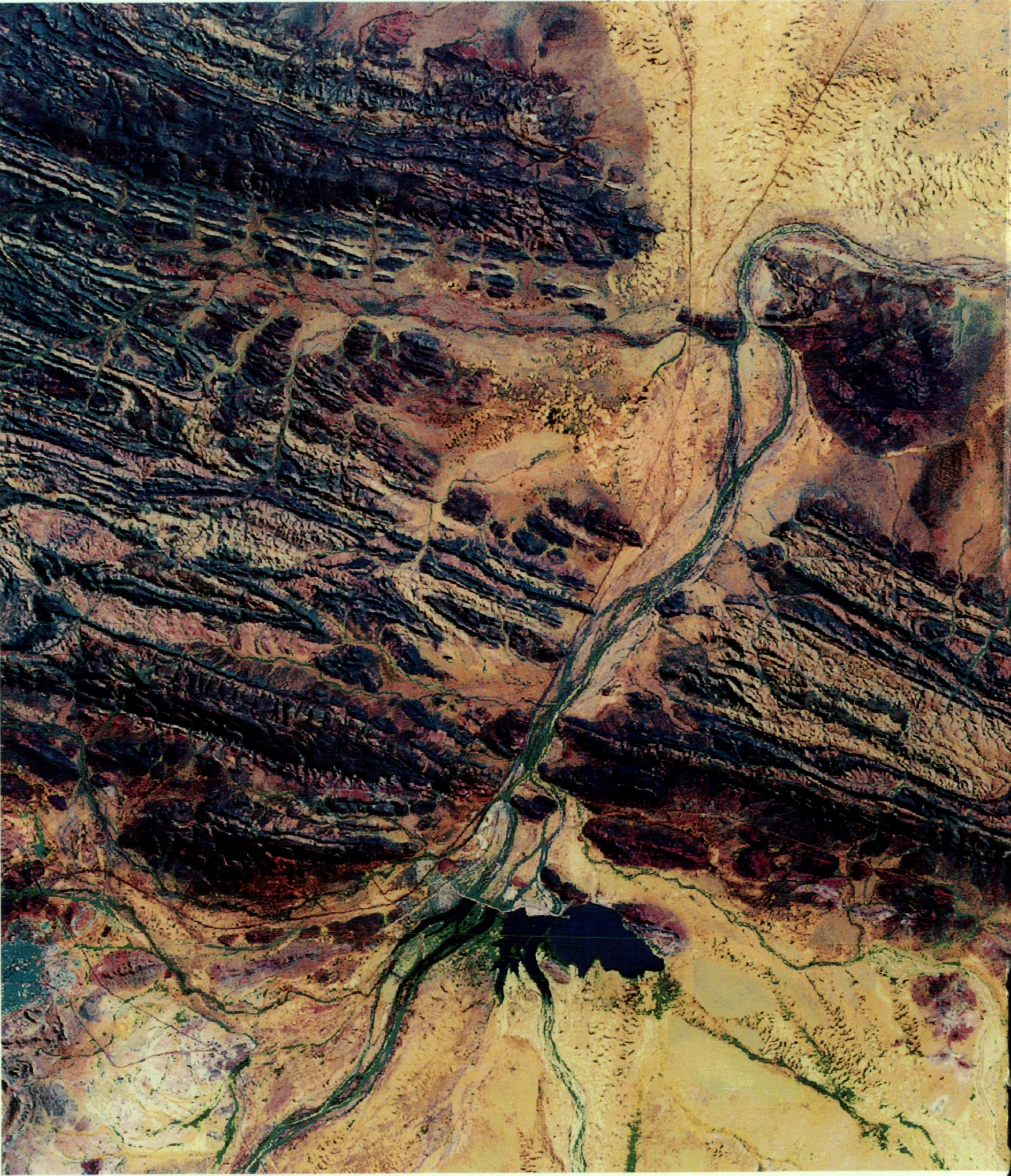

